\title{
Magnetic Resonance Elastography Using a Single-Sided \\ Constant Gradient Magnet
}

\author{
By \\ Carl Sherwin Tan \\ B.A.Sc, University of British Columbia, 2009 \\ A thesis submitted to the Faculty of Graduate and Postdoctoral Affairs \\ in partial fulfillment of the requirements for the degree of \\ Master of Applied Science in Biomedical Engineering \\ Ottawa-Carleton Institute for Biomedical Engineering (OCIBME) \\ Department of Systems and Computer Engineering \\ Carleton University \\ Ottawa, Ontario, Canada, K1S 5B6 \\ April 2011 \\ (C) Copyright 2011, Carl Sherwin Tan
}


Library and Archives

Canada

Published Heritage

Branch

395 Wellington Street

Ottawa ON K1A ON4

Canada
Bibliotheque et

Archives Canada

Direction du

Patrimoine de l'édition

395 , rue Wellington

Ottawa ON K1A ON4

Canada

Your file Votre référence

ISBN: 978-0-494-81708-7

Ourfile Notre référence

ISBN: 978-0-494-81708-7

NOTICE:

The author has granted a nonexclusive license allowing Library and Archives Canada to reproduce, publish, archive, preserve, conserve, communicate to the public by telecommunication or on the Internet, loan, distribute and sell theses worldwide, for commercial or noncommercial purposes, in microform, paper, electronic and/or any other formats.

The author retains copyright ownership and moral rights in this thesis. Neither the thesis nor substantial extracts from it may be printed or otherwise reproduced without the author's permission.
AVIS:

L'auteur a accordé une licence non exclusive permettant à la Bibliothèque et Archives Canada de reproduire, publier, archiver, sauvegarder, conserver, transmettre au public par télécommunication ou par l'Internet, prêter, distribuer et vendre des thèses partout dans le monde, à des fins commerciales ou autres, sur support microforme, papier, électronique et/ou autres formats.

L'auteur conserve la propriété du droit d'auteur et des droits moraux qui protège cette thèse. Ni la thèse ni des extraits substantiels de celle-ci ne doivent être imprimés ou autrement reproduits sans son autorisation.
In compliance with the Canadian Privacy Act some supporting forms may have been removed from this thesis.

While these forms may be included in the document page count, their removal does not represent any loss of content from the thesis.
Conformément à la loi canadienne sur la protection de la vie privée, quelques formulaires secondaires ont été enlevés de cette thèse.

Bien que ces formulaires aient inclus dans la pagination, il n'y aura aucun contenu manquant.

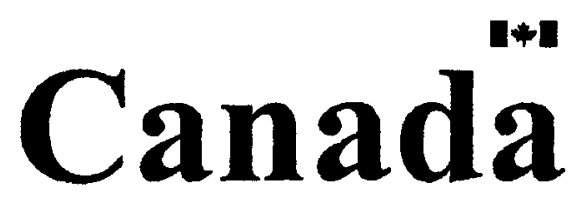


The undersigned recommend to the Faculty of Graduate and Postdoctoral Affairs acceptance of the thesis

Magnetic Resonance Elastography Using a Single-Sided Constant Gradient Magnet

Submitted by

Carl Sherwin Tan, B.A.Sc.

in partial fulfillment of the requirements for the degree of

Master of Applied Science in Biomedical Engineering

A. Marble, Thesis Co-Supervisor

Y. Ono, Thesis Co-Supervisor

H. Schwartz

Chair, Department of Systems and Computer Engineering

Carleton University

April 2011 


\begin{abstract}
Magnetic Resonance Elastography is a known technique used to measure tissue elasticity in-vivo but using traditional clinical Magnetic Resonance Imaging machines. In an attempt to greatly reduce cost of performing elastography and increase portability, this thesis investigates the potential use of a portable single-sided magnet, which is a much cheaper and more convenient alternative to conventional superconducting magnets for use in Magnetic Resonance Elastography. In this contribution, a novel velocity imaging method was designed and developed to perform elastography using a single-sided magnet. An experimental setup was then created to simulate and verify this methodology. The overall system was then evaluated in terms of being able to distinguish the stiffness of one sample from another. The experimental results suggest the potential of the developed methodology as a means of determining elasticity of materials using a single-sided constant gradient magnet.
\end{abstract}




\section{Acknowledgements}

I would like to thank my supervisors Dr. Andrew E. Marble, Assistant Professor of Systems and Computer Engineering, Carleton University and Dr. Yuu Ono, Associate Professor of Systems and Computer Engineering, Carleton University for their time, guidance and support they have provided me throughout my graduate studies. I would also like to express my appreciation to Carleton University and the Natural Sciences and Engineering Research Council of Canada (NSERC) for the opportunity and the financial support that they provided for this research and to Shari Levac (Department of Chemistry) and to Kevin Sangster and Alex Proctor (Department of Mechanical Engineering) for their technical assistance. Finally, a special thanks to my friends, and family for their enthusiasm, encouragement, patience and support. 


\section{Table of Contents}

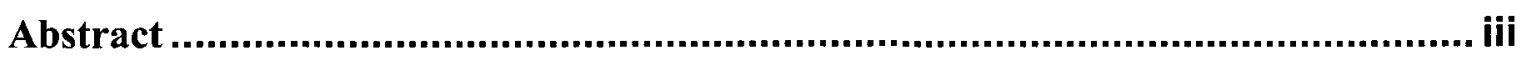

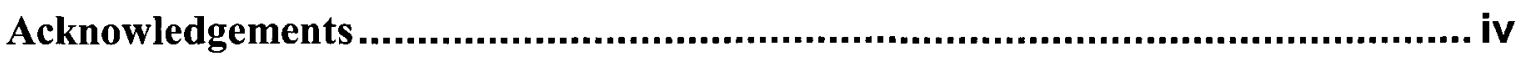

Table of Contents ...............................................................................................

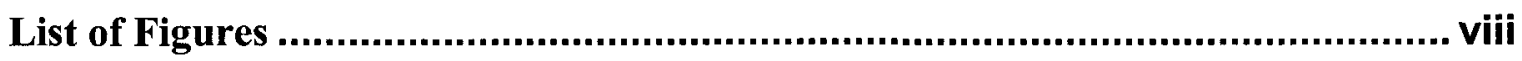

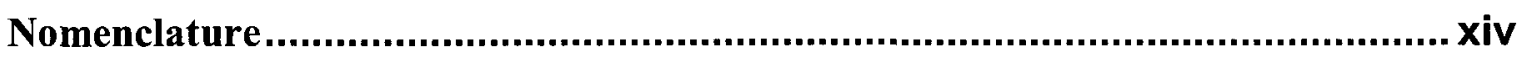

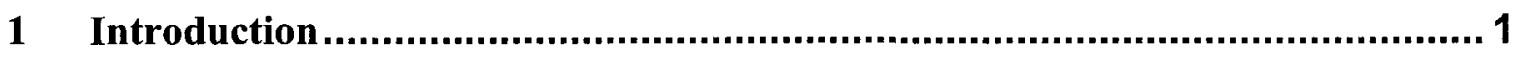

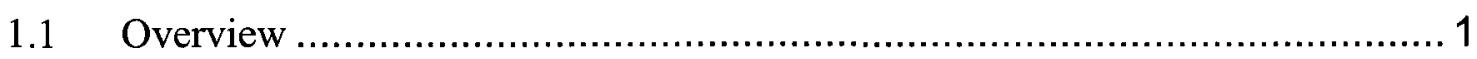

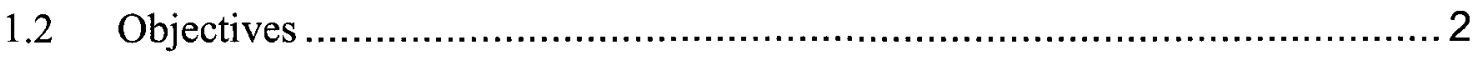

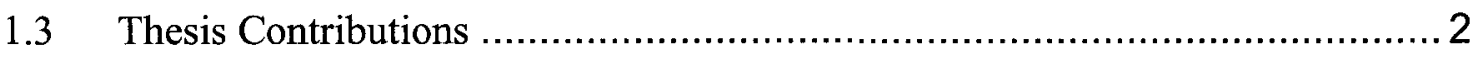

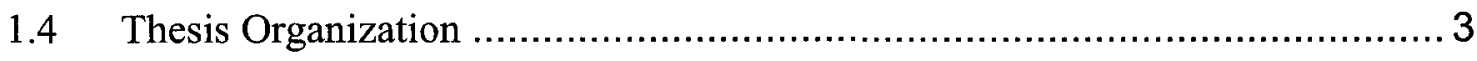

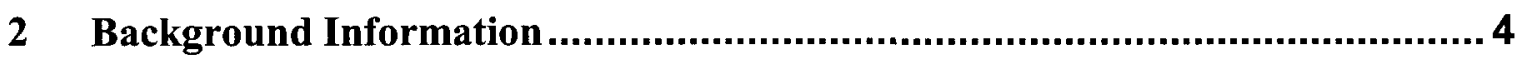

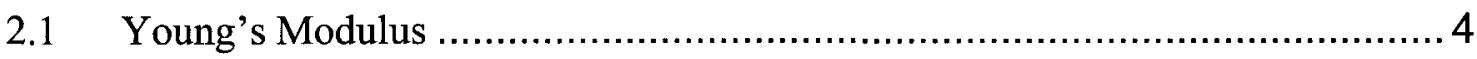

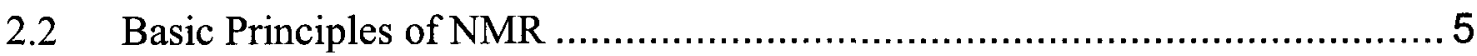

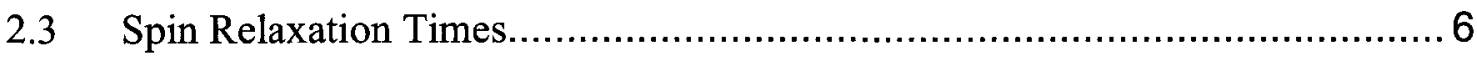

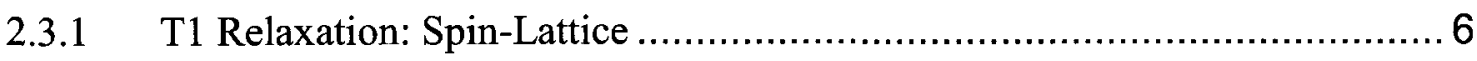

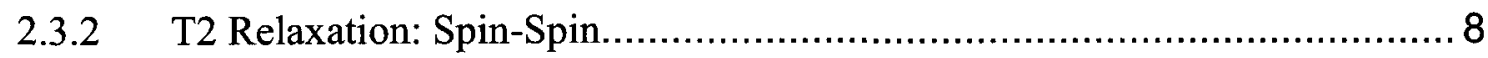

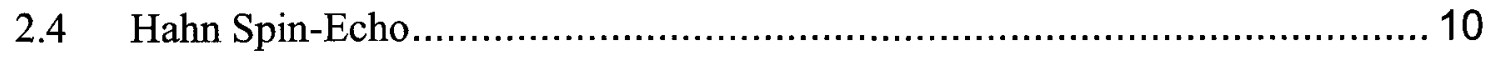

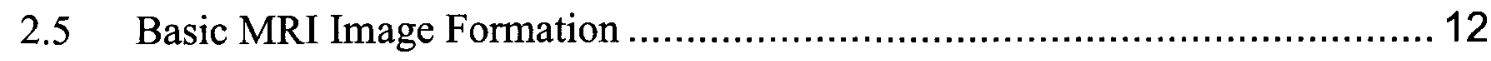

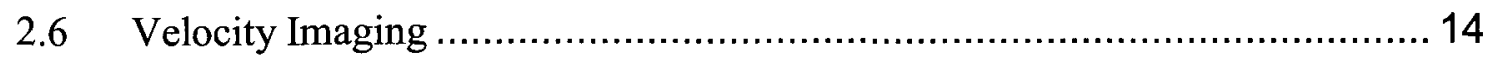

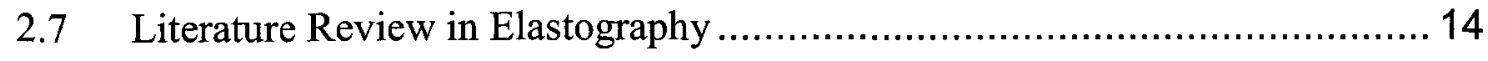


2.7.1 Ultrasound Elastography ......................................................... 15

2.7.2 Magnetic Resonance Elastography ........................................... 15

2.7.2.1 Shear Wave Generation in Tissue ............................................... 16

2.7.2.2 Wave Imaging - MRE Pulse Sequences .................................. 18

2.7.3 Elastography Comparison and Improvements ................................. 20

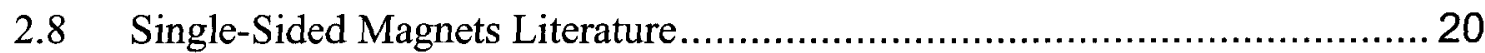

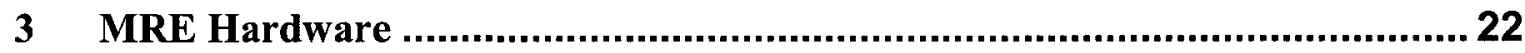

3.1 MRI Pulse Synthesizer Hardware Specifications .................................. 23

3.1.1 Bruker Optics NMR Scanning Parameters ....................................... 23

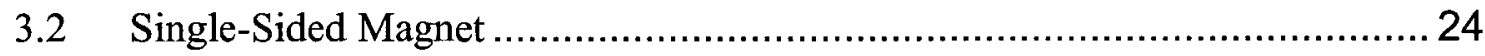

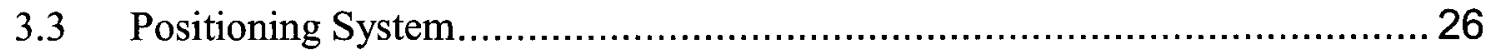

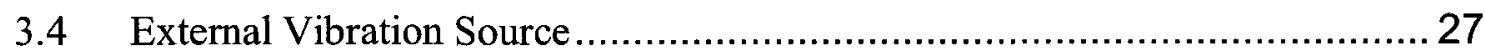

4 Velocity Measurements and Pulse Sequences .............................................. 31

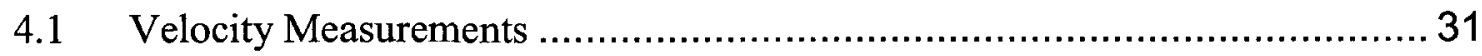

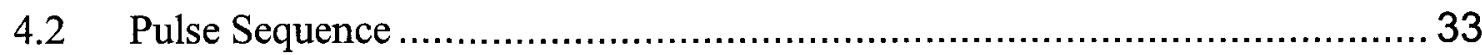

4.3 Data Processing Matlab Velocity Measurements ...................................... 35

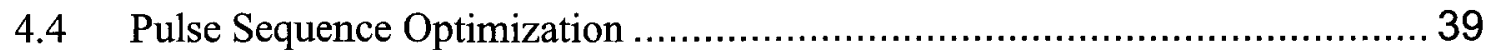

5 Simulation Environments and Experiments ......................................... 41

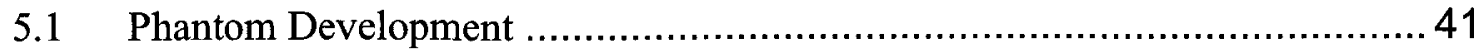

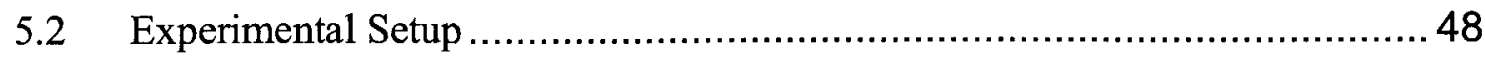


5.2.1 Optimizing Repetition Time

5.2.2 Massager Placement

5.2.3 Preliminary Motion Experiments - Conair Massager

53

5.2.3.1 Preliminary MRI Measurements and Results ................................ 54

5.2.3.2 Preliminary Measurements - Discussion ......................................59

5.2.3.3 Preliminary Measurements - Acquisition Time Optimization ............ 60

5.2.3.4 P-space Measurements and Results ........................................... 61

5.2.4 Motion Experiments with Modified Setup - Dr. Scholl's Massager ........ 66

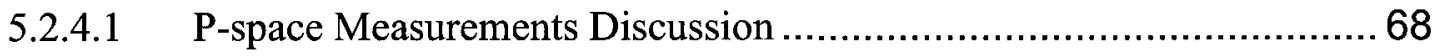

5.2.4.2 Ethylene Glycol Gelatin Elasticity Measurements and Results ............70

5.2.4.3 Ethylene Glycol Gelatin Elasticity Discussion .............................. 75

6 Conclusion and Future Work .................................................................. 77

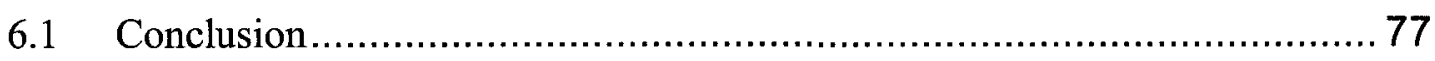

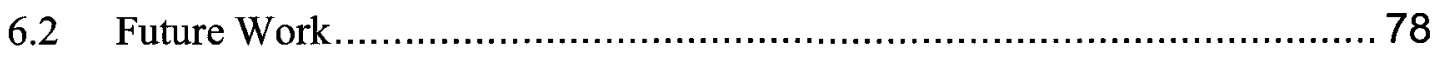

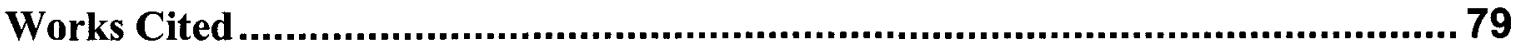




\section{List of Figures}

Figure $1-\mathrm{T} 1$ relaxation curve where $\mathrm{T} 1$ is $1 \mathrm{~s}$ and $\mathrm{RF}$ pulse is applied at 0 s. Note that at $1 \mathrm{~s}, \mathrm{M}_{\mathrm{z}}$ has recovered approximate $63 \%$ of the original signal and at $5 \mathrm{~s}$ or $5 * \mathrm{~T} 1$, the signal is $99 \%$ recovered. Therefore $\boldsymbol{\tau}_{\mathrm{r}}$ is usually set at least at $5^{*} \mathrm{~T} 1$. 7

Figure 2 -a) A microscopic illustration of the transverse magnetization vector for each spin after a $90^{\circ}$ pulse. It shows that after some time $t$, spins go out of phase with each other due to energy transfer. b) A macroscopic illustration of the net transverse magnetization showing that after a $90^{\circ}$ pulse, $\mathrm{M}_{\mathrm{xy}}$ decays due to phase differences

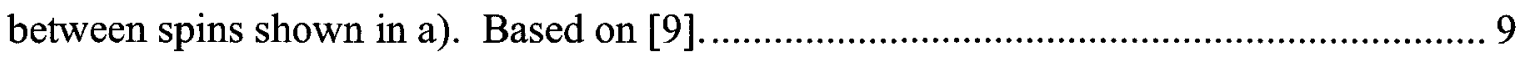

Figure $3-\mathrm{T} 2$ relaxation curve where $\mathrm{T} 2$ is $1.5 \mathrm{~s}, \mathrm{~T} 2$ ' is $0.6 \mathrm{~s}$. RF pulse is applied at $0 \mathrm{~s}$. Note that $\mathrm{T}^{*}$ is much shorter than $\mathrm{T} 2$ due to the effect of $\mathrm{T} 2$ '. Typically, a CPMG or Spin-Echo is used to observe T2 decay. All data should be acquired before $3^{*} \mathrm{~T} 2$ has elapsed to have a reasonable signal-to-noise ratio _...................................................... 10

Figure $4-$ A typical CPMG Pulse sequence diagram where $\tau$ is the time between pulses

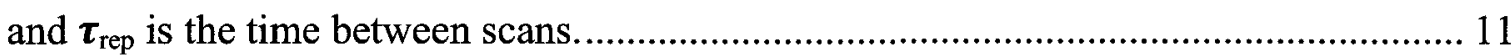

Figure 5 - The peaks of the echoes that are formed after the $180^{\circ}$ refocusing pulse obeys T2 decay 12

Figure 6 - Matlab generated sliced image phantom and its corresponding k-space frequency map. The center of $\mathrm{k}$ space shows the low spatial frequency component. ..... 13

Figure 7 - A block diagram of the experimental setup used in this thesis. 22

Figure 8 - Bruker Minispec System. The preamplifier is the small box on the left and the $\mathrm{RF}$ pulse generator is shown on the right 23 
Figure 9 - A Schematic Diagram of the single-sided magnet used for this work. The shaded region is the "sweet spot" of the magnet where there is a relatively constant magnetic field gradient $B_{1}$. Reproduced from [72] ............................................... 25

Figure 10 - Single-sided magnet used for this work. ................................................. 26

Figure 11 - The Velmex position system as setup in the lab.......................................... 27

Figure 12 - Conair Massager with the labels L (large frequency) and S (small frequency) to identify the two different frequencies it can generate. 28

Figure 13 - Output show on a Tektronix Digital Oscilloscope from piezoelectric sensor when the " $L$ " side of the Conair Massager is applied. We can see that the vibration frequency is approximately $59 \mathrm{~Hz}$. 28

Figure 14 - The image above is the Dr. Scholl's Hand-held Massager (Model DR7565). For the work done in this thesis, we used the top surface of the massager as the vibrating source. 29

Figure 16 - SGSE-CPMG pulse sequence. The SGSE is the part of the pulse sequence that is sensitive to motion. The CPMG Echo Train is there to improve signal-to-noise ratio and to effectively induce an alternating gradient field on the sample. For our work, $\theta$ is $90^{\circ}$. 34

Figure 17 - A CPMG echo train with a static gradient effectively produce an oscillating gradient due to the $180^{\circ}$ pulse flipping the direction of the spins. Flipping the rotation of the spins with a $180^{\circ}$ pulse is the same as applying a negative gradient on the spins. .... 35 Figure 18 - Raw signal of two echoes obtained using the Bruker system on a $0.225 \mathrm{mg} / \mathrm{L}$ gelatin sample where red is the real data and blue is the imaginary data. 36 Figure 19 - A Typical MRI (SGSE-CPMG) scan of a $0.25 \mathrm{~g} / \mathrm{mL}$ ethylene glycol gelatin 
sample obtained using the Bruker system. The scanning parameters were: 400 Scans, 80 Points per Echo, $0.1 \mathrm{~ms}$ Acquisition time, $\boldsymbol{\tau}=0.2 \mathrm{~ms}, \boldsymbol{\tau}_{\text {train }}=0.2 \mathrm{~ms}$ and 601 Echoes. Red is the real data and yellow is the imaginary data. 36

Figure 20- To the top shows raw MRI signal with 10 echoes. Summing all the echoes in the same time sequence will result in an echo shown in the bottom image. To complete ensemble averaging, the figure on the bottom would need to have its signal intensity divided by the number of echoes (10). The $\mathrm{x}$-axis of the bottom figure represents the number of data points in each echo that were sampled by the Bruker, the Minispec. In this case, each echo contains 80 points. 38

Figure 21 - Obtained peaks of echo train for a $0.3 \mathrm{~g} / \mathrm{mL}$ Gelatin Sample with and without the pulse spacing correction. It is observed that with the timing correction, the signal echoes have a higher signal intensity than with no correction. The scanning parameters were: 240 Scans/point, 80 Points per Echo, 0.1 Acquisition time, and 100 Echoes. 40

Figure 22 - Three of the five ethylene glycol gelatin samples created for this experiment.

Figure 23 - A Block Diagram for the setup in measuring Young's modulus 45 Figure 24 - VXM step motor and display. The display shows the displacement on any axis in $\mathrm{mm}$ or in inches. 45

Figure 25 - Gram scale with an ethylene glycol sample. A small cylindrical rod is mounted underneath the arm and is slightly pressing on the sample. The scale is set to zero at this position. 46

Figure 26 - Displacement vs Force graph for the created gelatin samples. 46 Figure 27 - Young's Modulus vs Gelatin Concentration plot with an error bar of $+/-1$ 
standard deviation (eq 5.3). It shows that with increasing gelatin concentration, the Young's Modulus also increases. The calculated Young's modulus for the created samples are $276,365,434,457,559 \mathrm{kPa}$.

Figure 28 - Average Signal Intensity at each TR. We can observe that at approximately $1000 \mathrm{~ms}$, the signal intensity does not significantly improve anymore. The scanning parameters were: 400 Scans/point, 80 Points per Echo, 0.3 Acquisition time, and 101 Echoes. 49

Figure 29 - Signal Intensity vs Massager Distance from the Surface of the Magnet. The massager used for this data is the Conair Massager. We can see that when the massager is close to the magnet, the signal intensity is much lower than when the massager is placed farther away from the magnet. The scanning parameters were: 1000 Scans/point, 80 Points per Echo, 0.3 Acquisition time, and 200 Echoes. 51 Figure 30 - Signal Intensity vs Massager Distance from the Surface of the Magnet. The massager used for this data is the Dr. Scholl's Massager. The scanning parameters were: 128 Scans/point, 80 Points per Echo, 0.1 Acquisition time, and 300 Echoes.

Figure 31 - Slice View block diagram of the first experimental setup for preliminary experiments. 53

Figure 32 - Experiment setup with Conair Massager. 54

Figure 33 - Measured signal Intensity of silicon rubber under no motion at various $\tau$. T2 is measured from 0 until $200 \mathrm{~ms}$ or until the signal-to-noise ratio is too low. 55

Figure 34 - Measured signal Intensity for silicon rubber under motion at various $\boldsymbol{\tau}=0.2$, $0.3,0.4,0.5$. T2 is measured from 0 until $200 \mathrm{~ms}$ or until the signal-to-noise ratio is too low 56 
Figure 35 - Measured signal Intensity for silicon rubber under motion at various $\boldsymbol{\tau}$. T2 is measured from 0 until $200 \mathrm{~ms}$ or until the signal-to-noise ratio is too low.

Figure $36-\mathrm{T} 2$ vs $\tau$ plot sample under no motion. It shows that $\mathrm{T} 2$ does not change statistically as $\tau$ increases. 58

Figure $37-\mathrm{T} 2$ vs $\boldsymbol{\tau}$ plot sample under motion. It shows that $\mathrm{T} 2$ starts to decrease at $\boldsymbol{\tau}$ greater than $0.4 \mathrm{~ms}$. At $\tau>1 \mathrm{~ms}$, the remaining signal is mostly noise. 58

Figure 38 - Plot of 10 echoes with varying $a c q$ at 480 scans on a $0.225 \mathrm{~g} / \mathrm{mL}$ ethylene glycol sample. 61

Figure 39 - Resulting P-space plot on silicon rubber with no applied motion. The velocity distribution curve resembles a delta function centered at 0 velocity.

Figure 40 - Resulting P-space plot for Silicon Rubber with Applied Motion. The velocity distribution curve has a shaped distribution implying motion is measured.........63

Figure 41 - Resulting P-space plot for Ethylene Glycol with no Applied Motion. The velocity distribution curve resembles a delta function centered at 0 velocity. 64

Figure 42 - Resulting P-space plot for Silicon Rubber with Applied Motion. The velocity distribution curve has a bell shape distribution implying that motion is measured.

Figure 43 - Wooden Platform that is mounted on top of the magnet array...................... 66

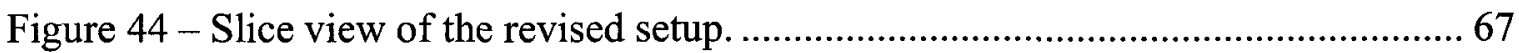

Figure 45 - Experiment setup with Dr. Scholl's Massager. ........................................... 68

Figure $46-\tau 2$ from 0.02 to 2.02 with an increment of $0.04,400$ scans, $0.1 \mathrm{~ms}$ acquisition time, 301 echoes and at 80 data points per echo

Figure 47 - Echo Peaks of Raw MRI data at various $\tau$ for $0.225 \mathrm{~g} / \mathrm{mL}$ gelatin under 
motion.

Figure 48 - P-Space plot for all the 5 ethylene glycol gelatin samples under motion. Two plots are shown with no vibration.

Figure 49 - Velocity Probability Distribution of the five gelatin samples. A close up on the center distribution is shown in Figure 48 73

Figure 50 - Velocity Probability Distribution focused at the center. 73

Figure 51 -RMS Velocity vs Gelatin Concentration....................................................... 74

Figure 52 -RMS Velocity vs Estimated Young's modulus................................................ 74 


\section{Nomenclature}

\section{List of Abbreviations}

\begin{tabular}{|c|c|}
\hline Abbreviations & Descriptions \\
\hline CPMG & Carr-Purcell-Meiboom-Gill sequence \\
\hline CWI & Compliance-Weighted Imaging \\
\hline DENSE & Displacement Encoding with Stimulated Echoes \\
\hline FID & Free Induction Decay \\
\hline$\overline{\text { FSE }}$ & Fast Spin Echo \\
\hline ME-PC-GE & Mutli-Echo Phase Contrast Gradient-Echo sequence \\
\hline MEG & Motion Encoding Gradient \\
\hline MRE & Magnetic Resonance Elastography \\
\hline MRI & Magnetic Resonance Imaging \\
\hline NMR & Nuclear Magnetic Resonance \\
\hline $\mathrm{OCE}$ & Optical Coherent Elastography \\
\hline $\mathrm{RF}$ & Radio Frequency \\
\hline RMS & Root Mean Square \\
\hline SGSE & Static Gradient Spin Echo \\
\hline TE & Echo Time \\
\hline TR & Repetition Time \\
\hline UE & Ultrasound Elastography \\
\hline
\end{tabular}




\section{List of Symbols}

\begin{tabular}{|c|c|c|}
\hline Symbol & Description & Units \\
\hline$a c q$ & The time interval in which MRI data is acquired & $\mathrm{ms}$ \\
\hline Arb. Unit & Arbitrary Units & \\
\hline$A_{0}$ & Original cross-section area through which the force is applied & $\mathrm{m}^{2}$ \\
\hline $\mathrm{B}_{\mathrm{o}}$ & Magnetic Field Strength & $\mathrm{T}$ \\
\hline$E$ & Young's Modulus & $\mathrm{kPa}$ \\
\hline$\overline{E_{\text {pulse }}}$ & Energy of the radio frequency pulse & $\mathbf{J}$ \\
\hline$F$ & Force applied & $\mathrm{N}$ \\
\hline$G$ & First spatial derivative of the magnetic field & $\mathrm{T} / \mathrm{m}$ \\
\hline$k$ & Wave Vector & $1 / \mathrm{m}$ \\
\hline$\Delta L$ & Amount of deformation experienced by the sample. & $\mathrm{m}$ \\
\hline$L_{0}$ & Initial length of the sample. & $\mathrm{m}$ \\
\hline $\mathrm{M}_{\mathrm{xy}}$ & Signal Magnitude in the transverse magnetization plane & \\
\hline $\mathrm{M}_{\mathrm{z}}$ & Signal Magnitude parallel to the direction of the magnetic field. & \\
\hline$p$ & Reciprocal Velocity Image Space & $\mathrm{s} / \mathrm{m}$ \\
\hline$r$ & Position vector of the location of a particle & $\mathrm{m}$ \\
\hline$S$ & Signal Intensity detected by the MRI system. & \\
\hline$t_{90}$ & Duration of the $90^{\circ}$ pulse. & $\mathbf{s}$ \\
\hline$T$ & Period of the induced mechanical excitation and oscillating gradient & $\mathrm{s}$ \\
\hline $\mathrm{T} 1$ & Spin-lattice relaxation time & $\mathrm{s}$ \\
\hline $\mathrm{T} 2$ & Transverse relaxation time & $\mathrm{s}$ \\
\hline T2' & Relaxation process due to the in-homogeneous magnetic field & $\mathrm{s}$ \\
\hline
\end{tabular}




\begin{tabular}{|ccc|}
\hline T2* & Observed relaxation process due to T2' and T2 & $\mathbf{s}$ \\
\hline $\boldsymbol{\varepsilon}_{o}$ & Vector of the mechanical displacement amplitude & \\
\hline$\tau$ & Time between $90^{\circ}$ and $180^{\circ}$ pulses & $\mathrm{s}$ \\
\hline$\tau_{\text {rep }}$ & Time between successive $180^{\circ}$ pulses & $\mathrm{s}$ \\
\hline$\omega$ & Larmor Frequency & $\mathrm{rad} / \mathrm{s}$ \\
\hline$\gamma$ & Gyromagnetic ratio & $\mathrm{rad} / \mathrm{s} / \mathrm{T}$ \\
\hline
\end{tabular}




\section{Introduction}

This chapter will provide an overview of the whole document and its organization. The purpose, objective, and contributions of this research work will be discussed.

\subsection{Overview}

Medical science over the years has found many ways in diagnosing and preventing cancer. Current mainstream treatment options are limited to chemotherapy, radiation therapy and surgery [1]. The success rate of these current treatments is primarily based on the current stage of the cancer. Stage 1 is when cancer is localized to one part of the body and is highly curable, while Stage 4 is when cancer metastasized or spread to other areas of the body which can cause death. Being able to detect cancer at an earlier stage can vastly increase the chance of the cancer patient being treated using currently available treatment options. Palpation is a technique that is used by doctors that can detect early signs of cancers by "feeling" the stiffness of tissues on the body. Cancer tissue are typically 5-20 times stiffer compared to normal tissue [2]. Therefore, abnormal stiffness of tissue can indicate potential cancer or tumors. However, this technique is limited to tissues near the surface of the body. To find tissue stiffness in-vivo, Magnetic Resonance Elastography (MRE) can be used. MRE images the stiffness of tissue, providing similarly useful information as in palpation. The drawback of MRE is the cost and accessibility of the system as it requires large expensive medical MRI systems which 
hinders overall global availability and usage of the technology. The motivation of our research is to reduce the overall cost of performing MRE to improve access to this valuable early detection technique. By using existing low field single-sided magnets to perform elastography measurements, the cost could be greatly reduced for these systems.

\subsection{Objectives}

This research focuses on using a single-sided magnet to perform MRE measurements in an attempt to significantly reduce cost and increase portability. Currently, no published literature has studied the possibility of such a system. This thesis strives to develop novel methods in terms of setup, pulse sequence and data processing to verify such system is possible to implement.

\subsection{Thesis Contributions}

The following list will highlight the main contributions that are presented thoroughly in this thesis:

- Designed and implemented a novel MRE system for determining material elasticity using a single-sided magnet. An experimental setup was created to test and validate the system.

- Implemented pulse sequences that are used to perform velocity encoding in determining the elasticity of the samples. 
- Developed tissue mimicking phantom gelatin gels with varying stiffness to evaluate the single-sided MRE system in a controlled environment.

- Developed and implemented an algorithm to process raw MRI data for the purpose of obtaining elasticity measurements.

\subsection{Thesis Organization}

The thesis is broken down into six chapters. Chapter 1 introduces the purpose, objective and organization of the thesis. Chapter 2 provides background information on theory and concepts related to the work performed. In addition, discussion of current elastography techniques is also provided. Chapter 3 describes the hardware and equipment used in the experiments. Chapter 4 discusses the theories and ideas developed for this thesis. Chapter 5 details the experiments and work conducted in verifying the plausibility of performing MRE using a single-sided magnet. Chapter 6 concludes the thesis by summarizing major findings and discussing about future work. 


\section{Background Information}

In this section, concepts necessary to understand the procedures and theories used in the thesis work is introduced. Furthermore, this section will provide a review of existing literature relating to elastography.

\subsection{Young's Modulus}

Elasticity is a material property that quantifies a material's ability to return to its original shape after an applied force is removed. Elasticity can be characterized by the Young's Modulus given by [3]

$$
E=\frac{F L_{0}}{A_{0} \Delta L}
$$

where $E$ is Young's modulus, $F$ is the force applied, $A_{0}$ original cross-section area through which the force is applied, $L_{0}$ is the original length of the object and $\Delta L$ is the amount of deformation. Applying a constant amplitude oscillating force and then taking the derivative of Eq. 2.1, we can see that the displacement velocity is inversely proportional to the material's Young's Modulus.

$$
\begin{aligned}
& \Delta L=\frac{F \cos \left(\omega_{0} t\right) L_{0}}{A_{0} E} \\
& \frac{d L}{d t}=-\frac{F \omega_{0} \sin \left(\omega_{0} t\right) L_{0}}{A_{0} E}
\end{aligned}
$$

This provides the basis of the idea that a stiff material or a material with a high Young's Modulus will experience a smaller displacement velocity than a soft material or smaller Young's Modulus assuming a constant oscillating force is applied and the samples to be 
tested are similar in size.

\subsection{Basic Principles of NMR}

MRI was discovered in the 1970's by Paul C Lauterbur and Sir Peter Mansfield when they saw that the nuclear magnetic resonance (NMR) phenomenon could be used to produce images in-vivo of the human body [4]. The basis of NMR is that all stable isotopes with an odd number of protons and/or neutrons have a non-zero spin [5]. This means that the isotpes has an intrinsic magnetic moment and angular momentum. When a sample experiences a magnetic field $B_{0}$, the isotopes will align parallel to it (lower energy state) or anti parallel (higher energy state) resulting in a small net dipole moment in the direction of the magnetic field [4] [5]. These dipoles will "wobble" around the direction of the magnetic field. This process is called precession. The frequency they precess at is called the Larmor Frequency given by [6]

$$
\omega(\boldsymbol{r})=\gamma \mathrm{B}_{0}+\gamma G \cdot \boldsymbol{r}
$$

where $\omega$ is the Larmor Frequency, $G$ is the first spatial derivative of the field, $B_{0}$ is the magnitude of the static gradient field, $\gamma$ is the gyromagnetic ratio and $r$ is the spin coordinates. At equilibrium, the transverse magnetic component $M_{x y}$ points in all direction and cancels out. The magnetic component $\mathrm{M}_{\mathrm{z}}$, parallel to $\mathrm{B}_{\mathrm{o}}$, is equal to the net magnetization vector.

To generate an MRI signal a radio frequency (RF) pulse is applied. To rotate the net magnetization vector by $90^{\circ}$, the RF pulse must have an energy $E_{\text {pulse }}=\hbar \omega$ where $\omega$ is $2 \pi$ multiplied by the Larmor frequency and $\hbar$ is the reduced planck constant. To achieve 
other flip angles $\alpha$, the energy level of the RF pulse can be varied. This pulse excites the dipoles causing $M_{z}$ to decrease and causes the dipoles to precess in phase causing $M_{x y}$ to increase. After the RF pulse is turned off, the spins will start to realign and return to their original state causing $M_{z}$ to increase and $M_{x y}$ to decrease. The rate at which $M_{x y}$ decreases is called transverse relaxation time (T2) and the rate at which $\mathrm{M}_{\mathrm{z}}$ re-establish itself is called spin-lattice relaxation time (T1) [7] [8]. The relaxation times are a statistical average for an ensemble of spins [5]. RF energy is released back as the dipoles go from a higher energy state to a lower energy state and RF coils detect this energy. The resulting signal observed is referred to as the free induction decay (FID) response signal. In medical imaging, the isotope that is commonly used is the hydrogen atom (protons) as it is the most abundant in the human body, where its gyromagnetic ratio $\gamma$ is $2 \pi^{*} 42.58$ $\mathrm{MHz} / \mathrm{T}$.

\subsection{Spin Relaxation Times}

The process in which the spins realign after an RF pulse is called spin relaxation. There are two main types of relaxation, $\mathrm{T} 1$ relaxation, and $\mathrm{T} 2$ relaxation. Relaxation time is a statistical average for an ensemble of spins [5].

\subsubsection{T1 Relaxation: Spin-Lattice}

An excitation pulse is applied to the system to push it away from equilibrium. In equilibrium, the magnetization vector is parallel to the $\mathrm{z}$ axis. Applying a $90^{\circ}$ pulse, the 
magnetization vector is toppled onto the $x-y$ plane. The process in which the spins release the energy obtained from the $R F$ pulse back to its surrounding is called spinlattice relaxation or longitudinal relaxation. This process is described by [7]

$$
M_{z}(\tau)=M_{z}(0)\left(1-e^{-\tau / T 1}\right)
$$

where $\mathrm{M}_{\mathrm{z}}$ is magnetization component in the $\mathrm{z}$-axis ,T1 is the Spin-Lattice time constant and $\tau$ is the time following the $90^{\circ}$ excitation pulse (Fig. 1). The value of $\mathrm{T} 1$ varies depending on the mobility of sample's lattice, the gyromagnetic ratio of the nucleus and on $B_{0}$. The importance of $T 1$ is that it sets the limit of the pulse repetition time ( $\tau_{\text {rep }}$ or TR) or it sets the limit on how fast a pulse sequence can be repeated.

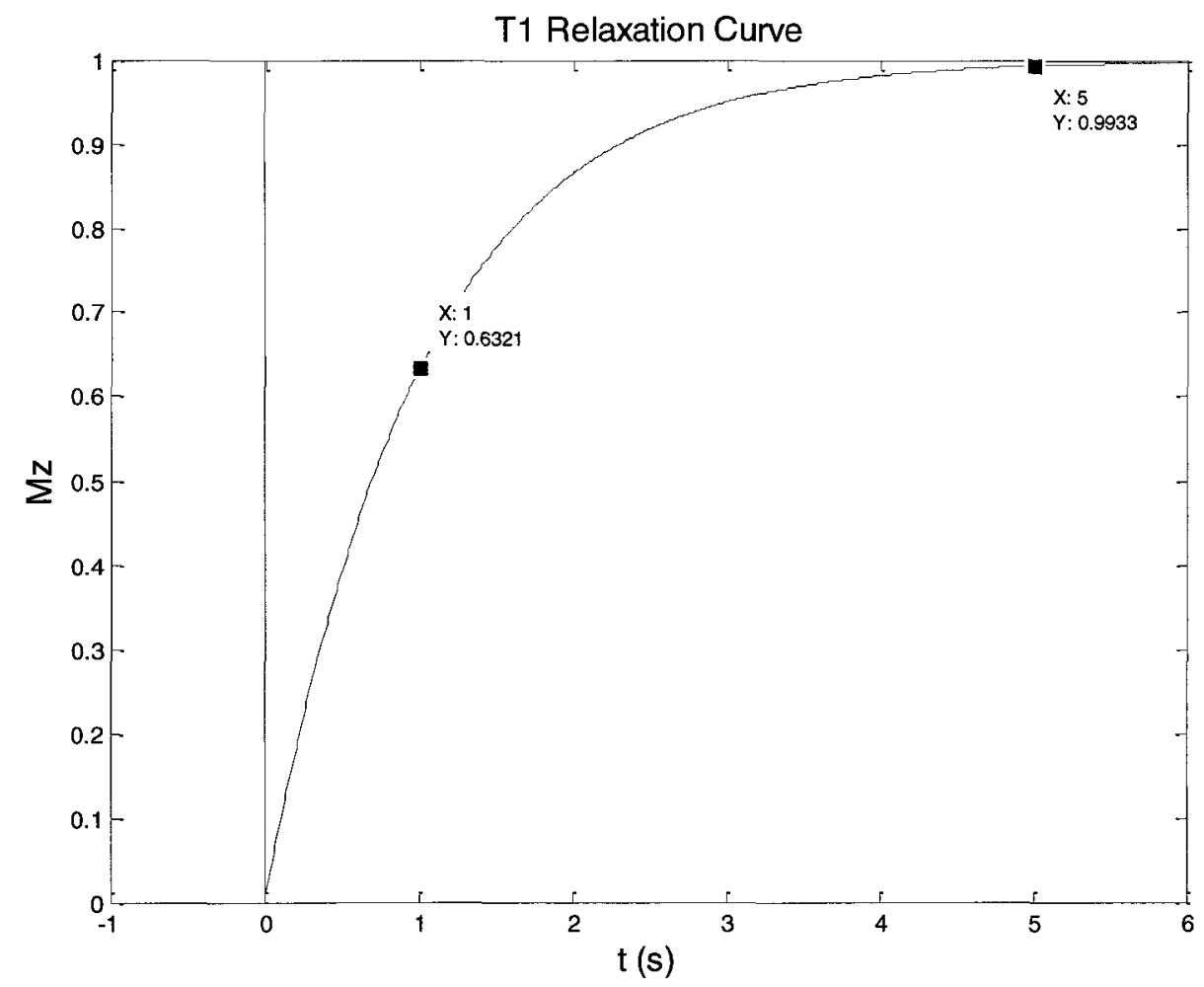

Figure 1 - T1 relaxation curve where T1 is $1 \mathrm{~s}$ and RF pulse is applied at 0s. Note that at $1 \mathrm{~s}, M_{z}$ has recovered approximate $63 \%$ of the original signal and at $5 \mathrm{~s}$ or $5^{*} \mathrm{~T} 1$, the signal is $99 \%$ recovered. Therefore $\tau_{\mathrm{r}}$ is usually set at least at $5^{*} \mathrm{~T} 1$. 


\subsubsection{T2 Relaxation: Spin-Spin}

T1 relaxation time describes the process in which energy is released from the spins back to its surroundings, T2 relaxation time describes the loss of phase coherence due to spin-spin interaction. After a $90^{\circ} \mathrm{RF}$ pulse is applied, the transverse magnetic field $\mathrm{M}_{\mathrm{xy}}$ will be at its maximum value. As time passes, the phase between spins vectors will gradually loose coherence due to local variations in the magnetic field and to energy transfer between spins resulting in a reduced $\mathrm{M}_{\mathrm{xy}}$ (Fig. 2). To take into consideration the phase coherence due to in-homogeneous magnetic field $\mathrm{B}_{\mathrm{o}}$, a relaxation time $\mathrm{T} 2{ }^{*}$ can be defined as [4]

$$
\frac{1}{T 2^{*}}=\frac{1}{T 2}+\frac{1}{T 2^{\prime}}
$$

where $\mathrm{T} 2$ is the relaxation process for the spin-spin process and $\mathrm{T} 2{ }^{\prime}$ is the relaxation process due to the in-homogeneous magnetic field. The $\mathrm{T} 2$ relaxation process can then be described by [6]

$$
M_{x y}(\tau)=M_{x y}(0) e^{-\tau / T 2^{*}}
$$

where $M_{x y}$ is the magnetization vector in the $\mathrm{x}$-y plane (Fig. 3). Typical values for T2 are equal to or less than T1. An important point to note is that $\mathrm{T}^{*}$ is the decay that will be observed after a $90^{\circ}$ pulse is applied. T2 is the relaxation time that is dependent on the sample whereas $\mathrm{T}^{\prime}$ ' is dependent on the local magnetic field variance. Therefore to be able to distinguish $\mathrm{T} 2$ from $\mathrm{T} 2 *$, a more sophisticated $\mathrm{RF}$ pulse sequence can be applied by constantly refocusing the spins. The resulting signal strength will be affected only by the longer T2 signal. These common pulse sequences will be discussed in section 2.4. 

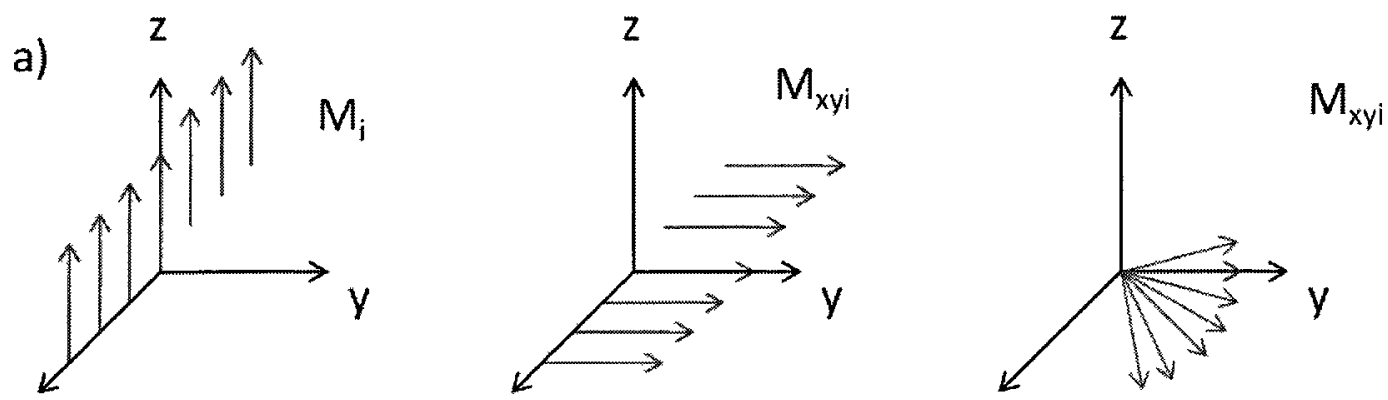

$x$

$x$

$x$

b)

$90^{\circ}$ RF Pulse
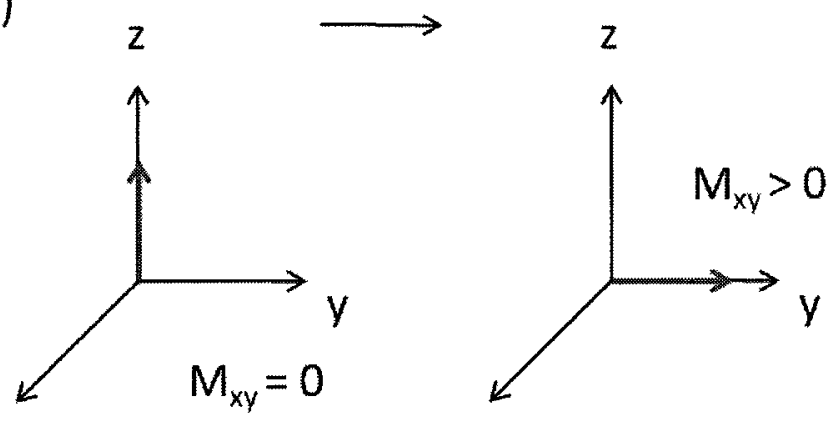

After Some Time $t$

X

$x$

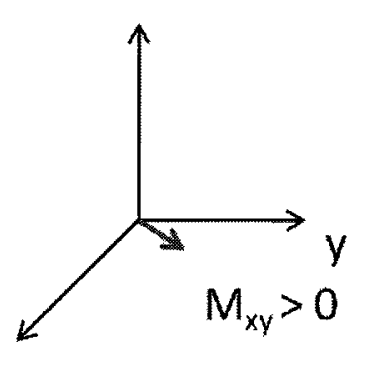

$x$

Figure 2 - a) A microscopic illustration of the transverse magnetization vector for each spin after a $90^{\circ}$ pulse. It shows that after some time $t$, spins go out of phase with each other due to energy transfer. b) A macroscopic illustration of the net transverse magnetization showing that after a $90^{\circ}$ pulse, $M_{x y}$ decays due to phase differences between spins shown in a). Based on [9]. 


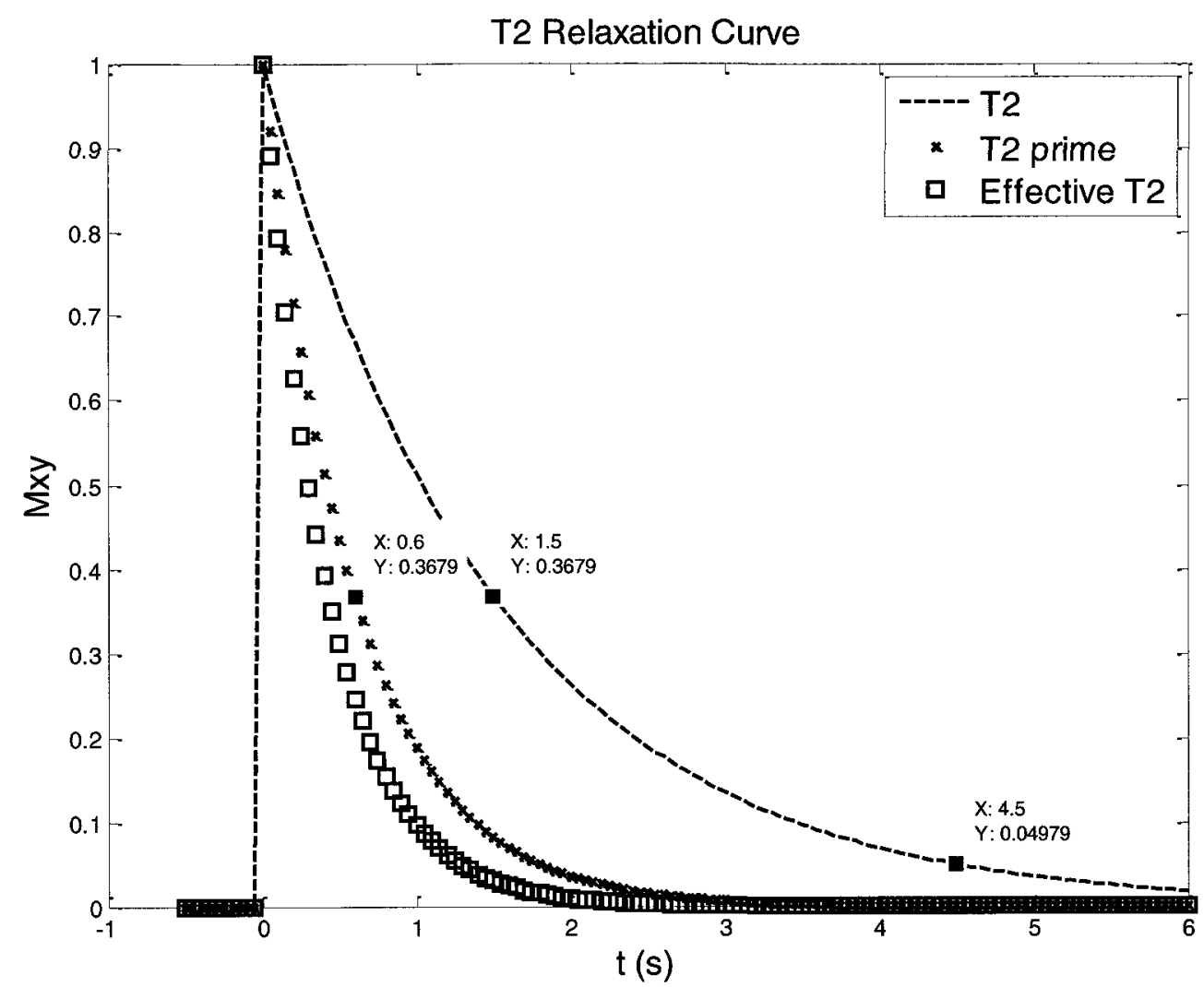

Figure $3-\mathrm{T} 2$ relaxation curve where $\mathrm{T} 2$ is $1.5 \mathrm{~s}, \mathrm{~T} 2$ ' is $0.6 \mathrm{~s}$. RF pulse is applied at 0s. Note that $T^{*}$ is much shorter than $T 2$ due to the effect of T2'. Typically, a CPMG or Spin-Echo is used to observe T2 decay. All data should be acquired before $3 * \mathrm{~T} 2$ has elapsed to have a reasonable signal-to-noise ratio.

\subsection{Hahn Spin-Echo}

After a $90^{\circ}$ pulse is applied, de-phasing of spins is caused by both spin-spin interactions and by local variations in the Larmor frequency due to the in-homogenous magnetic field. To minimize the influence of the inhomogeneous magnetic field, a Hahn Spin-Echo ( $180^{\circ} \mathrm{RF}$ pulse) can be applied to refocus the spins by reversing the phase of 
their spins. This situation can be compared to any race on a closed track. The excitation pulse $\left(90^{\circ} \mathrm{RF}\right.$ pulse) initiates the race. The variation in the local magnetic field causes some racers to be faster than other racers. After some time $\tau$, the track is inverted $\left(180^{\circ}\right.$ $\mathrm{RF}$ pulse) causing all the racers to go in reverse. At time $2 \tau$, the faster racers would have caught up to the slower racers causing an echo to form. The time it takes from the initial pulse to the peak of the echo is called the echo time (TE). The amplitude of the echoes follows T2 relaxation decay, which sets the maximum time for TE. A commonly used pulse sequence that takes advantage of spin-echo is called a Carr-Purcell-Meiboom-Gill (CPMG) sequence (Fig. 4). This sequence is commonly used to either obtain the T2 decay of a sample $[\mathbf{8}]$ or improve signal-to-ratio (SNR) of an encoding sequence $[\mathbf{1 0}]$. The modified CPMG pulse sequence developed for this work will be discussed in section 4.2.

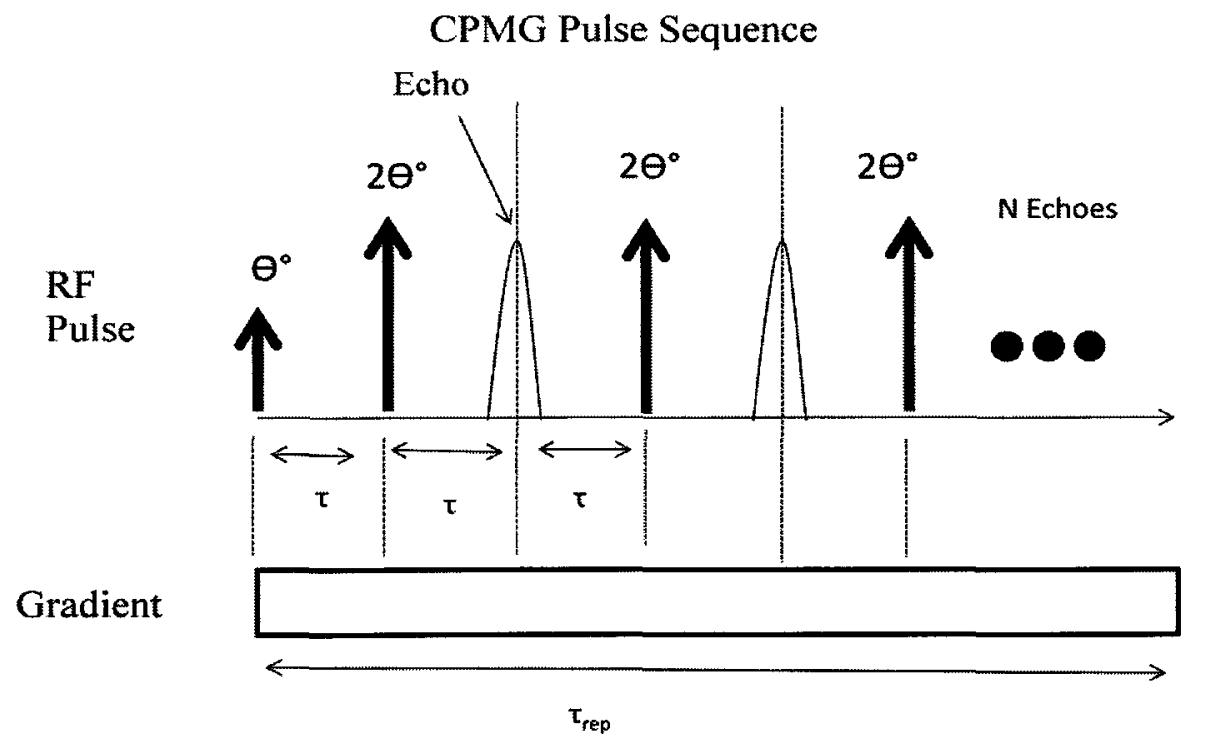

Figure 4 - A typical CPMG Pulse sequence where $\tau$ is the time between pulses and $\tau_{\text {rep }}$ is the time between scans. The echo shown in the RF pulse line is the signal that is measured. 


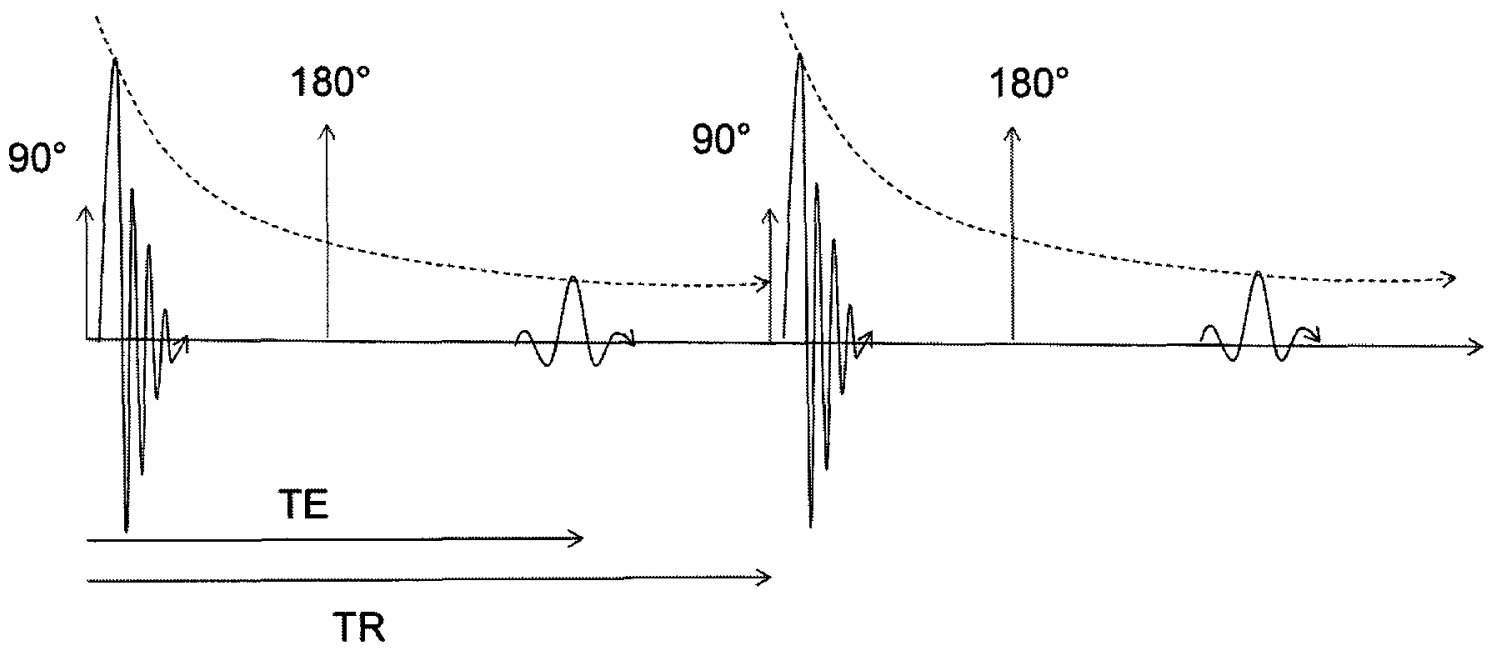

Figure 5 - The peaks of the echoes that are formed after the $180^{\circ}$ refocusing pulse obeys T2 decay.

\subsection{Basic MRI Image Formation}

As mentioned in section 2.2 , the frequency the dipoles spin at under the magnetic field depends on the location of the dipoles in field. Therefore to create an image using $\mathrm{MRI}$, the system needs to be able to analyze the acquired signal and extract its frequency spectrum. To show this, consider that at position $\boldsymbol{r}$ in the sample will occupy a small volume $d V$. With some derivation, we can show that [6]

$$
S(t)=\iiint p(r) e^{i \gamma G \cdot r t} d r
$$

where $S$ is the signal strenth and $p$ is the proton spin density. We can introduce a wave vector $\boldsymbol{k}$, where

$$
k=\frac{\gamma}{2 \pi} \int G(t) d t=\frac{\gamma G t}{2 \pi}
$$

Substituting Eq. (2.9) in to Eq. (2.8), we can get the Fourier transform pair 


$$
\begin{aligned}
& S(k)=\iiint p(r) e^{i 2 \pi k \cdot r} d r \\
& p(r)=\iiint S(k) e^{-i 2 \pi k \cdot r} d r
\end{aligned}
$$

The acquired signal is obtained in the time domain $S(\boldsymbol{k})$. To obtain the image $p(\boldsymbol{r}), \boldsymbol{k}$ should be sampled through $t$ or by varying the gradient. The $k$-space map generated contains all the information in the field of interest of the precessing spins regarding their location in space and its proton density based on the applied gradients. A typical 3D multi-slice image has one dimension encoded in frequency another in phase and the $3^{\text {rd }}$ dimension via slice selection. Common image contrast parameters are $\mathrm{T} 1, \mathrm{~T} 2$, and proton spin density.
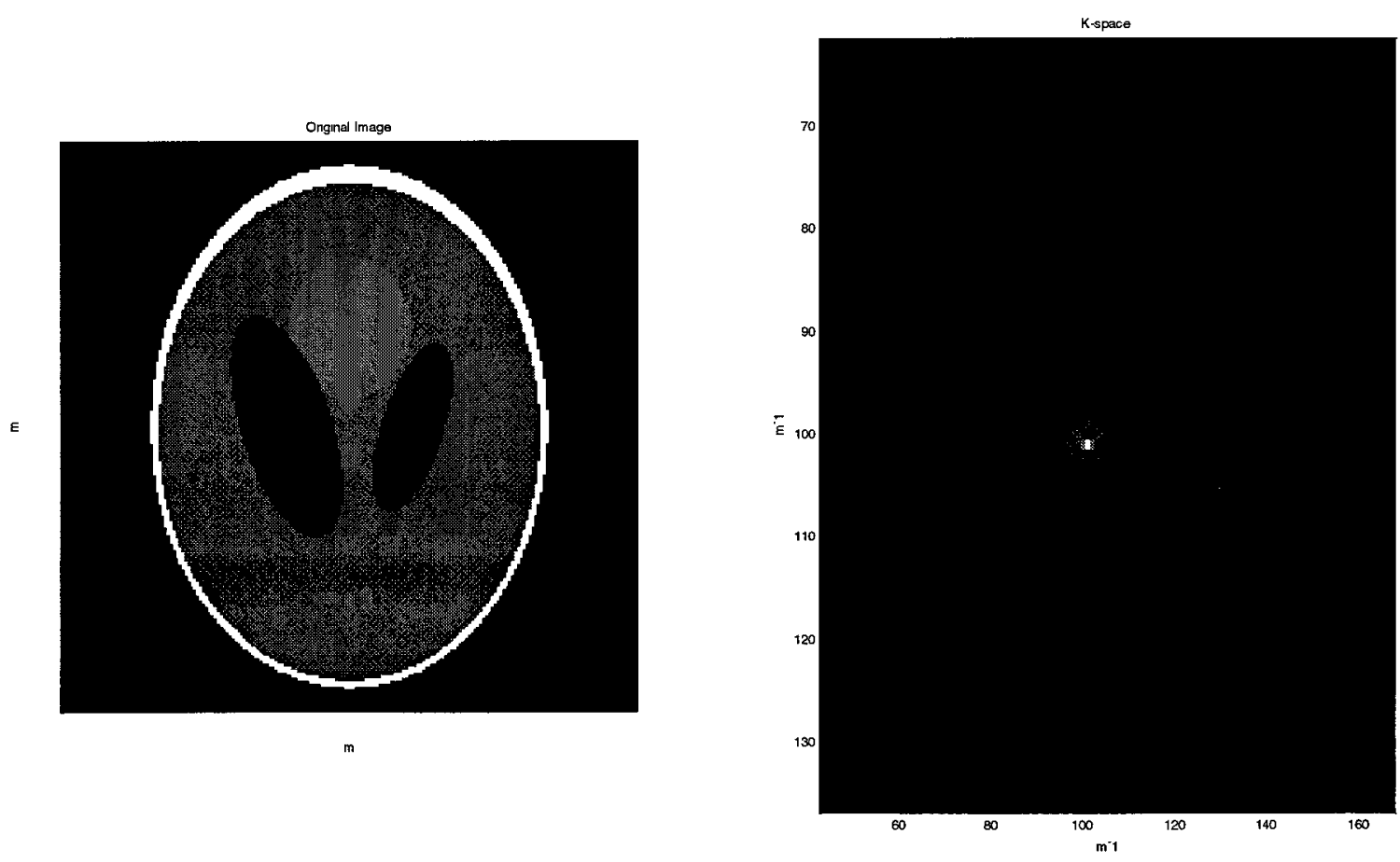

Figure 6 - Matlab generated sliced image phantom and its corresponding k-space frequency map. The center of $k$ space shows the low spatial frequency component. 


\subsection{Velocity Imaging}

Velocity imaging is similar to the idea discussed in the previous section where the main difference is that normal MRI imaging techniques uses the zero-th moment while velocity imaging uses the $1^{\text {st }}$ moment. To explain more clearly, we expand $\boldsymbol{r}$ in Eq. (2.10) as a Taylor series. We can see that the initial position information is then encoded in the zero-th moment, velocity is encoded in the first moment, while acceleration is encoded in the second moment, and onwards to higher order [11]. This is shown in Eq. (2.12).

$$
2 \pi \boldsymbol{k} \cdot \boldsymbol{r}=\boldsymbol{r}(0) \int \boldsymbol{G}(t) d t+\dot{\boldsymbol{r}} \int \boldsymbol{G}(t) t d t+\frac{1}{2} \ddot{\boldsymbol{r}} \int \boldsymbol{G}(t) t^{2} d t+\cdots
$$

If we assume that $\int \boldsymbol{G}(t) d t$ is zero under an oscillating gradient, we can then introduce a new reciprocal velocity space variable $\boldsymbol{p}$ that is sensitive to motion [6], where

$$
p=\gamma \int_{0}^{\tau} t \boldsymbol{G}(t) d t
$$

We can traverse $\boldsymbol{p}$-space by varying the gradient $\boldsymbol{G}$ or sampling in $t$. Performing an inverse Fourier transform in $p$-space will provide us with the velocity distribution. The modified velocity imaging technique used for this experiment will be further discussed in the mathematical model section 4.1.

\subsection{Literature Review in Elastography}

Elastography is a non-invasive imaging technique that can visualize tissue elasticity in-vivo. There are currently two main image modalities used to perform elastography, Ultrasound Elastography (UE) and Magnetic Resonance Elastography (MRE). Other 
elastography technique that are in development are Optical Coherent Elastography (OCE) [12] [13] and X-ray Elastography [14]. The following sub-sections will focus on techniques and application that have been adapted for clinical used with the primary focus on MRE.

\subsubsection{Ultrasound Elastography}

Current Ultrasound Elastography techniques were formed in the 1990's at University of Texas Medical School. This technique can be categorized into two sub-techniques, quasi-static stress model [15] [16] and dynamic stress model [17] [18] [19]. The quasistatic method is based on taking sonograms with and without applied compression on the tissue. The resulting images are then correlated to determine the displacement at each location revealing longitudinal strain distribution.

The dynamic stress method is based on employing a low frequency shear waves on the targeted tissues. Doppler ultrasound is then used to measure the propagation velocity of shear waves. Higher propagation velocity results in a higher Young's Modulus. These methods have been used in many applications such as imaging breast lesions [20], liver [21] [22], and heart [23].

\subsubsection{Magnetic Resonance Elastography}

Current Magnetic Resonance Elastography (MRE) techniques were originally developed by the Mayo Clinic in 1995 for the purpose of detecting various types of liver 
disease, primarily hepatic fibrosis [24]. Current clinical application of MRE consists of studying the blood vessels [25], skeletal muscles [26] [27] [28] [29], heart [30] [31] [32] [33], liver [2] [34] [35] [36] [37], lungs [38] [39], brain [40] [41], breasts [42] [43] [44], and prostate [45]. The following sub-sections will discuss the recent development in MRE in terms of shear wave generation, and wave imaging.

\subsubsection{Shear Wave Generation in Tissue}

MRE requires the use of a single frequency shear wave vibration that is typically generated by an external oscillatory driver in the audio frequency range. The penetration depth of a shear wave is highly dependent on its frequency due to large attenuation caused by tissue viscosity [46]. A low frequency driver $(50-100 \mathrm{~Hz})$ is typically used for breast elastography [42] while a higher frequency driver $(125-150 \mathrm{~Hz})$ is typically used for near skin skeletal muscles [26] or organs that requires higher spatial resolution to detect much smaller tumors. These drivers are typically controlled by a signal generator that is triggered and synchronized to a MR pulse sequence. The four most commonly used mechanical driver systems are the acoustic speaker driver [34] [35], electromechanical driver [24] [47], piezoelectric driver [48] [49] and focused-ultrasoundbased radiation force $[50][51][52][53][54]$.

The acoustic driver uses a loudspeaker for vibration generation that is connected to a passive driver that is placed on the patient body. Mechanical vibration is then transmitted by a pneumatic tube to allow separation of the magnetic components of the loudspeaker 
from the MRI magnet. This type of driver has good MR compatibility, is simple to use and is relatively cheap. However, the key issues are the phase lag in mechanical motion and synchronization. Both issues are intrinsic problems of a pneumatic system [46].

The electromechanical driver uses the $B_{0}$ magnetic field generated by the main magnet to induce a torque in the coils of the driver when a current is applied. This type of driver is relatively inexpensive and simple in terms of design and can generate a wide range of frequency. However, the device is not robust in terms of orientation and the ability to easily change the vibration amplitude. Also, image artifacts can occur due to the currents in the coil that can disturb the main field.

The piezoelectric driver uses a piezoelectric material that is placed near the region of interest. When a current is applied, the material bends generating a force. Although the piezoelectric vibrators are stable, MR compatible (non magnetic) and can be easily controlled for synchronization, its small displacement (in $\mu \mathrm{m}$ ) is an issue that it is much less than the resolution of the MRI. Safety is also of a concern as high voltages are employed to these vibrators that are designed to touch the patient's skin directly.

The ultrasound-based radiation force driver can generate transient shear waves near the focal spot due to cyclic modulation of radiation pressure [50]. The advantages of using ultrasound are that it is MR compatible, penetration depth and focus location of the generated shear waves in-vivo can be easily control, it does not suffer from shear wave tissue attenuation as the radiation force is created internally. The main limitations of this 
technology in terms of safety and effectiveness are tissue heating, cavitation and that an acoustic path is needed to reach the target organ [53].

\subsubsection{Wave Imaging - MRE Pulse Sequences}

Current MRE imaging technique is typically a phase-contrast imaging technique that can quantitatively measure propagating acoustic shear waves that are subjected to harmonic force oscillations [24]. A typical MRE setup uses a trigger pulse that synchronizes a motion-encoding gradient (MEG) along specific direction and an oscillatory unit that drives an actuator [55] [56] [25]. The resulting phase shift in the presence of the motion-encoding gradient is given by [57]

$$
\varphi(r, \alpha)=\frac{\gamma N T\left(G_{o} \cdot \varepsilon_{0}\right)}{2} \cos (k \cdot r+\alpha)
$$

where $\gamma$ is the gyromagnetic ratio for protons, $\mathrm{N}$ is the number of cycles of the oscillating field gradient, $T$ is the period of mechanical excitation and oscillating gradient, $\boldsymbol{G}_{\boldsymbol{o}}$ is the vector of the gradient amplitude, $\varepsilon_{o}$ is the vector of the mechanical displacement amplitude, $\boldsymbol{k}$ is the wave vector, $\boldsymbol{r}$ is the position vector and $\alpha$ is the phase difference between mechanical excitation and the MEG. The motion-encoding capability of the technique proposed by [57] can detect motion in the order of 100's of nanometers.

The only major difference between MRE and conventional MRI imaging techniques is the introduction of an external vibration source and the introduction of a motionencoding gradient. However, MEG can be implemented by using MR imaging sequence such as fast spin echo (FSE-MRE) [58], balanced steady-state free precision [59], mutli- 
echo phase contrast gradient-echo sequence (ME-PC-GE) [60] and compliance-weighted imaging (CWI). Each sequence has its own advantages and disadvantages. The FSEMRE is an adaptation of the fast spin-echo where a series of $180^{\circ}$ rephrasing pulse is applied and where the phase encoding gradient changes at each echo. The key change for application in MRE is that the motion encoding is performed between the first two $180^{\circ}$ RF pulses. The main advantage of this sequence is that it can potentially improve the speed of acquisition of data over typical spin-echo and gradient-echo sequences. The balanced steady-state free precision has the advantage of generating high sensitivity for steady-state phase and amplitude images because of its use of the read gradient as the MEG which can also lead to improve scan-time efficiency. However, quantitative information about mechanical displacement cannot be solely extracted from the steadystate phase and amplitude thus requiring other parameters such as off-resonance distribution and relaxation times. The ME-PC-GE sequence generates a user-defined number of echoes per RF excitation. Blip gradient in the phase encoding are then used to fill k-space with repeated TR. The sequence can drastically improve scan time but would also lower the image quality. Compliance-weighted imaging (CWI) is method that utilizes very low frequency vibration that can be used to measure relative stiffness of subcortical gray matter.

The MEG frequency does not always have to match the frequency of motion. It has been shown that by using a multiple of the motion frequency, acquisition times can be drastically reduced due to the decrease in echo time (fractional encoding) [61]. This is most effective when measuring tissues with very short $\mathrm{T} 2$. A disadvantage is that it will 
have lower motion sensitivity. An improvement to fractional encoding is to use displacement encoding with stimulated echoes (DENSE) [30]. It is more sensitive to displacement than fractional encoding of harmonic motions, and its spectral specificity is equivalent to standard MRE sequences. Another group have also attempted to induce multiple frequency shear waves into the body and used a broadband motion sensitizing gradient [35]. The advantage of this is that it can provide higher-order models for visualizing tissues which can provide more information during diagnosis.

\subsubsection{Elastography Comparison and Improvements}

Both elastography modalities have their own advantages and disadvantages. The advantages of MRE is that it can analyze a whole volume and provide a three dimensional reconstruction tissue elasticity whereas UE technology currently can provide a higher accuracy for measured elasticity locally for a much smaller sample size [37] [62]. The main drawback to MRE compared to UE is the cost of the equipment in performing these measurements [63]. A potential way to reduce the cost of current MRE systems is the use of portable magnets (section 2.8).

\subsection{Single-Sided Magnets Literature}

Single-sided magnets make use of an open configuration of magnets to produce a MR compatible static field in an external volume. Compared to conventional MR systems, single-sided magnets are typically used for bulk MR measurements to estimate 
parameters such as $\mathrm{T} 2, \mathrm{~T} 1$, and self diffusion in arbitrary sized samples. The challenge in creating these magnets is the generation of a large volume with a uniform gradient. In the past, low cost portable magnets have been developed such as a $1 \mathrm{D}$ palm-size probe [64] and an open top single sided NMR [65]. These systems have been shown to be able to remotely measure velocity [66], produce a two [67] and three dimensional image of phantoms [68], and measure self-diffusion [10]. A more recent unilateral NMR magnet design has a larger measurement volume and a more homogeneous magnetic field [69] [70]. Systems based on this new design have been used for near-surface MRI profiling [71] and determination of fat content in packaged dairy products [72]. 


\section{MRE Hardware}

This chapter will present the equipment used for this thesis. In addition, the chapter will discuss in detail the specifications of the equipment. A block diagram of the overall system used in this work is shown in Figure 7. The MRE System is composed of the single-sided magnet and a propagating medium. Details on the evolution of this setup is discussed throughout chapter 5 of this thesis. The position system is used to precisely control the vibration source, the Bruker system is used to generate pulse sequences and to acquire MRI signal and the computer is used to process and display the data.

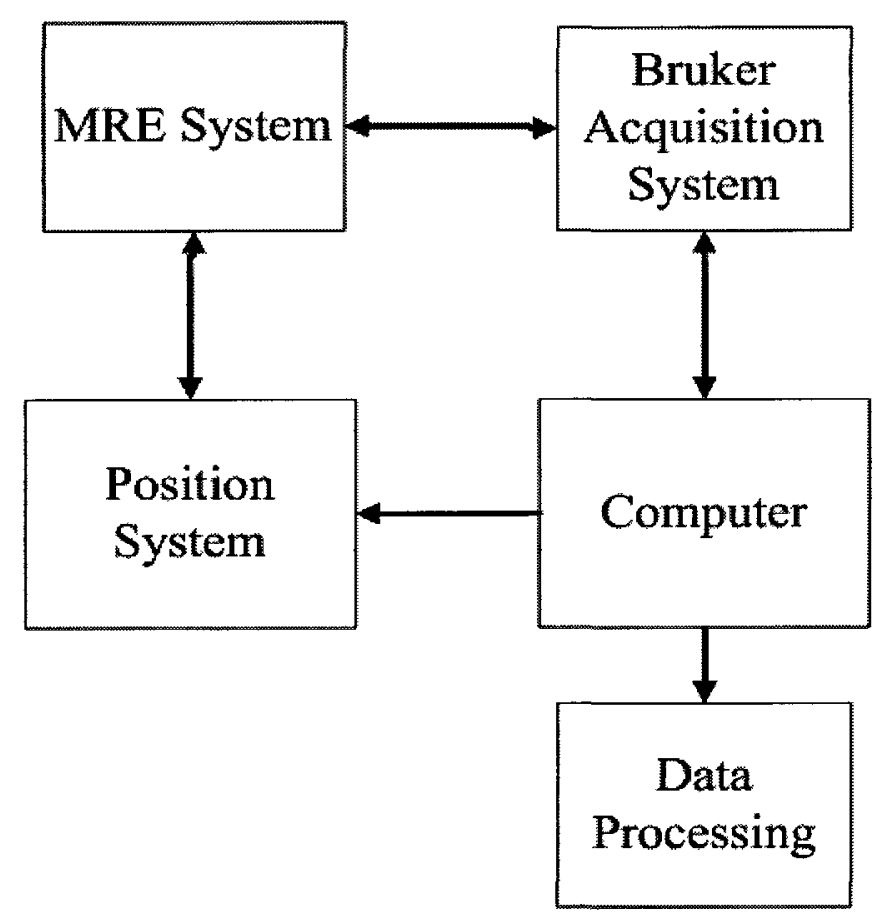

Figure 7 - A block diagram of the experimental setup used in this thesis. 


\subsection{MRI Pulse Synthesizer Hardware Specifications}

The MRI system is operated by the Bruker Optics NMR spectrometer which consists of a pulse synthesizer and an amplifier [73]. The data is captured and recorded from the RF coil using Bruker's ExpSpel software running on a Windows XP operating system. The Bruker Optics NMR system used can be seen in Figure 8. The following sub-section will present the scanning parameters that can be set using the Bruker system.

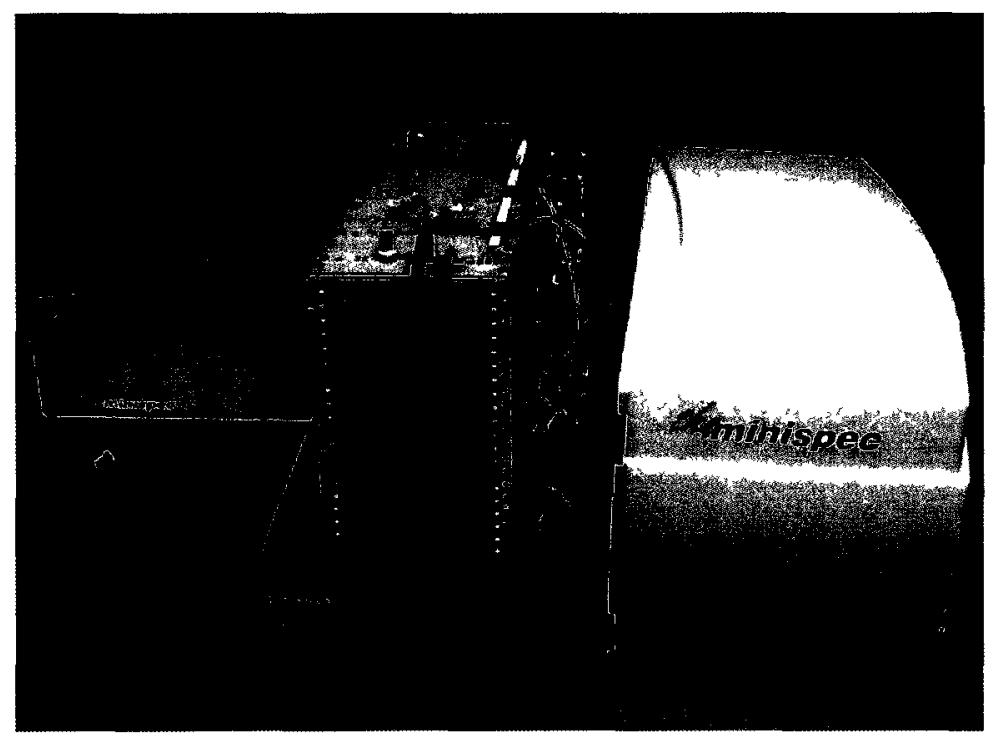

Figure 8 - Bruker Minispec System. The preamplifier is the small box on the left and the RF pulse generator is shown on the right.

\subsubsection{Bruker Optics NMR Scanning Parameters}

For this research, all measurements were conducted using variations of CPMG pulse sequences. There are five parameters that were modified in creating the pulse

sequence: Number of Scans $\left(\mathrm{N}_{\text {scans }}\right)$, Acquisition time (acq), Points per Echo (PPE), and 
Number of Echoes ( $\mathrm{N}_{\text {echoes}}$ ). $\mathrm{N}_{\text {scans }}$ is used to increase the signal-to-ratio by $\sqrt{\mathrm{N}_{\text {scans }}}$ [74]. ACQ is the length of time over which the echo is sampled. PPE determines the number of points that are sampled in one echo. $\mathrm{N}_{\text {echoes }}$ defines how many $180^{\circ}$ pulse trains are generated in one pulse sequence. Having a higher number of echoes improves the sensitivity of the experiments [10]. The number of data points and number of echoes are limited by the internal memory of the Bruker system to approximately 64000 points per scan.

\subsection{Single-Sided Magnet}

The magnet used for this experiment is described in [70]. The magnet was created using three block array magnets magnetized along the same direction. The magnet measures $10 \mathrm{~cm}$ by $11.5 \mathrm{~cm}$ by $6 \mathrm{~cm}$ and weighs approximately $5 \mathrm{~kg}$. A surface coil tuned to a frequency of $4.545 \mathrm{MHz}$ is then mounted $\sim 5 \mathrm{~mm}$ above the center of the magnet array. The design is optimized so that there is relatively homogeneous area of the magnetic field $B_{0}$ of $0.1 T, 1.05 \mathrm{~cm}$ above the face of the magnet. Above this 'sweet spot', it was measured that the there is a slight upward trend in field strength before decaying at a relatively uniform gradient of approximately $1.09 \mathrm{~T} / \mathrm{m}$ along the $B_{1}$ direction. The magnet has a resolution of one pixel of approximately $0.5 \mathrm{~cm}^{3}$. A schematic diagram of the magnet is shown in Figure 9 and the magnet is shown in Figure 10. One of the main difference between the single-sided magnet used for this experiment and conventional MRI magnets is that the gradient is a constant and inherent feature of the static field, and is thus indicated as always on. In contrast, the magnetic field of a 
conventional MR system contains a gradient that can be switched on and off, and can be adjusted both in magnitude and direction.

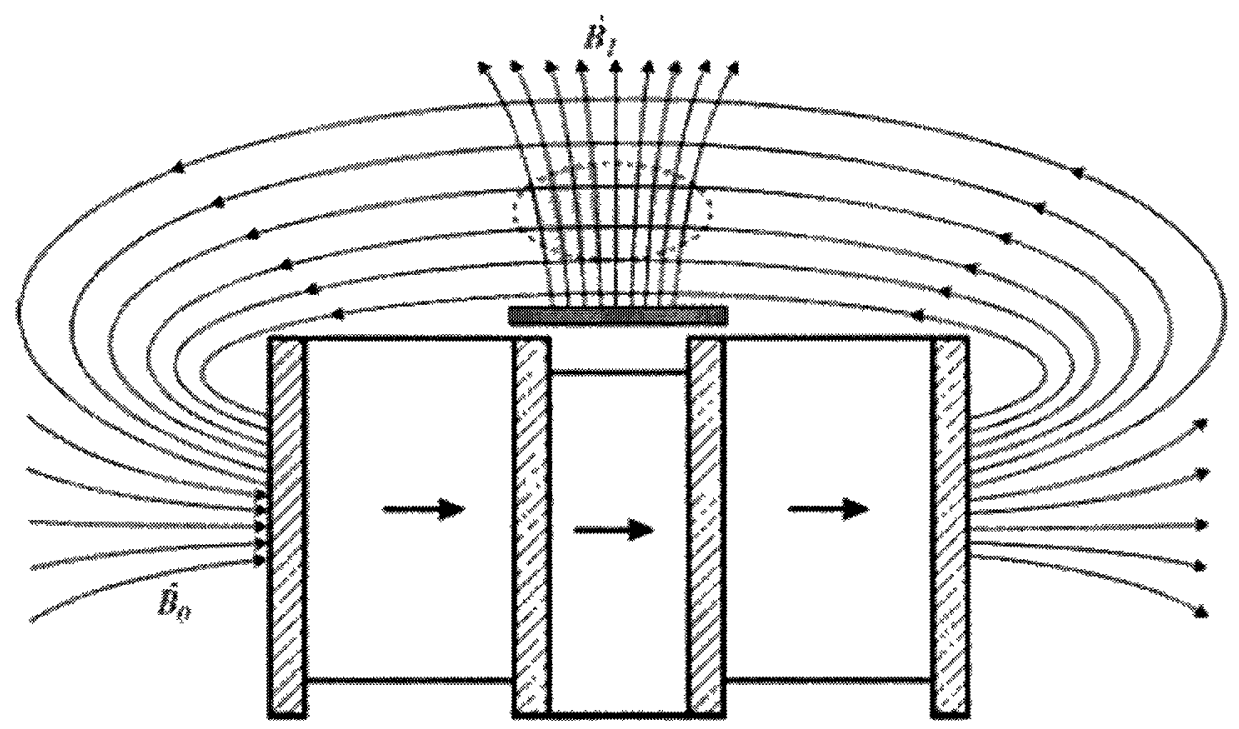

Figure 9 - A Schematic Diagram of the single-sided magnet used for this work. The shaded region is the "sweet spot" of the magnet where there is a relatively constant magnetic field gradient $B_{1}$. Reproduced from [72]. 


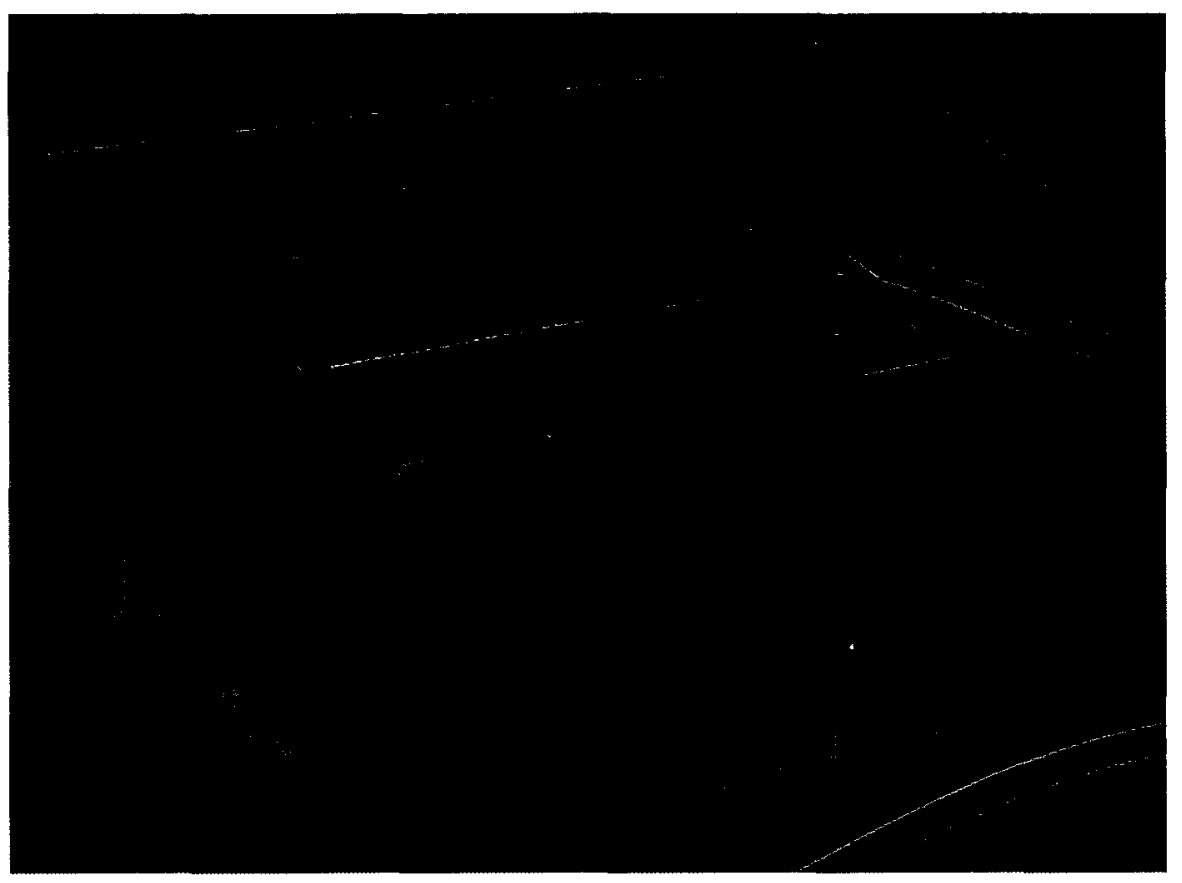

Figure 10 - Single-sided magnet used for this work.

\subsection{Positioning System}

The positioning system used to control the massager in our experiment is the Velmex BiSlide system (Fig. 11) [75]. It is composed of a BiSlide MN10-0300-E01-31(ydirection), 2x Bislide MN10-0300-E01-21 (x-direction), a BiSlide MN10-0300-E01-20 (z-direction), a Framing Table and a VXM-3 Control. For this research, the system is controlled by the Bruker's expel software. It is also possible to manually adjust the system using the VXM motor controller. 


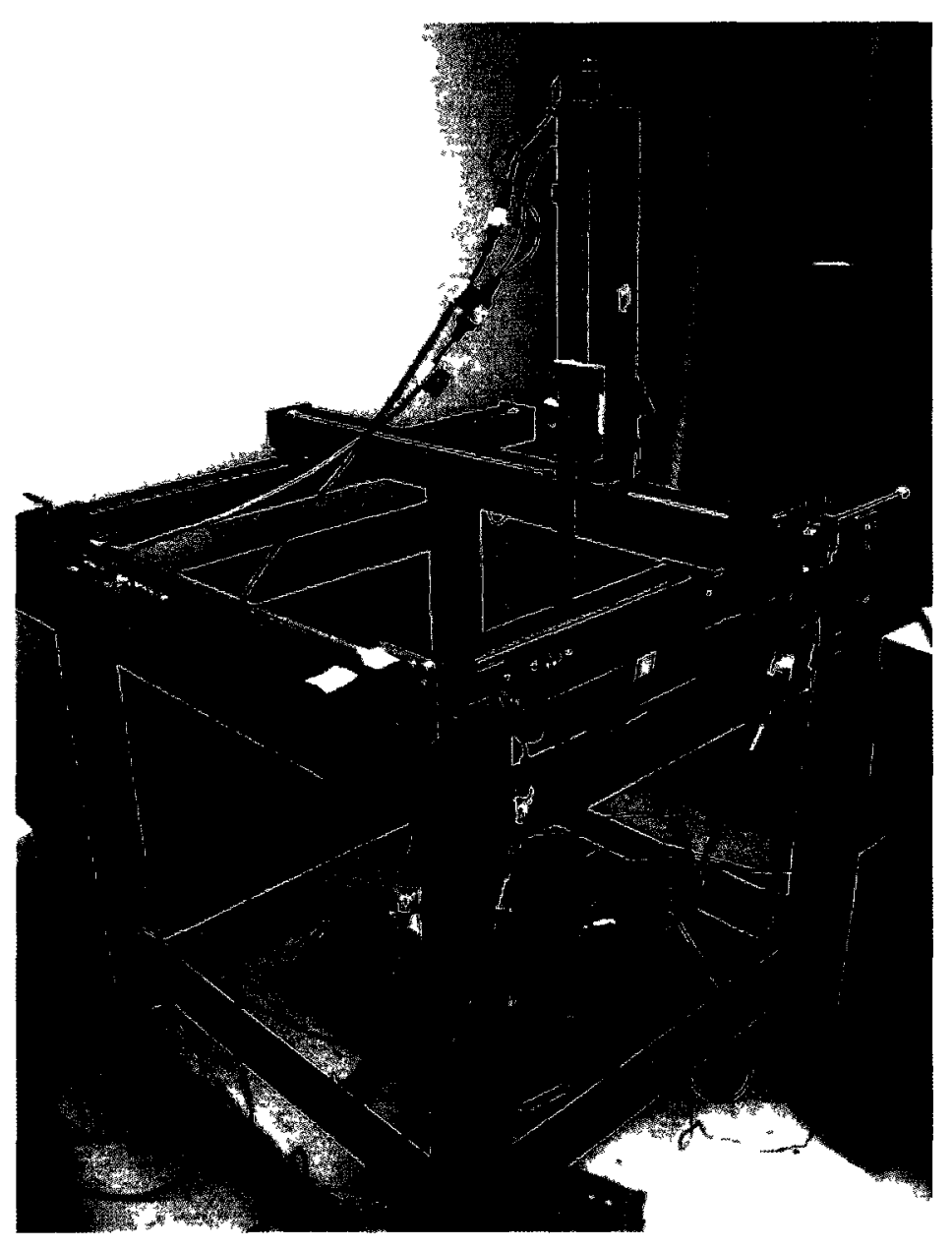

Figure 11 - The Velmex position system as setup in the lab.

\subsection{External Vibration Source}

For this work, we used three different devices to act as an external vibration source for the system. The first massager (Conair Model HM24C) was bought from a local drugstore (Fig. 12). It only has one vibration speed which is controlled by the on/off switch. It has a rotating motor that produces the vibration and these vibrations are transmitted through two different ends of the massager. Using a piezoelectric sensor from Piezo Systems INC. (KGS-006 Piezo Generator/Sensor Kit), a material that generates voltage proportional to its applied stress, we measured the vibration frequency 
of both ends and found that one end vibrates at approximately $59 \mathrm{~Hz}$ and the other at approximately $55 \mathrm{~Hz}$. The resulting waveform observed by the oscilloscope for the 59 $\mathrm{Hz}$ vibration side is shown in Figure 13.

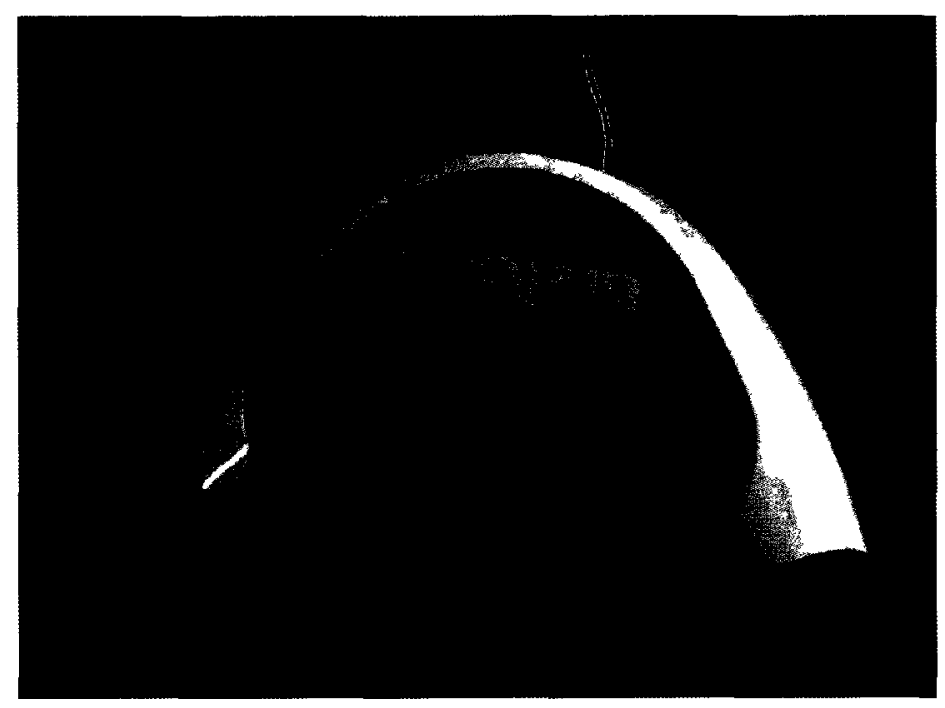

Figure 12 - Conair Massager with the labels $L$ (large frequency) and S (small frequency) to identify the two different frequencies it can generate.

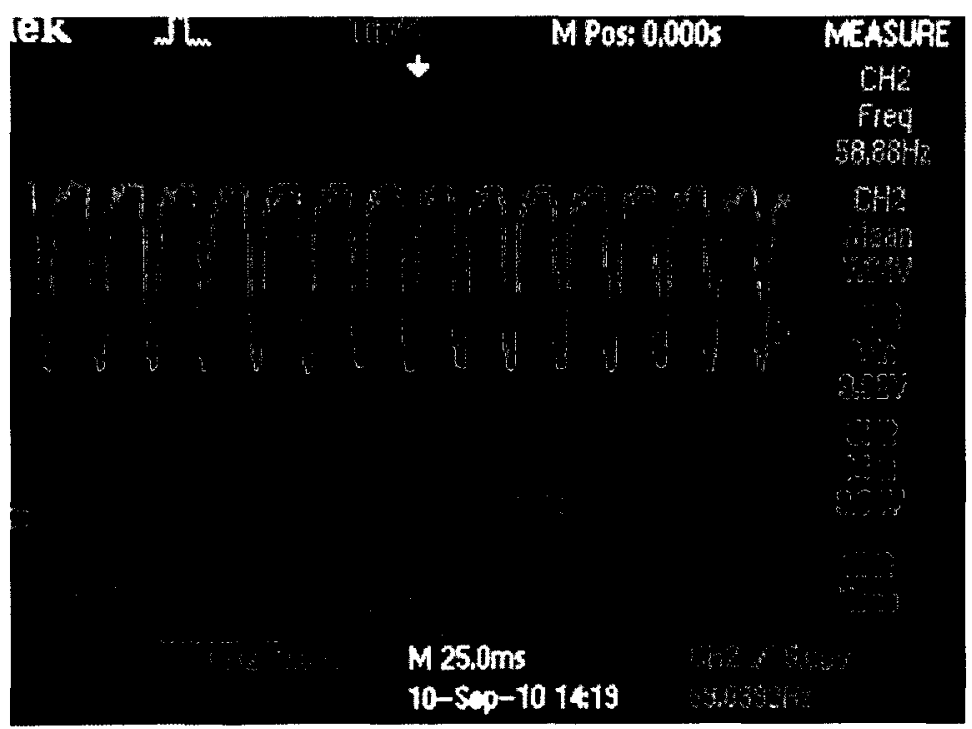

Figure 13 - Output show on a Tektronix Digital Oscilloscope from piezoelectric sensor when the " $L$ " side of the Conair Massager is applied. We can see that the vibration frequency is approximately $\mathbf{5 9 H z}$. 
The second massager used in this thesis is a Dr. Scholl's Hand-held Massager (Model DR7565) shown in Figure 14. Unlike the first massager, this massager has many different type of vibration it can generate on the head of the massager. For our work, we decided to use the top surface head of the massager as it provides the most convenience in controlling the force that will be applied on the sample using the Velmex position system. It has two different speeds, a low-speed and high-speed vibration mode. The vibration frequency for each speed was tested with a piezoelectric sensor similar to obtaining the vibration frequency of the first massager. At low speed the vibration frequency is approximately $50 \mathrm{~Hz}$ and at high speed the vibration frequency is at approximately $60 \mathrm{~Hz}$.

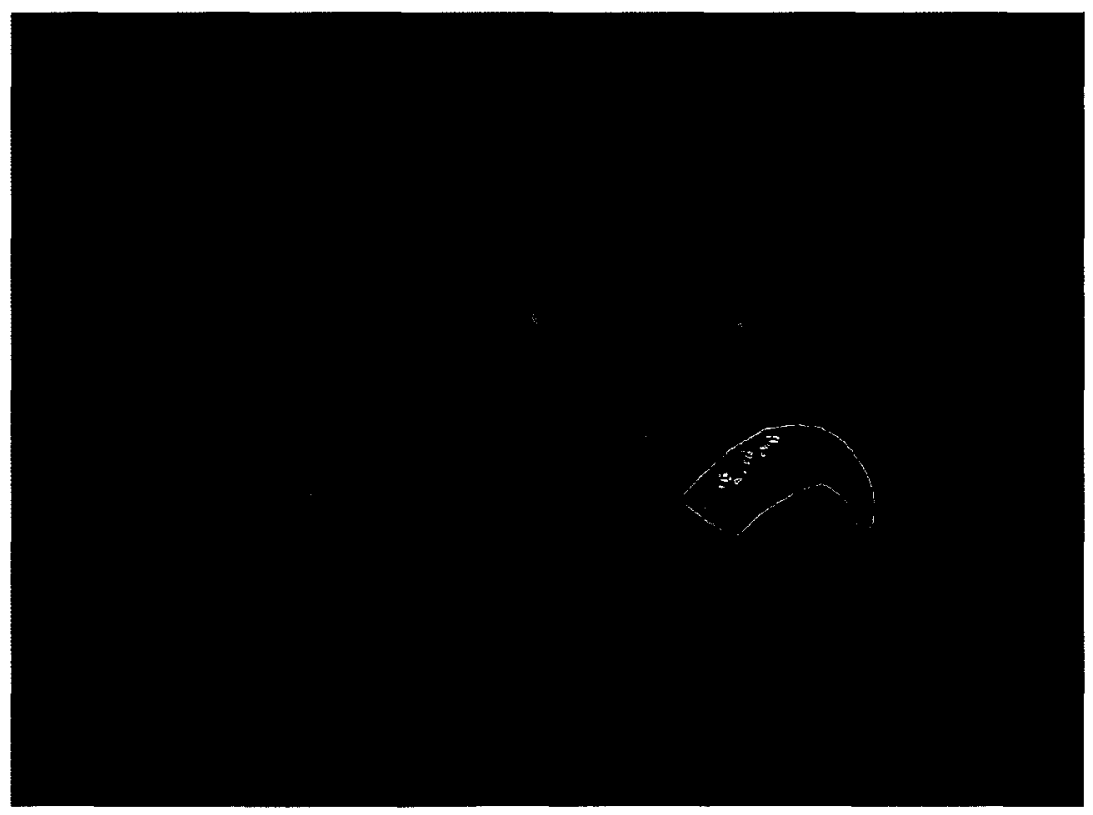

Figure 14 - The image above is the Dr. Scholl's Hand-held Massager (Model DR7565). For the work done in this thesis, we used the top surface of the massager as the vibrating source. 
Note that for both massagers, constant usage will lead to a change in vibrating frequency and amplitude due to heating of the motor over a long period of time. After approximately 100 hours of usage, the massager's vibration frequency has decreased by approximately $10 \%$.

Lastly, a piezoelectric material was attempted to be used as a vibrating source for the MRE measurements as it is non-magnetic and has minimal affect on the MRI signal. The vibration frequency is easily controlled by a signal generator. However, the displacement it generates is a major problem as discussed in section 2.7.2.1. 


\section{Velocity Measurements and Pulse Sequences}

The main idea developed in this work is that a sample oscillating through a magnetic field will experience varying frequency given by the Larmor frequency equation (Eq. 2.4). In turn, this will cause phase dispersion between spins which then leads to echo signal attenuation. To explain more clearly, the following section will provide step by step information in processing the raw signals into data that can give us material elasticity and the sequences to obtain the raw signal. Also, this section will discuss the pulse sequences used in this thesis and the modifications that were proposed to improve signal quality.

\subsection{Velocity Measurements}

During an MRI scan, the RF coil will detect a bulk measurement of spins where the volume is determined by the resolution of the magnet. Under no motion, the spins in that volume will precess at a given Larmor frequency (Eq. 2.4). Applying a CPMG pulse sequence on this sample will generally give us constant $T_{2}$ decay information regardless of the $\tau$ as discussed in section 2.3.2. However when motion is applied, the spins in that sensitive volume will now precess at a frequency that is dependent on the gradient $B_{o}$ and its current position while oscillating. If we look at it from the point of view of a single proton, the proton will experience an oscillating gradient which in turn will constantly vary its frequency. The bulk measurements for these spins under motion will now experience a loss of phase coherence as the spins in the sensitive volume will now 
precess at various frequencies. This in turn causes signal attenuation in the echoes that are formed by the CPMG pulse sequence.

The signal attenuation caused by motion is minimal at very low $\tau(\sim 0.2 \mathrm{~ms})$ for 60 $\mathrm{Hz}$ oscillation frequency. This is true because $\tau$ at $0.2 \mathrm{~ms}$ is very short compared to the period of mechanical oscillation of $\sim 17 \mathrm{~ms}$ at $60 \mathrm{~Hz}$, therefore there is minimal time for the phase dispersion to occur before refocusing. As $\tau$ increases, there is more time for the phase difference to accumulate before refocusing, which will cause an increase in signal attenuation. If the applied mechanical oscillation increases to $120 \mathrm{~Hz}$, the refocusing time will need to be much smaller $\sim 0.1 \mathrm{~ms}$ to observe minimal signal attenuation.

To measure this signal attenuation quantitatively, velocity measurements were used. Recall from section 2.6 , we discussed that motion can be obtained using " $p$-space" sampling where $p=\gamma \int_{0}^{\tau} t \boldsymbol{G}(t) d t$. However, this is only valid if the integrals of the other moments from Eq. 2.12 are zero. A way to achieve this is to have an oscillating gradient field or by applying a CPMG echo train to effectively create an alternating gradient in which the integral of $\boldsymbol{G}(t) d t$ will be equal to zero. The MR system we employed has a fixed gradient, so an alternating gradient method is not possible. Therefore, we chose to apply a CPMG echo train to obtain MRI signal sensitive to velocity. It was discussed that $\boldsymbol{p}$-space can be transverse by varying the gradient or by time. The gradient on the single-sided magnet is constant; therefore we can then evaluate the integral in Eq. 2.13. 


$$
p=\gamma \int_{0}^{\tau} t \boldsymbol{G}(t) d t=\frac{1}{2} \gamma G \tau^{2}
$$

This equation is used throughout this thesis in sampling the MRI signal.

\subsection{Pulse Sequence}

There are two pulse sequences that are used in this thesis, CPMG and Static Gradient Spin Echo (SGSE) CPMG pulse sequences. CPMG was initially used in the preliminary experiments where $\tau$ can vary from $0.2 \mathrm{~ms}$ to $4 \mathrm{~ms}$. The main drawback of using CPMG is the repetition time TR increases drastically as $\tau$ increases. As an example, if an experiment was conducted using 400 scans $\left(\mathrm{N}_{\text {scans }}\right), 600$ echoes ( $\left.\mathrm{N}_{\text {echoes }}\right), \tau$ $=2 \mathrm{~ms}$ the time it takes for the scan to complete would be $\mathrm{N}_{\text {scans }} * \mathrm{~N}_{\text {echoes }} * 2 * \tau$ which is 960 s which is 16 min for one data point. Furthermore, most of the signal occurs within the first $200 \mathrm{~ms}$ of a scan. If we have 600 echoes with at $\tau=2 \mathrm{~ms}$, it would only provide us with 50 echoes during that time span while the remaining 550 echoes would primarily detect noise. The SGSE-CPMG pulse sequence on the other hand saves us a lot of time by setting the echoes at a constant $\tau_{\text {train }}=0.2 \mathrm{~ms}$. The sequence is essentially the same as Fig. 4 except that only the first $180^{\circ}$ pulse varies in $\tau$ (SGSE) while the CPMG Echo Train is always at a constant $\tau_{\text {train }}$ (Fig. 15). Using the above example, the time it takes

for a scan to complete would now be $\mathrm{N}_{\text {scans }} * \mathrm{~N}_{\text {echoes }} * 2 * \tau_{\text {train }}+\tau$ which is a lot faster than CPMG. Furthermore, there will be at least 480 echoes in the first $200 \mathrm{~ms}$ of a scan in comparison to 50 echoes. The minimum time before the next scan can occur was determined experimentally (section 5.2.1). It was found that at TR at $1 \mathrm{~s}$ is optimal to ensure that $M_{z}$ has fully recovered before the next scan can occur (derived in section 5.2.1). In essence, the SGSE portion of the pulse sequence is used to encode motion and 
the CPMG Echo Train is used to improve the signal-to-noise ratio while also providing an effective oscillating magnetic field that is needed for eq 4.1 to be valid (Fig. 16). To effectively perform an inverse Fourier transform, $p$ needs to be sampled uniformly. This is done by initially specifying a range of $p$, and then setting $\tau=\sqrt{p}$.

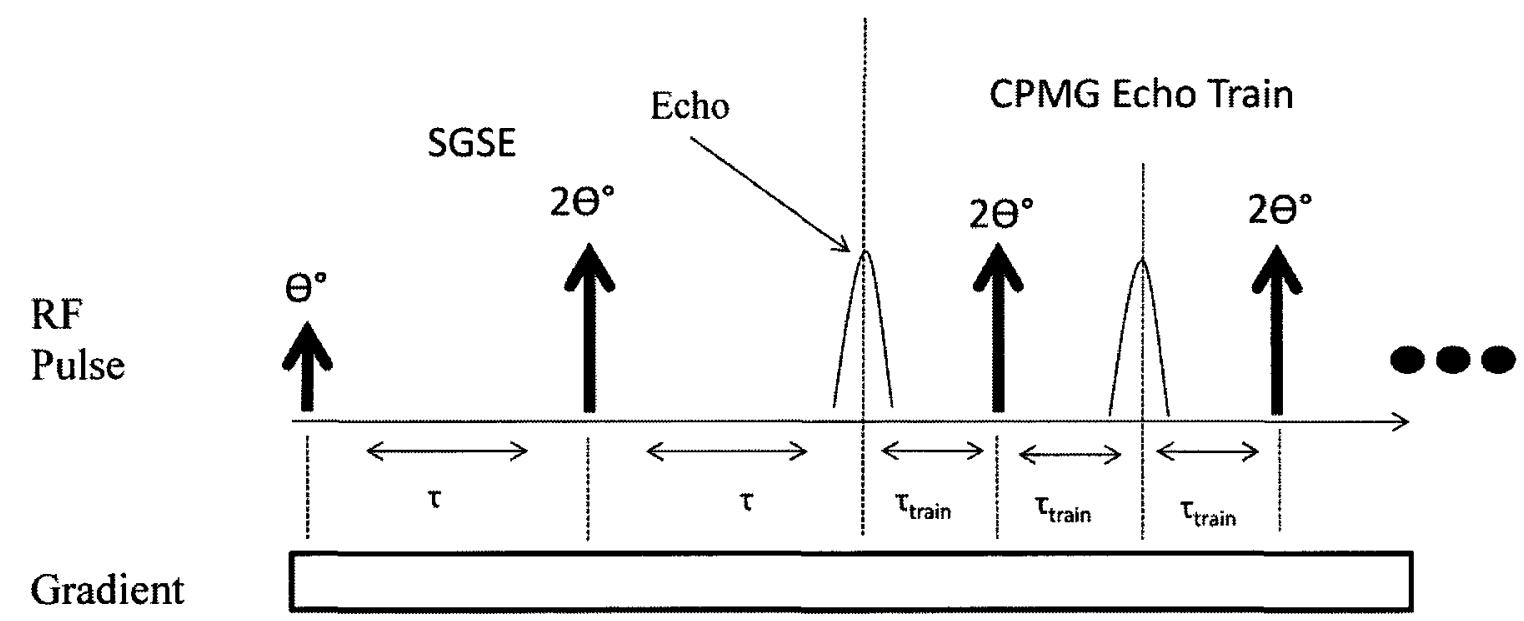

Figure 15-SGSE-CPMG pulse sequence. The SGSE is the part of the pulse sequence that is sensitive to motion. The CPMG Echo Train is there to improve signal-to-noise ratio and to effectively induce an alternating gradient field on the sample. For our work, $\theta$ is $90^{\circ}$. The echo shown in the RF pulse line is the signal that is measured. 


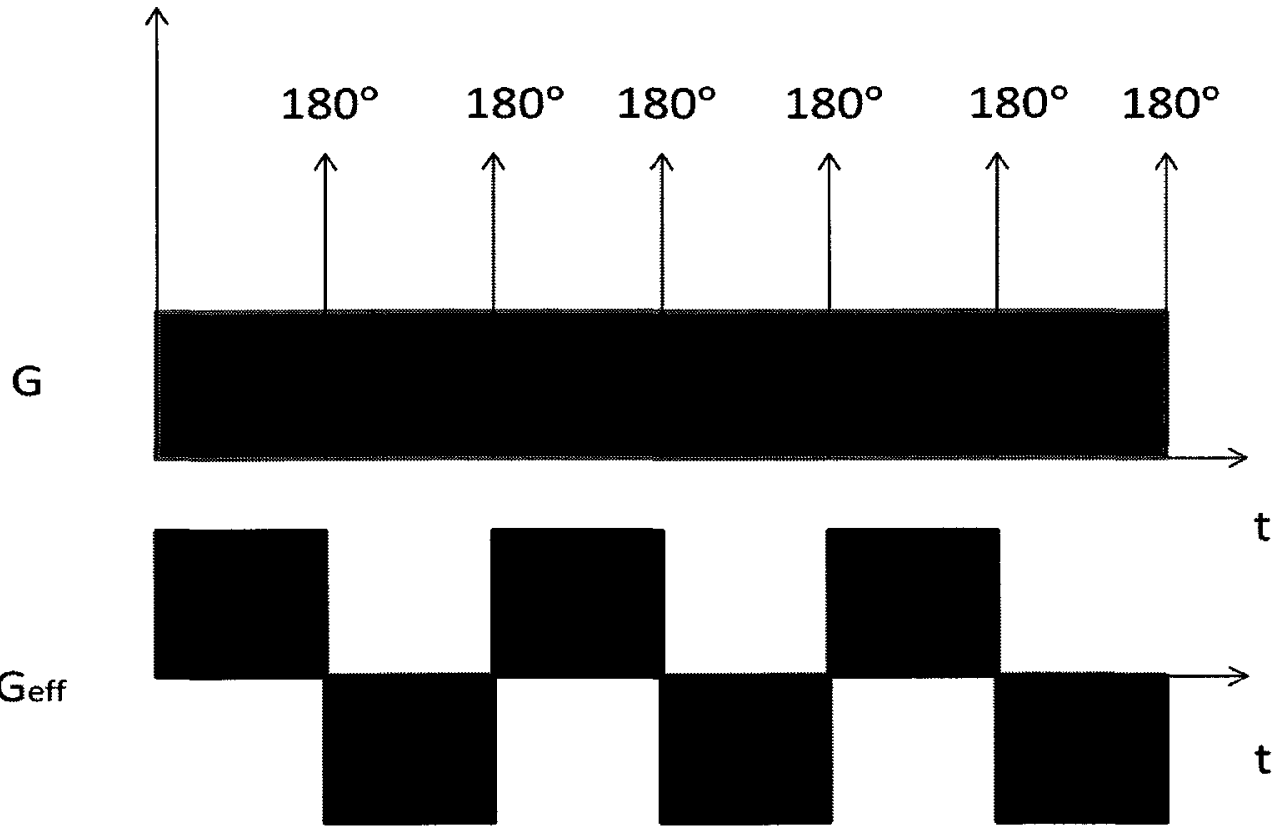

Figure 16 - A CPMG echo train with a static gradient effectively produce an oscillating gradient due to the $180^{\circ}$ pulse flipping the direction of the spins. Flipping the rotation of the spins with a $180^{\circ}$ pulse is the same as applying a negative gradient on the spins.

\subsection{Data Processing Matlab Velocity Measurements}

After a scan has been made and recorded, the data is stored in a text file. A typical scan using the Bruker system is shown in Fig. 17 and 18. 


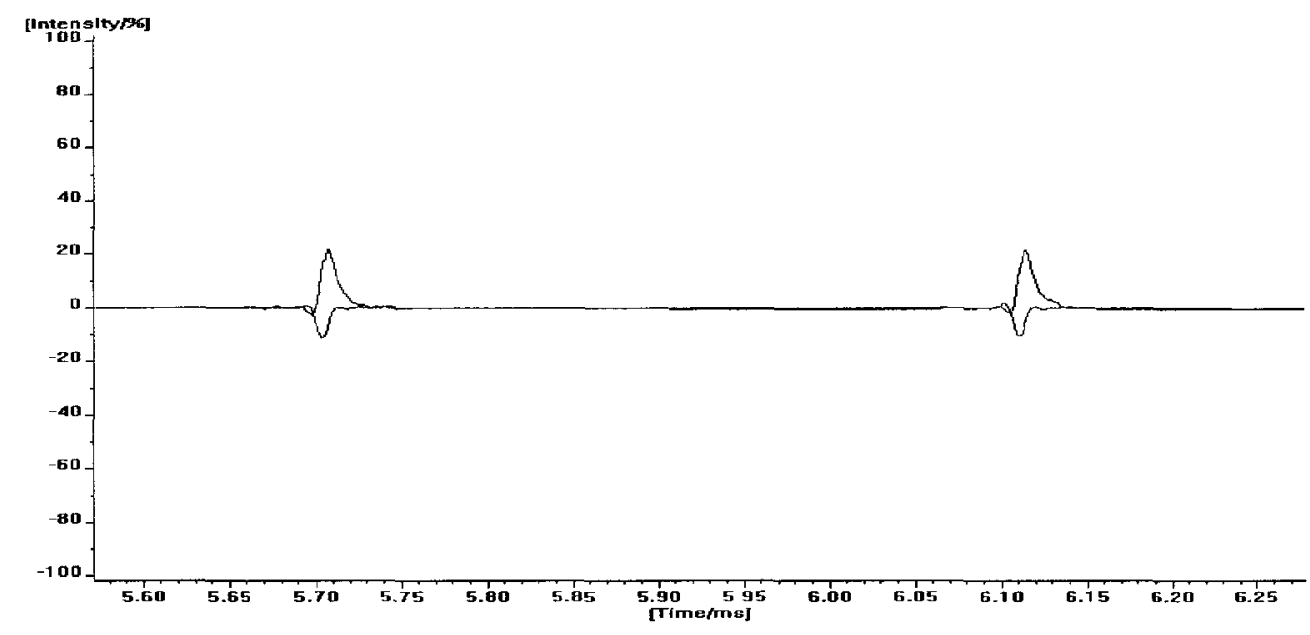

Figure 17 - Raw signal of two echoes obtained using the Bruker system on a $0.225 \mathrm{mg} / \mathrm{L}$ gelatin sample where red is the real data and blue is the imaginary data.

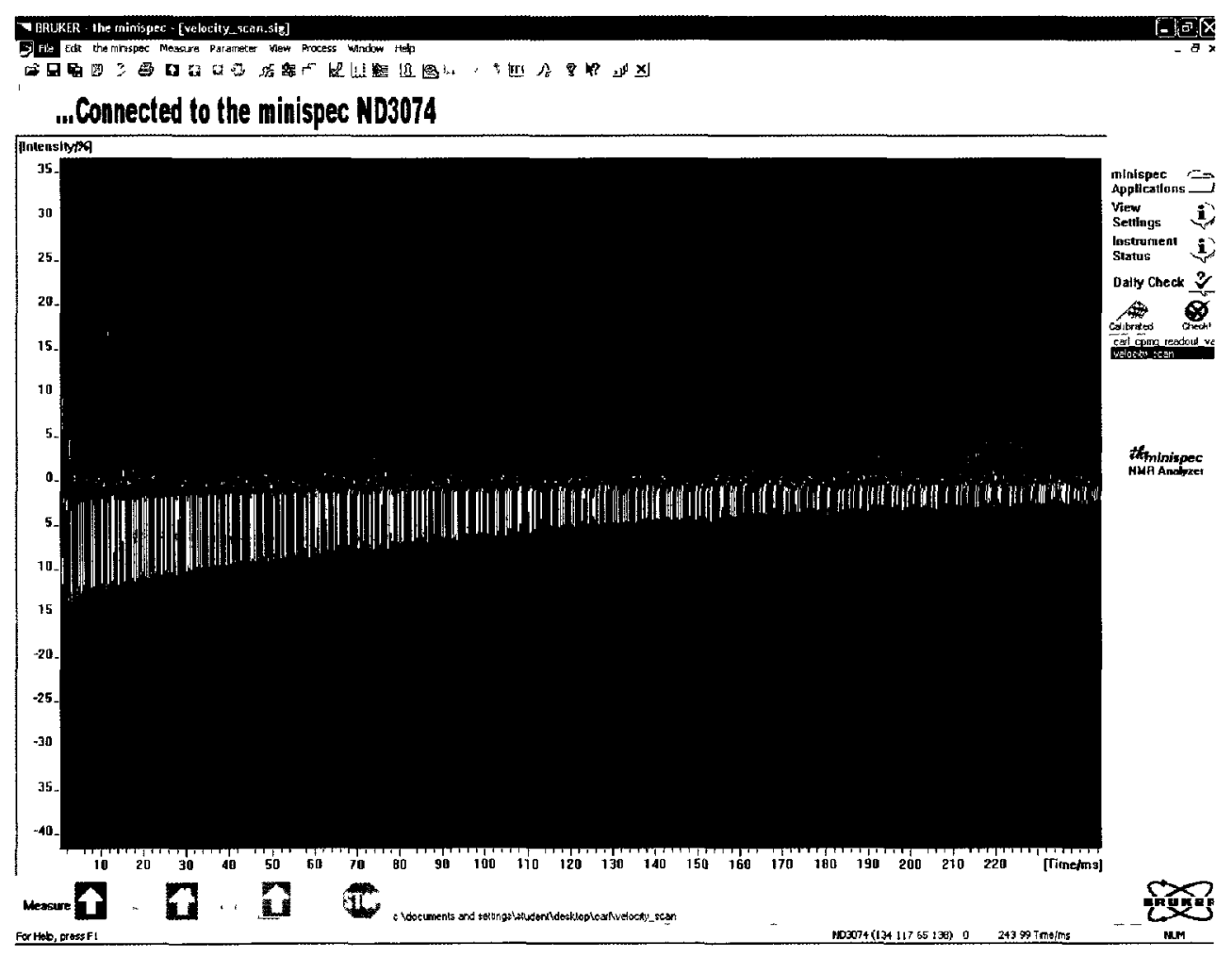

Figure 18 - A Typical MRI (SGSE-CPMG) scan of a $0.25 \mathrm{~g} / \mathrm{mL}$ ethylene glycol gelatin sample obtained using the Bruker system. The scanning parameters were: 400 Scans, 80 Points per Echo, $0.1 \mathrm{~ms}$ Acquisition time, $\tau=0.2 \mathrm{~ms}, \tau_{\text {train }}=0.2 \mathrm{~ms}$ and 601 Echoes. Red is the real data and yellow is the imaginary data. 
All the velocity measurements performed in this thesis were processed in Matlab as follows:

1. Load the data into Matlab. The data is arranged in columns with the following headers: Time, Real, Imaginary.

2. Create another column that contains the magnitude at each given time where magnitude $=\sqrt{\text { real }{ }^{2}+\text { imaginary }^{2}}$

3. For each $\tau$, perform ensemble averaging for all the echoes for the given headers: real, imaginary. An example of ensemble averaging is shown in Fig. 19.

4. Find the index that contains the maximum value in the magnitude. Using that index, obtain the real and imaginary values that correspond to the maximum magnitude data.

5. Repeat Step 4 until all data are processed and all the data are stored in a new array.

6. Plot signal magnitude vs $\frac{1}{2} \gamma G t^{2}$ as $t$ varies with $\tau$. This will provide us the "pspace" plot for the given sample.

7. Using the real and imaginary values of the $p$-space plot, perform an inverse Fourier transform to obtain the velocity probability distribution.

8. Normalize the velocity probability distribution curve so that the integral under the curve is 1 .

For all the SGSE-CPMG used in this thesis, $\tau_{\text {train }}$ is always set to $0.2 \mathrm{~ms}$ because that is the fastest echo repetition time. To quantify the spread of the velocity probability distribution curve, we used root mean square (RMS) velocity where 


$$
V_{r m s}=\sqrt{\frac{\sum_{0}^{n}\left(V_{n} * V p r o b_{n}\right)^{2}}{N}}
$$

where $N$ is the number of points, $\mathrm{Vprob}_{n}$ is the velocity probability and $\mathrm{V}_{n}$ is the velocity at point $n$ of the distribution.
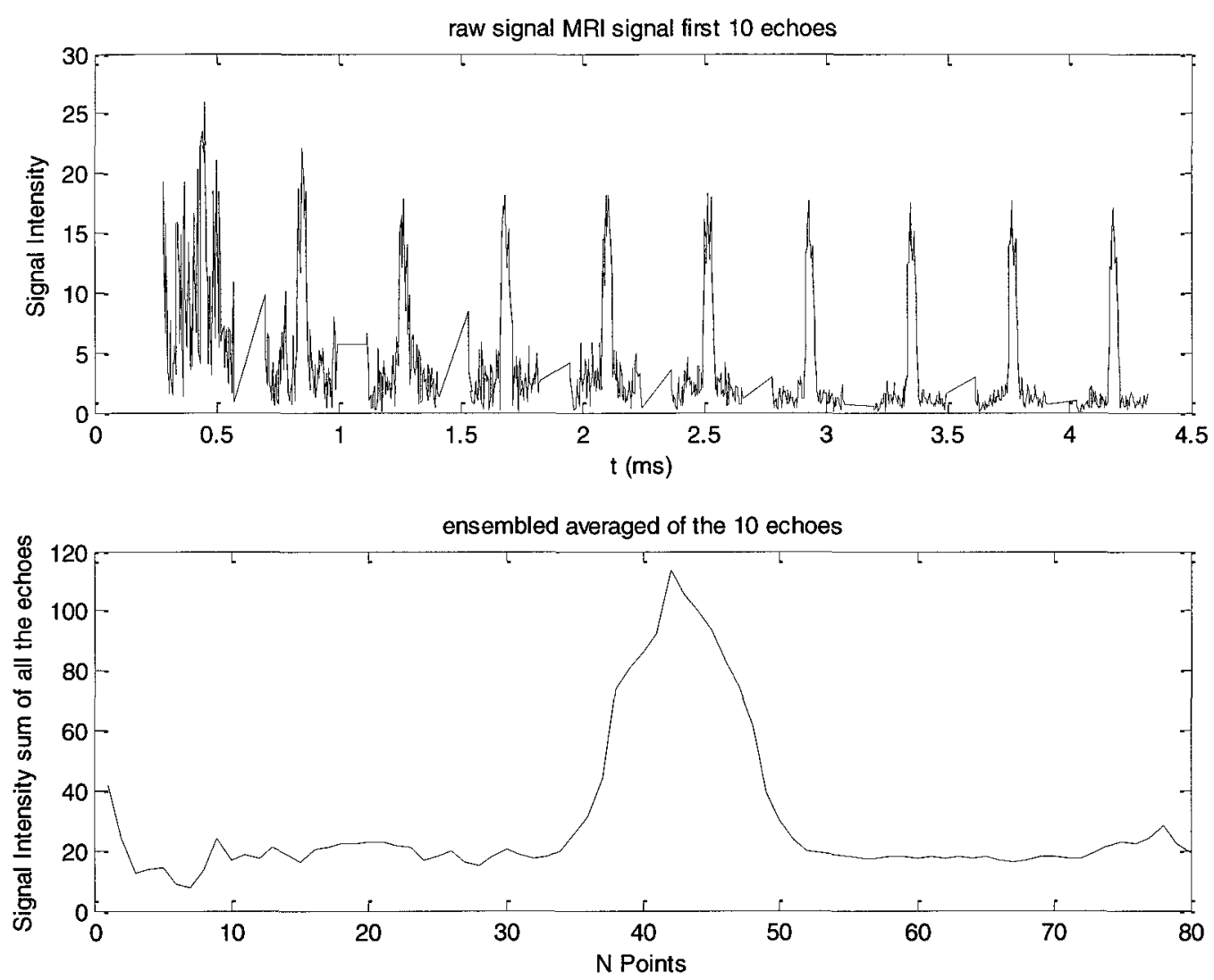

Figure 19- To the top shows raw MRI signal with 10 echoes. Summing all the echoes in the same time sequence will result in an echo shown in the bottom image. To complete ensemble averaging, the figure on the bottom would need to have its signal intensity divided by the number of echoes (10). The $x$-axis of the bottom figure represents the number of data points in each echo that were sampled by the Bruker, the Minispec. In this case, each echo contains 80 points. 


\subsection{Pulse Sequence Optimization}

To improve signal-to-noise ratio, phase cycling and a pulse spacing correction is applied to the pulse sequence generated by the Bruker system. The phase cycling is a modified scanning sequence where the phase of the RF pulse is incremented by a certain angle for each scan. The simplest form of this sequence is a two-phase cycle using a $\left(0^{\circ}\right.$, $\left.0^{\circ}\right)-\left(180^{\circ}, 180^{\circ}\right)$. The purpose of phase cycling is to perform successive phase alternation that leads to successive addition and subtraction of the signal from the MRI. The sum of the data will lead to coherent superposition of the echoes while background noise will be nullified [6]. Typical noises it can eliminate are imbalances in the spectrometer hardware, coherent noise, and artifacts from acoustic ringing. For our experiment, an 8step phase cycling was implemented which was the default phase cycling sequence for the Bruker Software.

A pulse spacing correction of $-2 t_{90} / \pi$ was applied between the initial $90^{\circ}$ and the first $180^{\circ}$ to optimize the timing of the CPMG pulse sequence for maximum signal bandwidth. It has been shown this optimization improves the signal-to-ratio by up to 1.2dB [76]. This is experimentally shown in Fig. 20. 


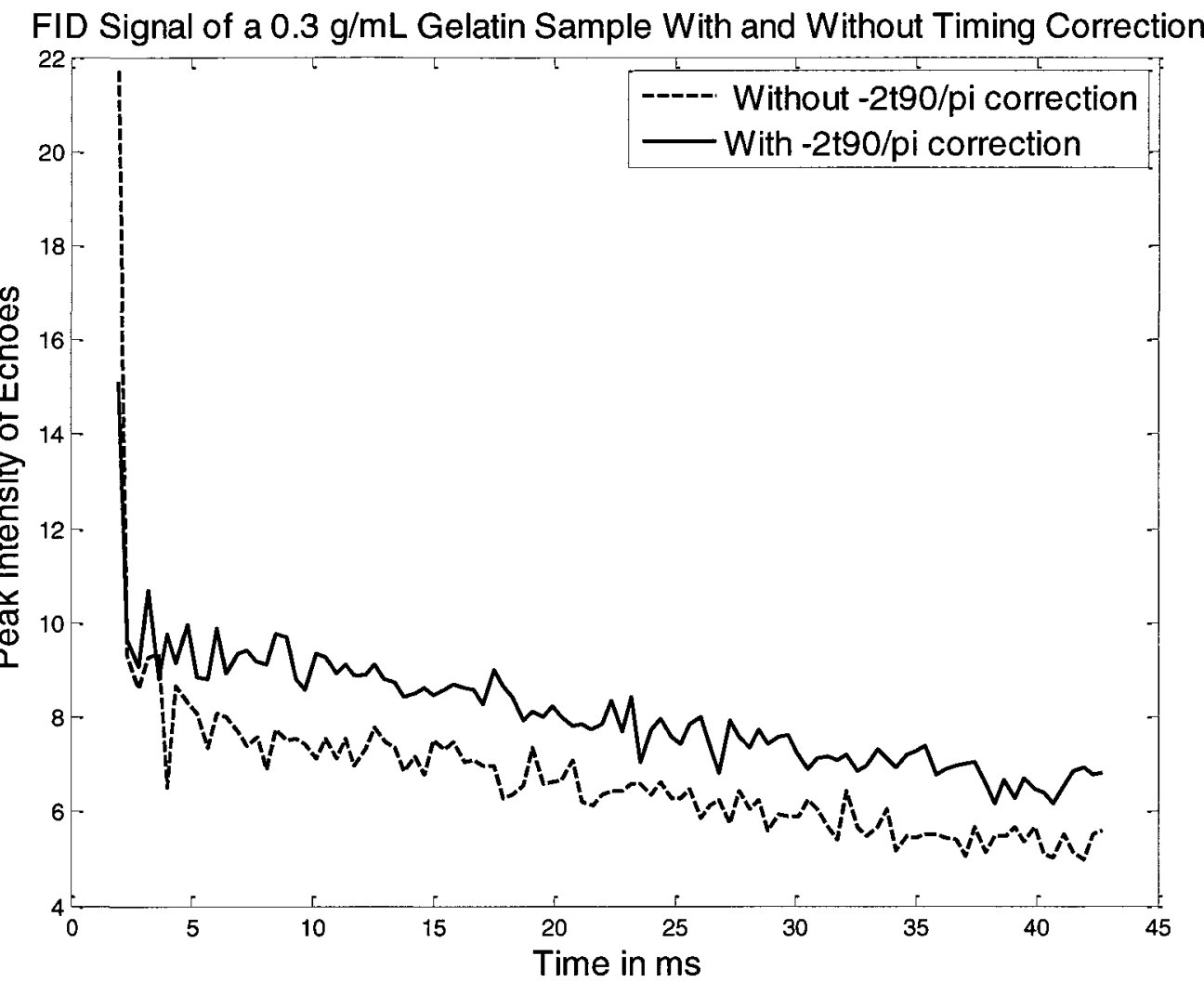

Figure 20 - Obtained peaks of echo train for a $0.3 \mathrm{~g} / \mathrm{mL}$ Gelatin Sample with and without the pulse spacing correction. It is observed that with the timing correction, the signal echoes have a higher signal intensity than with no correction. The scanning parameters were: 240 Scans/point, 80 Points per Echo, 0.1 Acquisition time, and 100 Echoes. 


\section{Simulation Environments and Experiments}

This chapter will describe experimental procedures and testing that was conducted for this thesis work.

\subsection{Phantom Development}

To evaluate the techniques developed in the previous chapters, it was essential to create several samples that mimic human tissue and that vary in elasticity. One way to create tissue mimicking samples is to use gelatin or agar [77]. Gelatin was chosen as the material to make the phantoms for this research in evaluating our system because it's stress and strain curve has less elastic hysteresis than agar [3]. This means that there is less energy dissipation to the environment as heat which will keep temperature more constant; in addition, the velocity during loading and unloading would remain similar, and that its stress and strain curve can be approximated by Hooke's law verifying our assumptions for velocity imaging. Ethylene glycol was chosen to be the solvent because it is more resistant to bacteria and has a much higher boiling point than water which can prolong the life of the sample [78]. The following section gives a detailed description of the procedure developed in creating a phantom from gelatin.

There are many different ways to create a gelatin sample in the literature. However, there is no standard for creating such a phantom. The key feature in creating these samples was to ensure that the five phantoms had different levels of elasticity and 
were similar in size. It has been verified in the literature that elasticity of the phantoms can be controlled by varying the gelatin concentration [3]. The equipment used for the creation of these phantoms were a hotplate magnetic stirrer, a magnetic stir rod, a fume hood, a beaker, a thermometer, a scientific balance with a precision of $0.0001 \mathrm{~g}$, aluminum muffin cup, gelatin powder (Gelatin Type A with a Bloom $\sim 225$ ), and $\sim 99 \%$ pure ethylene glycol from Sigma-Aldrich. The procedure below was followed to create the 5 gelatin samples:

1. Place beaker on the hotplate magnetic stirrer placed inside the fume hood.

2. Measure $40 \mathrm{ml}$ of ethylene glycol and pour it in the beaker.

3. Place the stir rod into the beaker and turn the stirrer on at medium stir rate.

4. Turn on the fume hood. Heat the ethylene glycol to $120^{\circ} \mathrm{C}$ using the thermocouple to keep track of the temperature.

5. Using the scientific balance, measure the following amounts of gelatin 9, 12, $15,18,21$ grams.

6. Once the ethylene glycol reaches $120^{\circ} \mathrm{C}$, slowly mix $1 / 10^{\text {th }}$ of the gelatin in to the liquid while gradually increasing the stir rate to high.

7. Repeat step 6 until the entire gelatin has been thoroughly dissolved in the ethylene glycol.

8. Once the solution is properly mixed turn off the hotplate and the stirrer.

9. The solution should be transparent with no residue floating inside. While still inside the fume hood slowly pour the mixture on to an aluminum muffin cup.

10. Let the gelatin mixture cool in the fume hood for about $30 \mathrm{~min}$ before moving it 
to a refrigerator to prevent inhaling fumes.

11. Repeat steps 2-10 until all desired gelatin concentrations have been made.

The resulting gelatin samples are shaped as a cone frustum with the base having a diameter of $6.3 \mathrm{~cm}$, the top having a diameter of $5.08 \mathrm{~cm}$ and a height of $1.27 \mathrm{~cm}$ (Fig. 21 ).

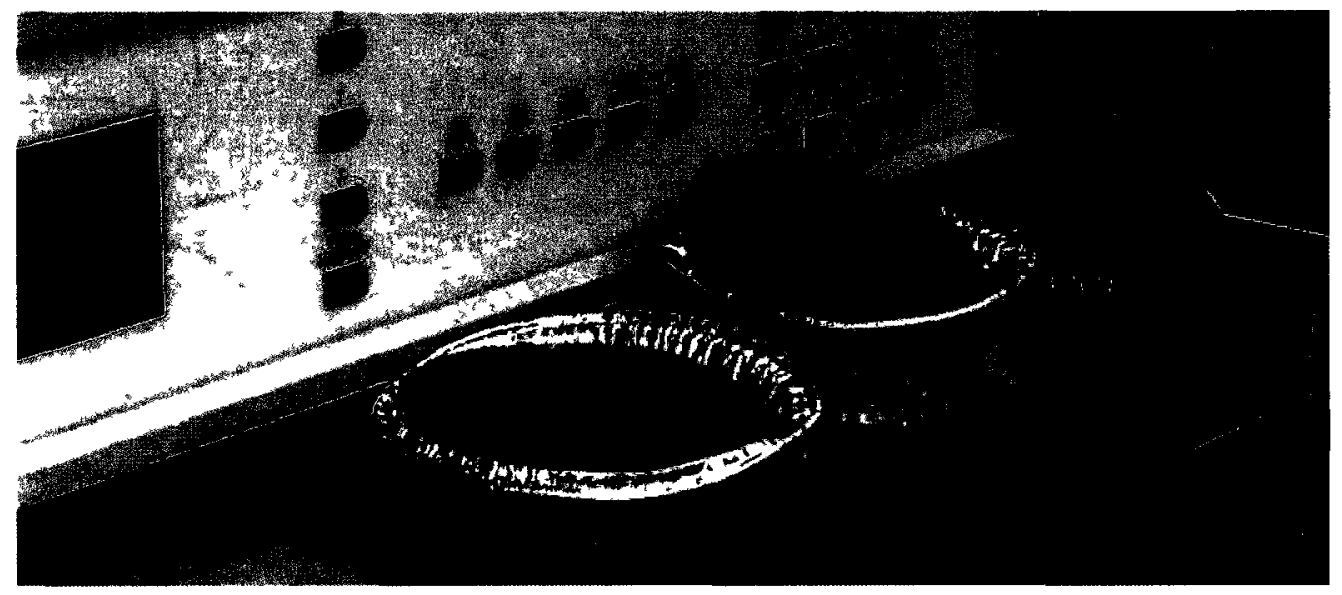

Figure 21 - Three of the five ethylene glycol gelatin samples created for this experiment.

To verify the stiffness of the samples we created, we performed an experiment in determining the samples Young's Modulus. From section 2.1, we can see that

$$
F=\frac{E A_{0}}{L_{0}} \Delta L=k \Delta L
$$

by rearranging Eq. 2.1. If we apply a known displacement and measure the force it takes to apply that displacement, we can plot a chart where the slope is proportional to $E$. To perform these measurements, the following equipment was used: a scale with a $0.1 \mathrm{~g}$ resolution, a thin cylindrical rod ( 0.25 inch diameter) and a Velmex position system (see Figs. 22, 23 and 24). The procedure for this experiment was as follows: 
1. Attach the cylindrical rod with a known area of $\mathrm{A}_{0}$ on the position system.

2. The sample to be measured is placed on a scale directly under the attached cylindrical rod.

3. Lower the cylindrical rod just so the entire surface area $A_{0}$ of the cylindrical rod is touching the surface of the sample.

4. Tare the scale so that the reading is $0 \mathrm{~g}$.

5. Using the positing system, compress the sample by the following distances: 0.025 , $0.254,0.318,0.419,0.559,0.667,0.8,0.902,1.118$, and $1.181 \mathrm{~mm}$.

6. While compressing through each distance, record the weight displayed as soon as the reading is stable. Immediately, start compressing the sample to the next distance.

7. Repeat step 5 and 6 until all data has been gathered.

The data was then analyzed to obtain different $k$. To obtain Young's modulus from $k$, we assume that the cross-sectional area of the force applied on the sample is very small compared to the sample. This way we can say that $A_{0}$ is just the cross sectional area of the cylindrical rod and $\mathrm{L}_{0}$ is the height of the sample. The equation (5.1) is valid for this experiment because the displacement applied on to the sample is so small that there is negligible "craters" that occur on the surface of the sample under compression. The following results are shown in Figs. 25 and 26. 


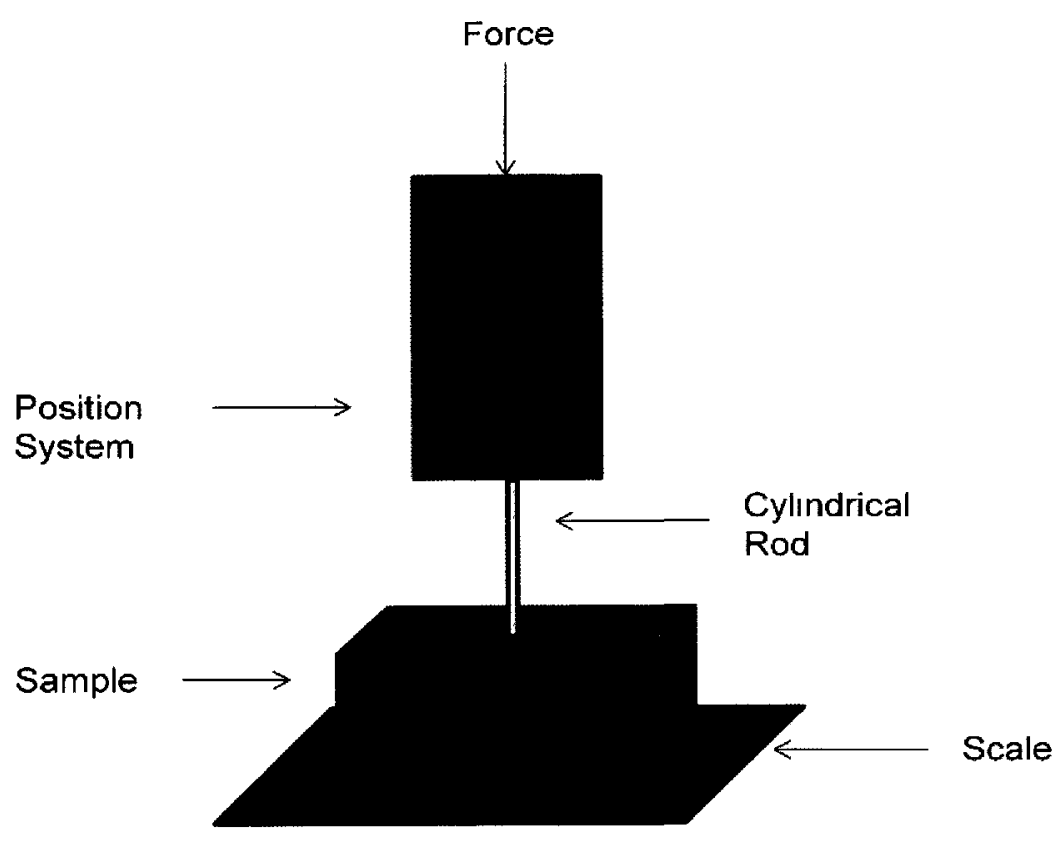

Figure 22 - A Block Diagram for the setup in measuring Young's modulus

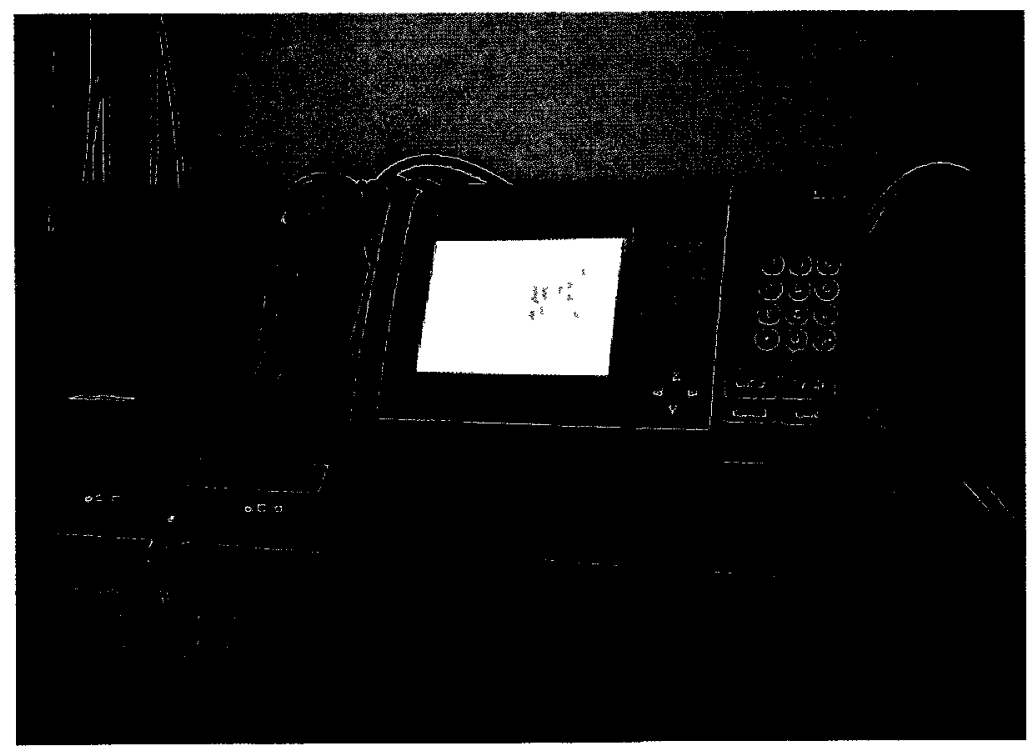

Figure 23 - VXM step motor and display. The display shows the displacement on any axis in mm or in inches. 


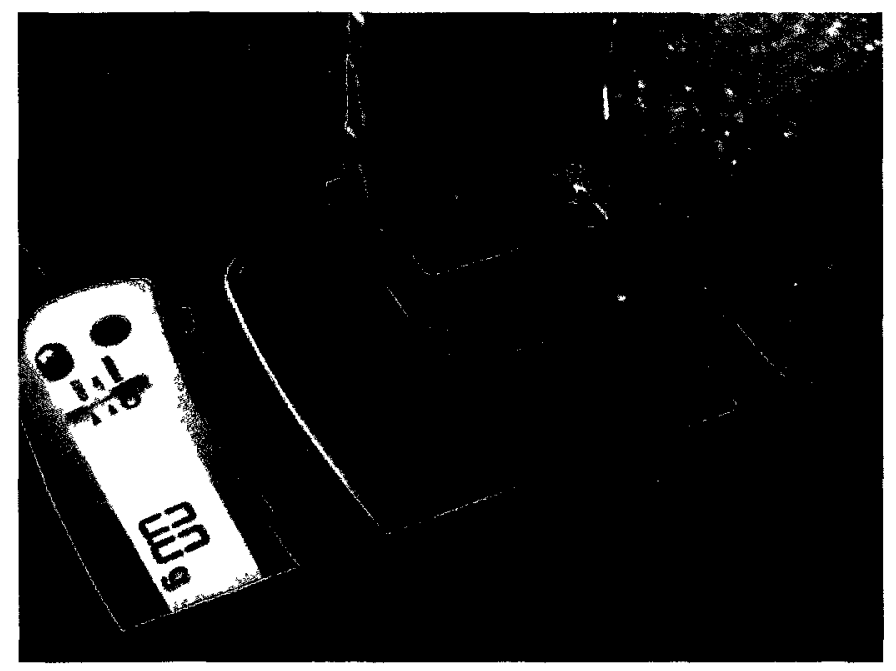

Figure 24-Gram scale with an ethylene glycol sample. A small cylindrical rod is mounted underneath the arm and is slightly pressing on the sample. The scale is set to zero at this position.

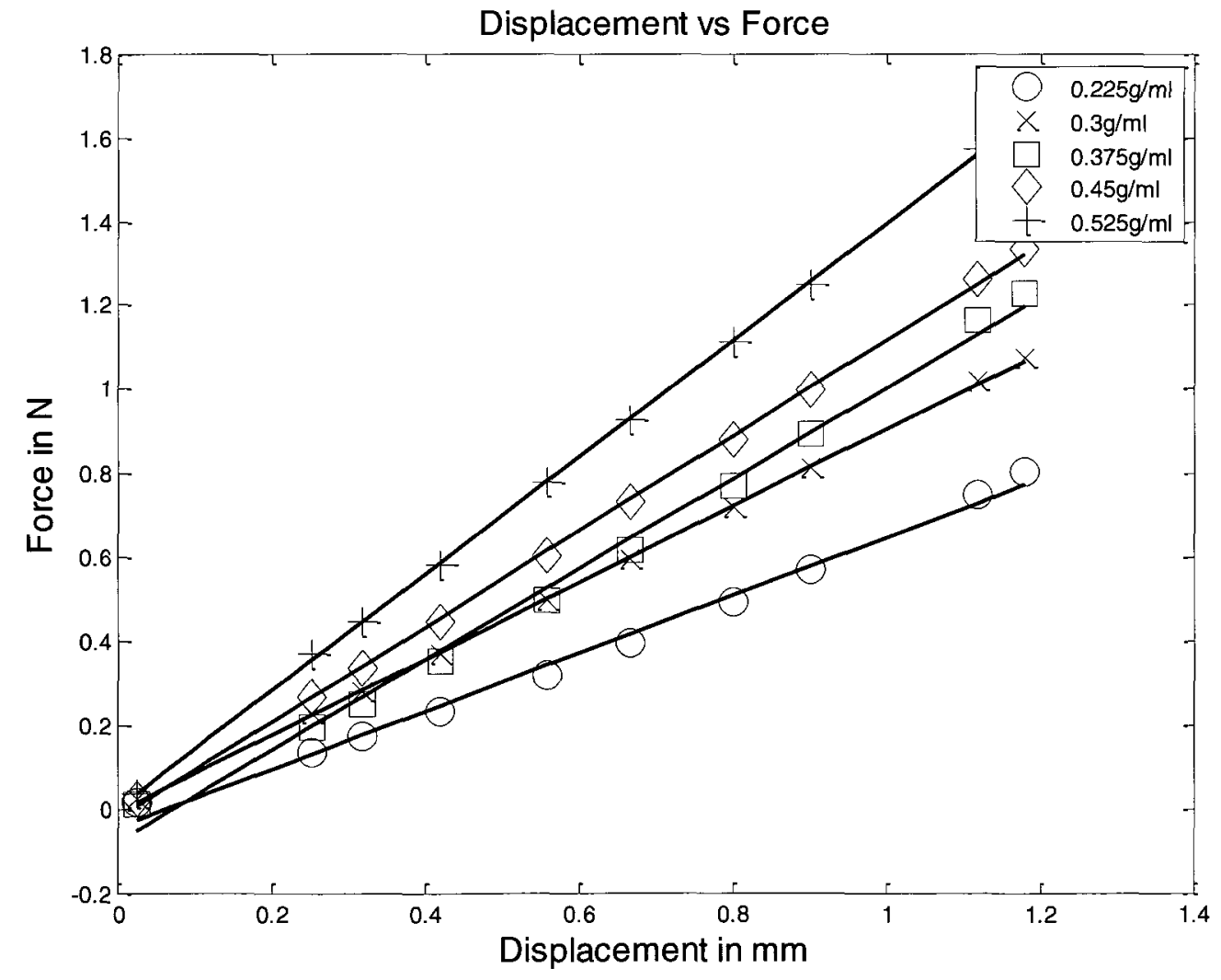

Figure 25 - Displacement vs Force graph for the created gelatin samples. 


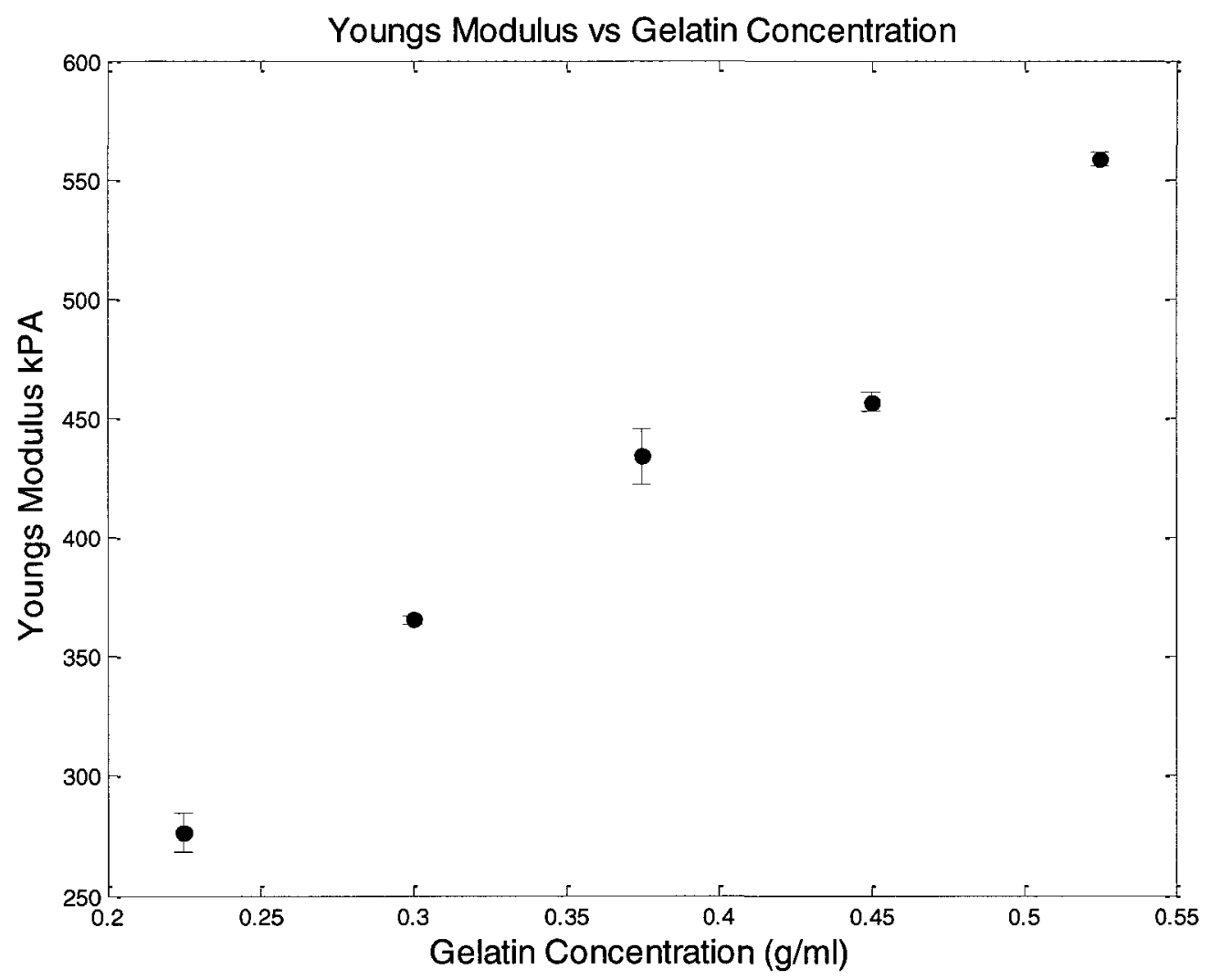

Figure 26 - Young's Modulus vs Gelatin Concentration plot with an error bar of +/1 standard deviation (eq 5.3). It shows that with increasing gelatin concentration, the Young's Modulus also increases. The calculated Young's modulus for the created samples are $276,365,434,457,559 \mathrm{kPa}$.

These gelatins are viscoelastic material which experiences hysteresis. However, as mentioned before, the hysteresis in these materials is small and was ignored for simplicity in calculating the Young's modulus. The results obtained in this experiment are in general agreement with the experiments conducted by Hall et al. on the Young's modulus of water based gelatin samples [3]. 


\subsection{Experimental Setup}

The following section will discuss in detail the experiments and work that were conducted for this thesis. The first experiment conducted was to find the optimal repetition time for the experiments given our samples. The second experiment deals with minimizing the distance between the external vibration source and the magnet. The third experiment deals with preliminary motion measurements using the CPMG pulse sequence. The final experiment deals with elasticity estimation.

\subsubsection{Optimizing Repetition Time}

The first experiment performed is to find a favorable repetition time between successive scans. The ideal repetition time between successive scans to be as small as possible while also ensuring that SNR remains high. If a subsequent scan is performed before $5 * \mathrm{~T} 1$, signal intensity will be much less and will greatly lower the signal-to-noise ratio. To achieve the highest SNR at the minimal amount of time, we performed the experiment on the softest material since typically the material with the highest $\mathrm{T} 1$ will be from the material with highest mobility, or a more elastic sample. The result of the experiment is shown in Fig. 27. It is observed at TR less than $800 \mathrm{~ms}$, significant loss of signal intensity is observed. At TR between $800-1000 \mathrm{~ms}$, a small improvement in signal intensity can be observed. After $1000 \mathrm{~ms}$, no statistically significant improvement signal intensity is observed. From this data, we chose TR to be $1000 \mathrm{~ms}$ for all subsequent scans. 


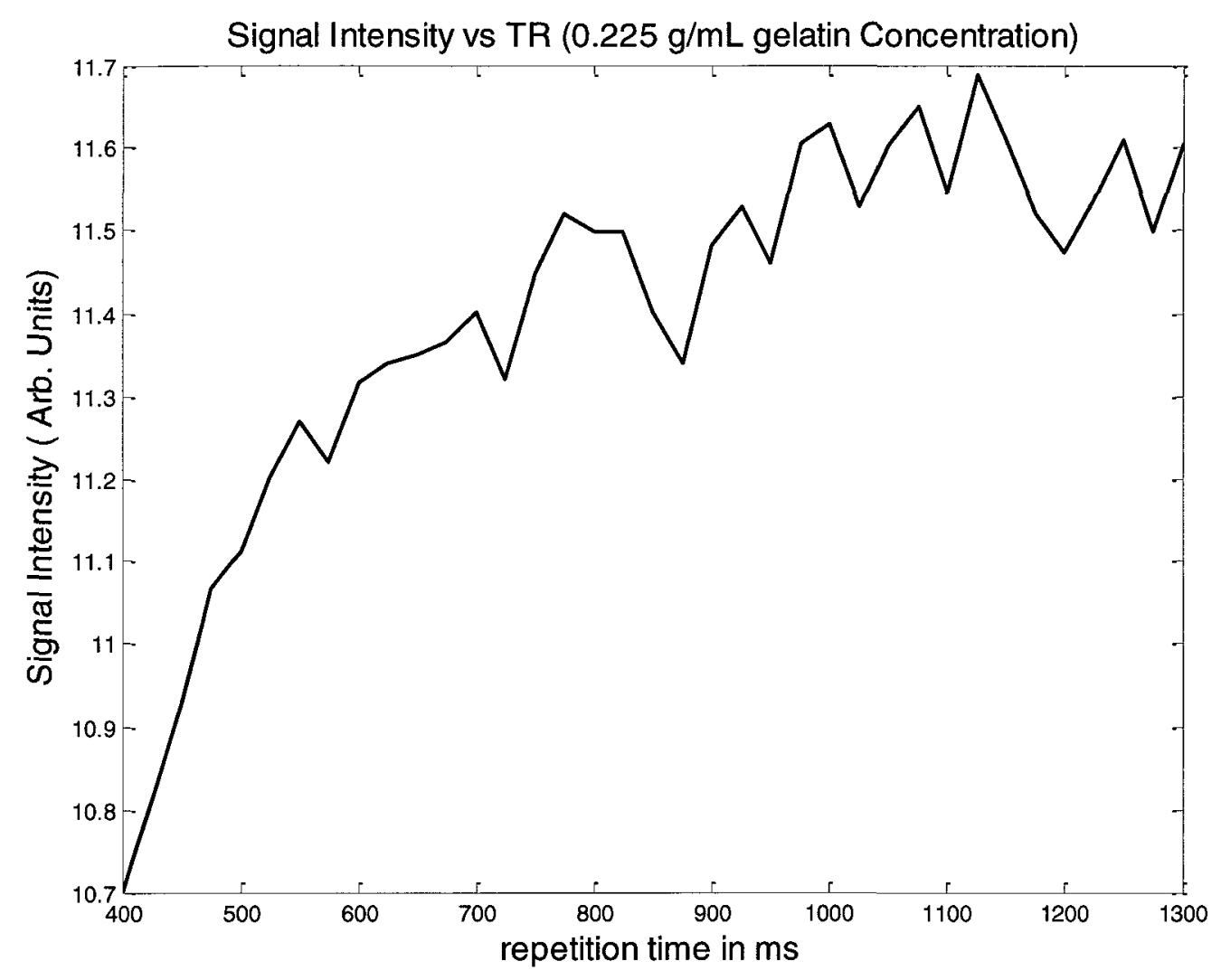

Figure 27 - Average Signal Intensity at each TR. We can observe that at approximately $1000 \mathrm{~ms}$, the signal intensity does not significantly improve anymore. The scanning parameters were: 400 Scans/point, 80 Points per Echo, 0.3 Acquisition time, and 101 Echoes.

\subsubsection{Massager Placement}

The next experiment we did was to determine the distance at which the massagers will have no effect on the acquired MRI signal. The massager cannot be placed near the magnet as the massager contains a motor which will create a magnetic field which could potentially introduce noise into the measurements. The massager would then instead 
need to be on a propagating medium to transfer mechanical waves to the sample. However, the propagating medium needs to be as short as possible as attenuation of the mechanical waves are proportional to the length of the propagating medium. To find the minimum distance where the massager can be placed, we performed the following procedure:

1. Attach the massager on the position system.

2. Place a sample on top of the RF coil.

3. Perform an MRI scan with moving the massager away from the magnet at increments of $0.635 \mathrm{~cm}$ along the z-axis at $\tau=0.2 \mathrm{~ms}$.

4. The obtained MRI signal is then processed and plotted using average signal amplitude vs distance from the surface of the magnet.

This procedure was performed on both massagers. The results are shown in Fig. 28 and 29 for both the Conair massager and the Dr. Scholl's massager respectively. 
Signal Intenisty vs Massager Distance from the Surface of the Magnet (Conair Massager)

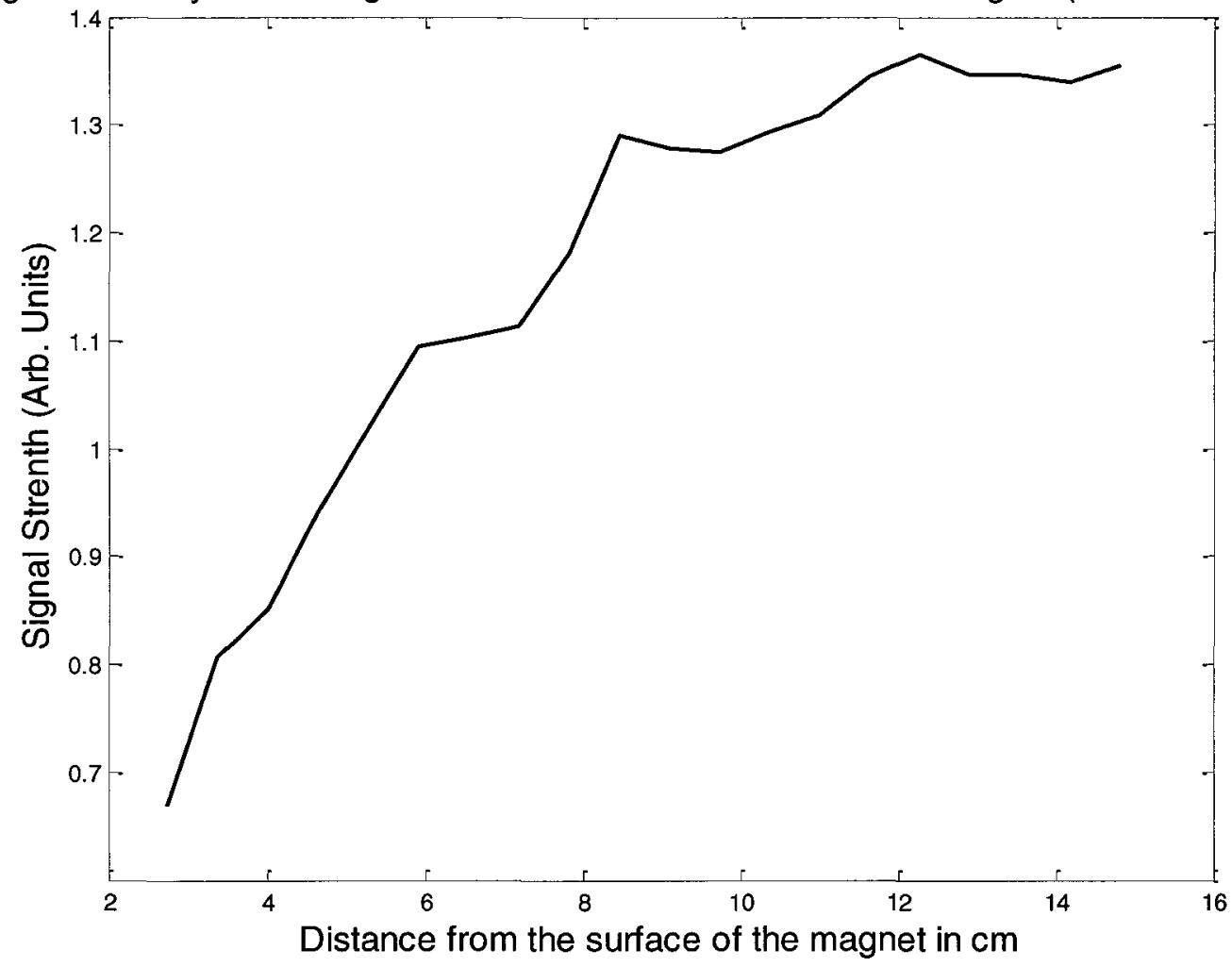

Figure 28 - Signal Intensity vs Massager Distance from the Surface of the Magnet. The massager used for this data is the Conair Massager. We can see that when the massager is close to the magnet, the signal intensity is much lower than when the massager is placed farther away from the magnet. The scanning parameters were: 1000 Scans/point, 80 Points per Echo, 0.3 Acquisition time, and 200 Echoes. 
Signal Intenisty vs Massager Distance from the Magnet Surface (Dr. Scholls Massager)

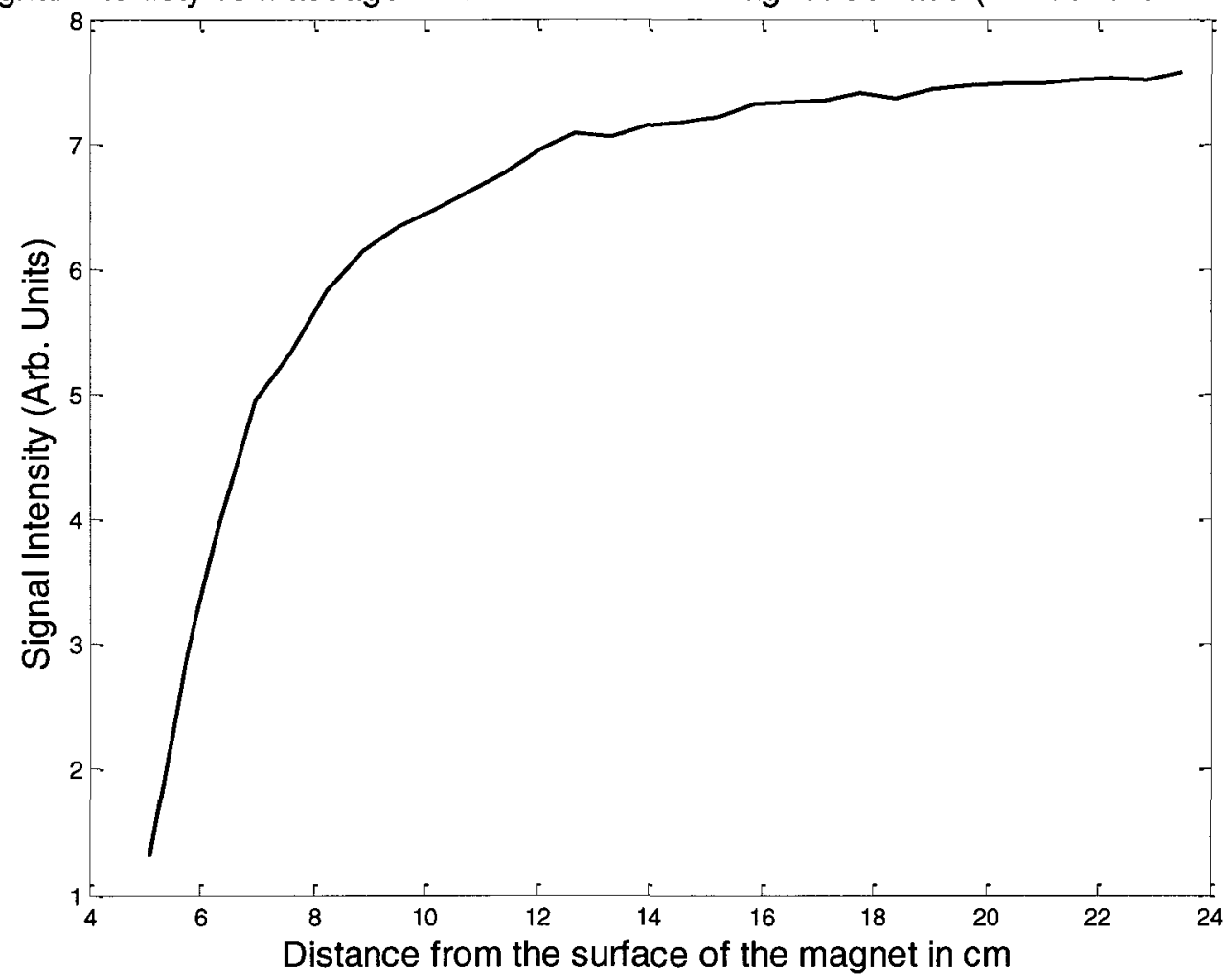

Figure 29 - Signal Intensity vs Massager Distance from the Surface of the Magnet.

The massager used for this data is the Dr. Scholl's Massager. The scanning parameters were: 128 Scans/point, 80 Points per Echo, 0.1 Acquisition time, and 300

Echoes.

Inspecting both graphs, significant improvement in SNR is observed for the first $12.7 \mathrm{~cm}$ and significantly less improvement after $12.7 \mathrm{~cm}$. Therefore, we decided that the height of the propagating medium be $12.7 \mathrm{~cm}$. The material chosen for the propagating medium is wood. Wood does not affect the magnetic field and has a T2 much smaller than the material sample used in this experiment. 


\subsubsection{Preliminary Motion Experiments - Conair Massager}

To validate the theory discussed in chapter 4 , preliminary experiments were conducted to see if motion can be detected. For this setup, the vibration source used is the Conair massager with the wood block stack at $12.7 \mathrm{~cm}$ high. The block diagram for this setup is shown in Fig. 30 and the experiment setup is shown in Fig. 31. The following subsections will use this setup.

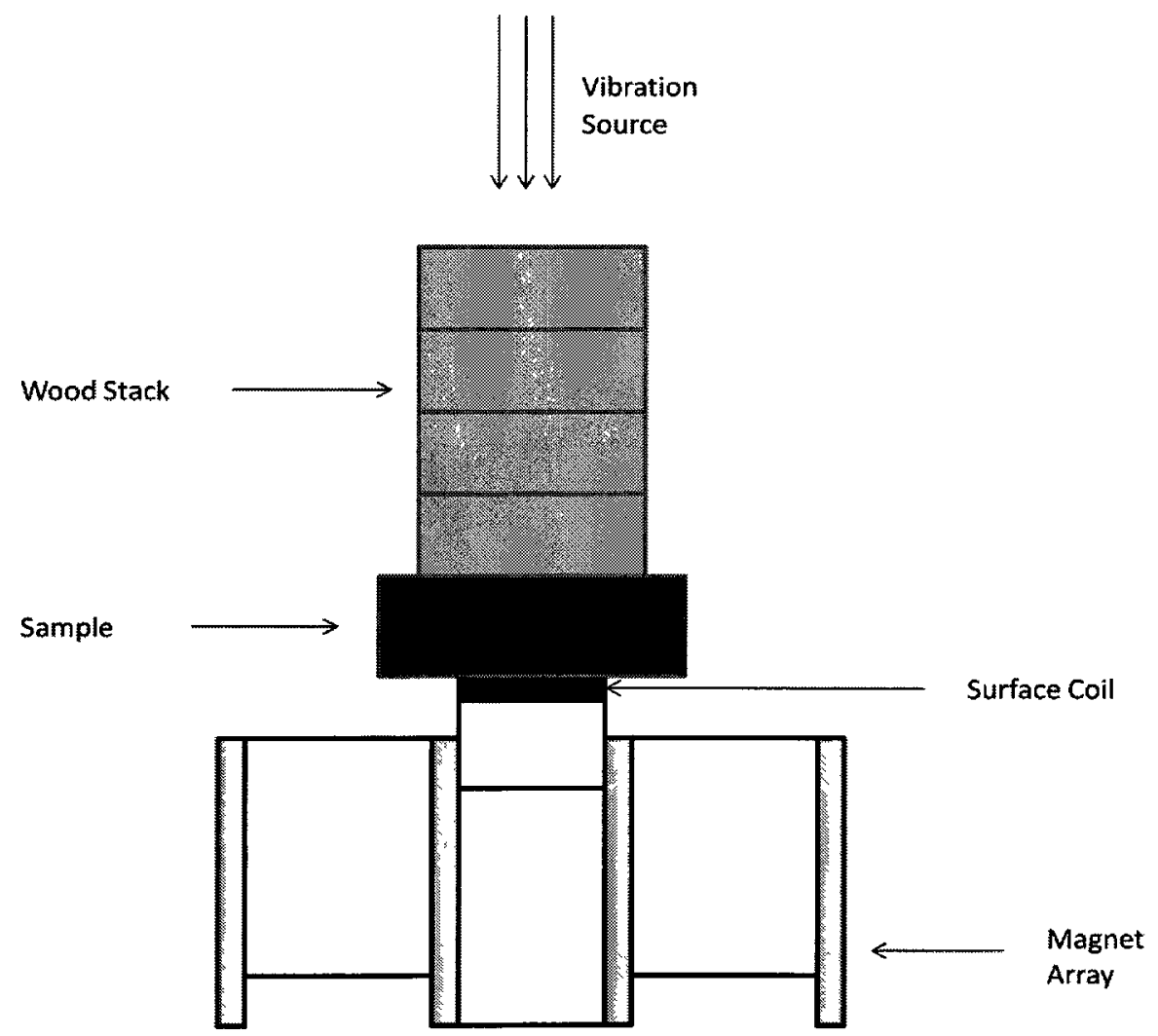

Figure 30 - Slice View block diagram of the first experimental setup for preliminary experiments. 


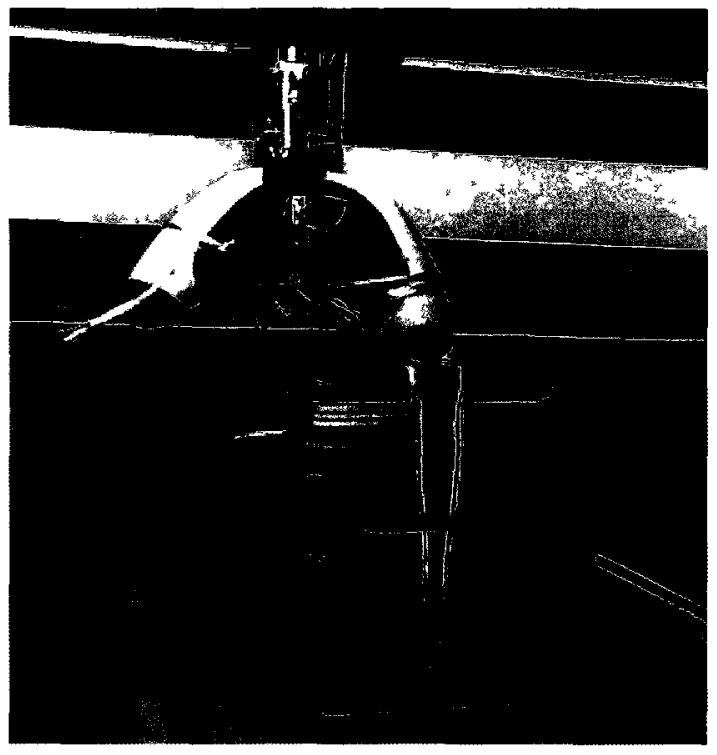

Figure 31 - Experiment setup with Conair Massager.

\subsubsection{Preliminary MRI Measurements and Results}

The first of these experiments was to determine if motion causes signal attenuation in the echoes and not other external sources. CPMG pulse sequence was used with a silicon rubber sample. This silicon rubber is much stiffer compared to the created samples. As mentioned in section 5.1, applying a CPMG pulse sequence at any $\tau$ should have no effect on the received signal intensity. This means that the $\mathrm{T} 2$ decay of the sample should be the same at any $\tau$ under no motion. Scanning parameters are: 1000 Scans, $0.3 \mathrm{~ms} \mathrm{acq,} 80$ data per echo and 600 echoes at $\tau=0.2,0.3,0.4,0.5,1,1.5 \mathrm{~ms}$. The results are shown in Figs. 32, 33, 34, 35 and 36. To calculate the T2 constant from the acquired signal, we can modify Eq. 2.7 in terms of $\tau$

$$
\ln \left(M_{x y}\right)=-\frac{1}{T 2} \tau+\ln \left(M_{0}\right)
$$

It is shown that the $\ln$ of acquired signal is inversely proportional to $\mathrm{T} 2$. 

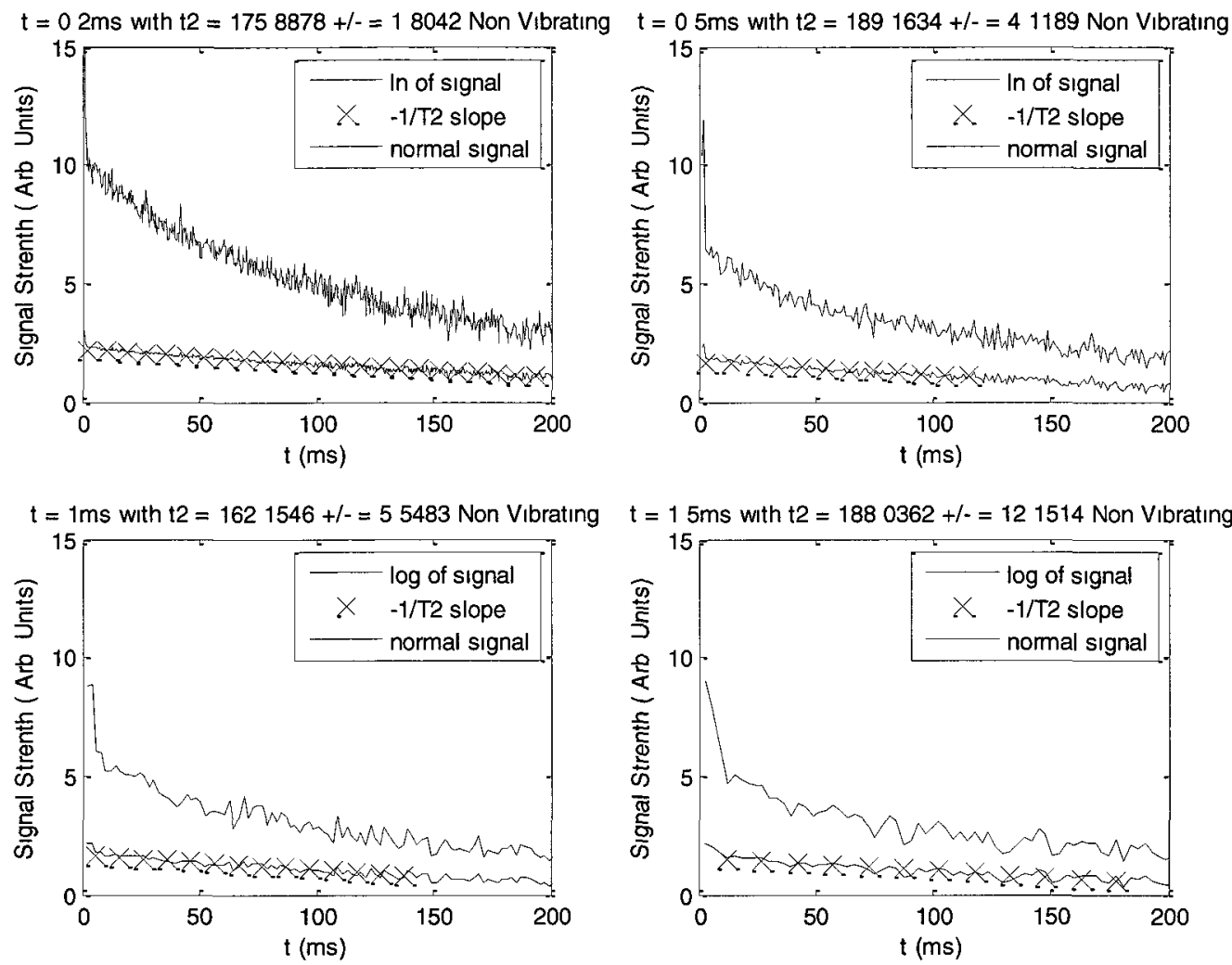

Figure 32 - Measured signal Intensity of silicon rubber under no motion at various

t. T2 is measured from 0 until $200 \mathrm{~ms}$ or until the signal-to-noise ratio is too low. 

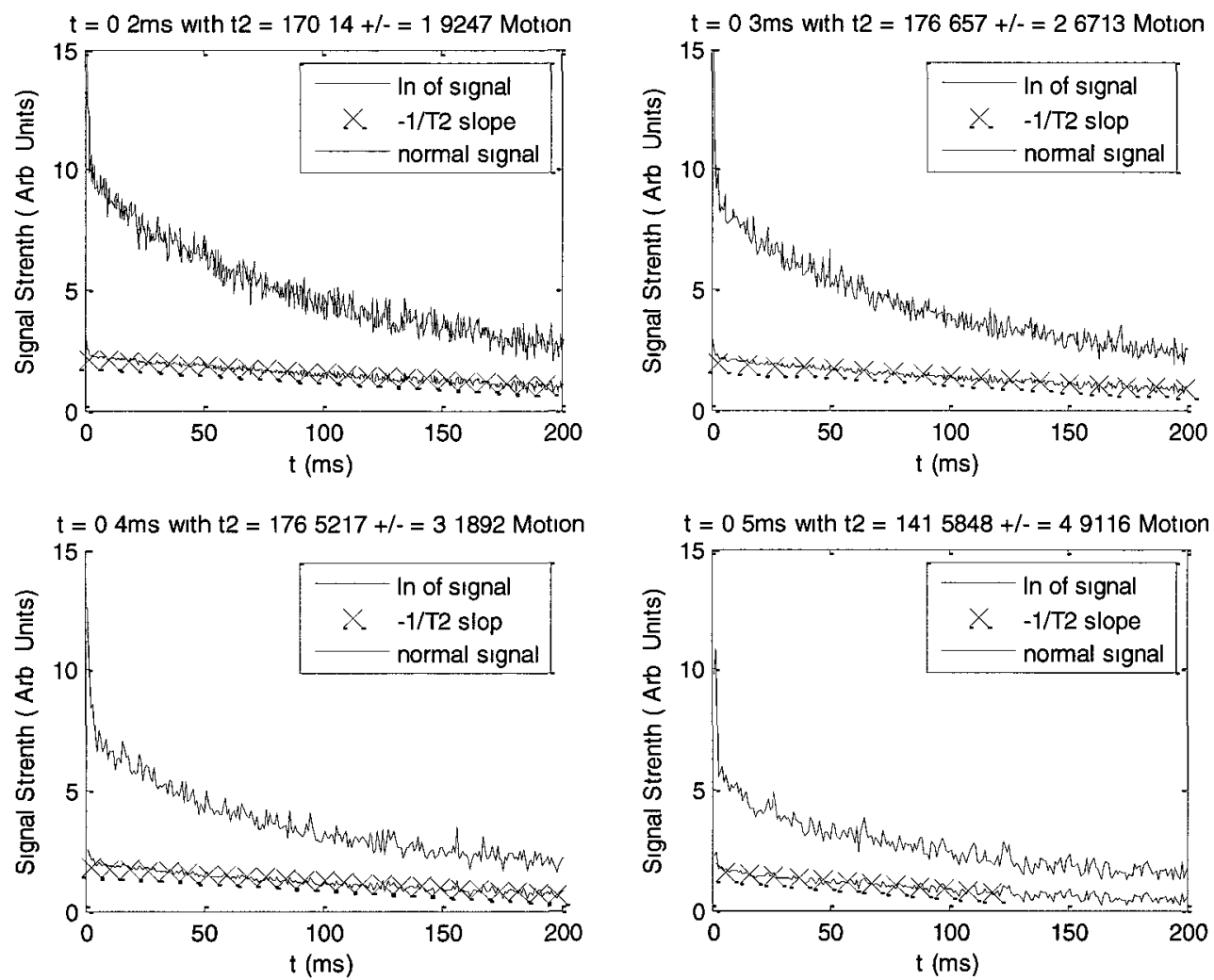

Figure 33 - Measured signal Intensity for silicon rubber under motion at various $\tau$ $=0.2,0.3,0.4,0.5$. $T 2$ is measured from 0 until $200 \mathrm{~ms}$ or until the signal-to-noise ratio is too low. 

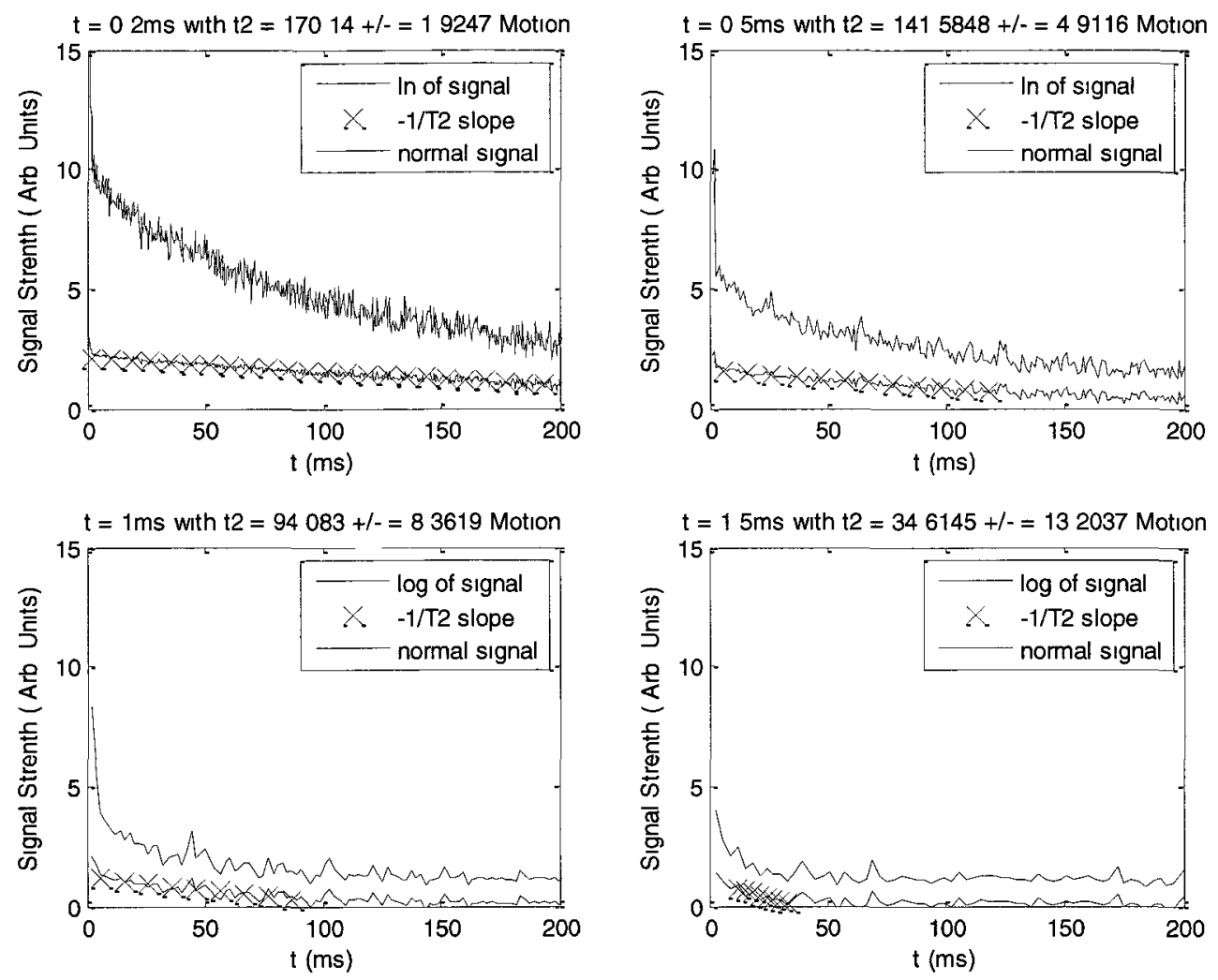

Figure 34 - Measured signal Intensity for silicon rubber under motion at various $\tau$.

T2 is measured from 0 until $200 \mathrm{~ms}$ or until the signal-to-noise ratio is too low. 


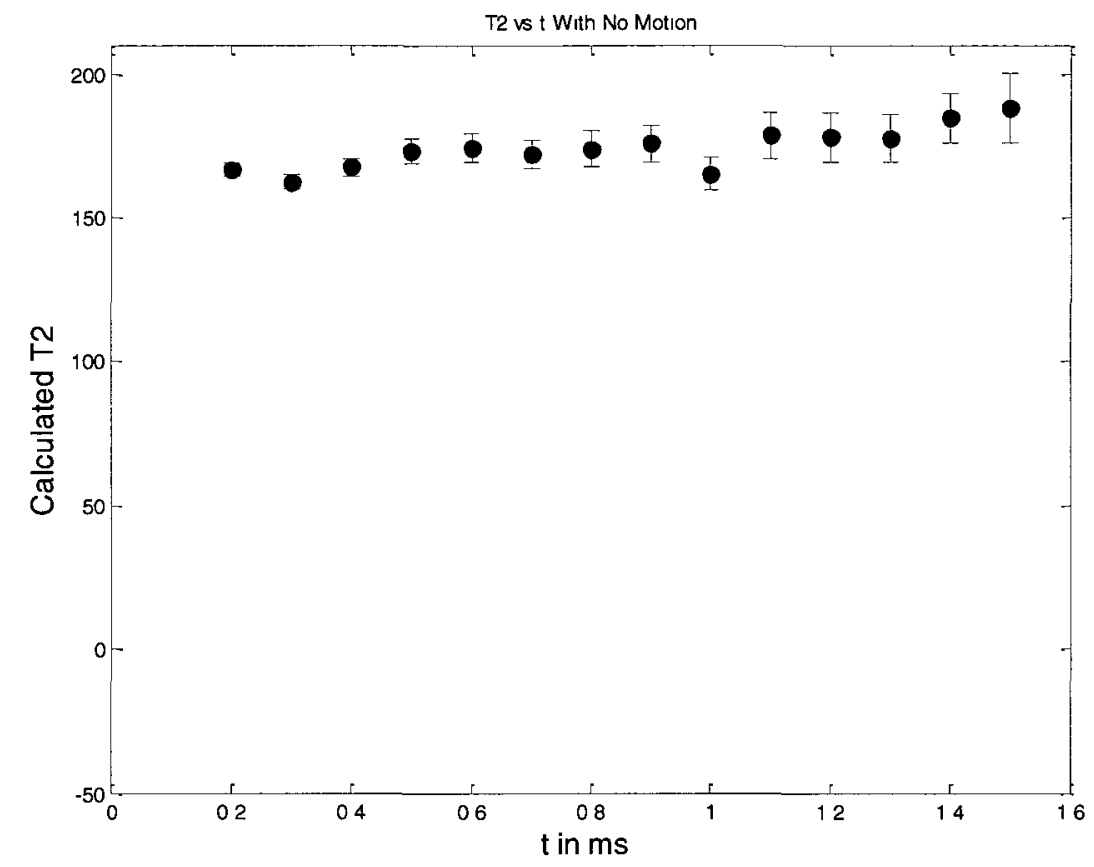

Figure 35- T2 vs $\tau$ plot sample under no motion. It shows that $\mathbf{T} 2$ does not change statistically as $\tau$ increases.

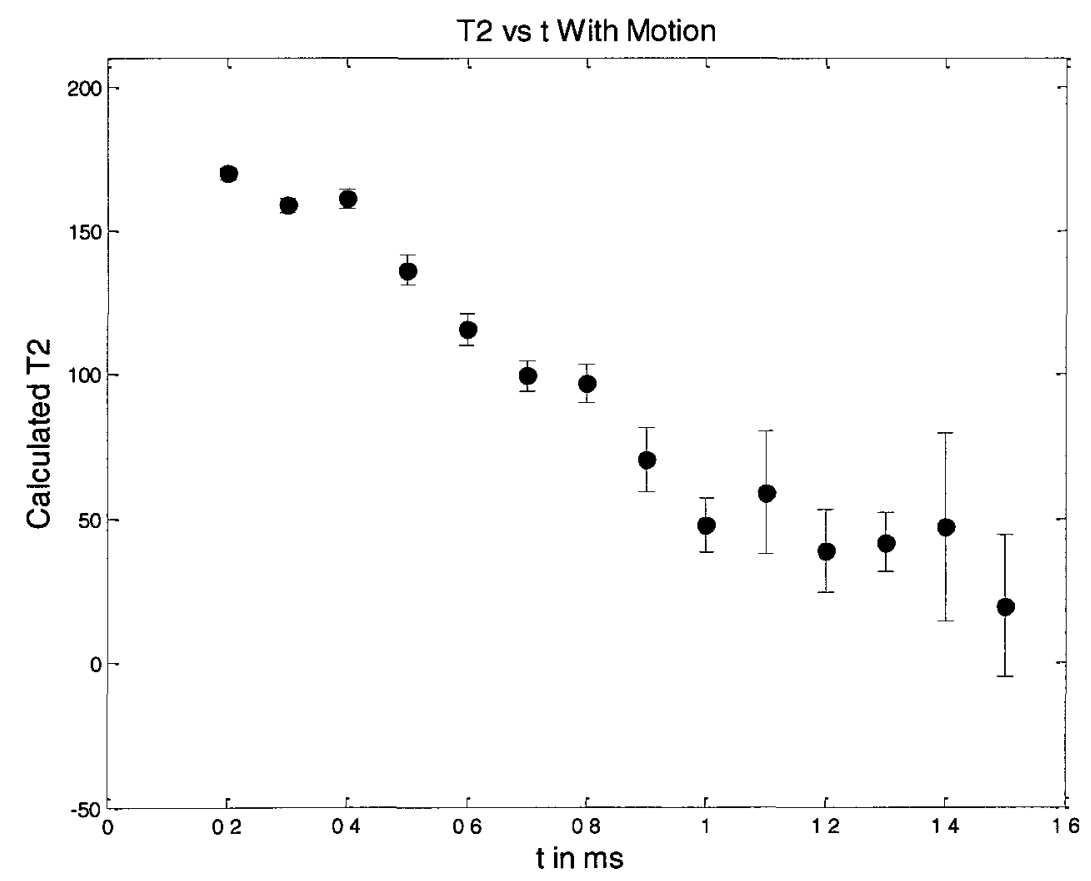

Figure $36-T 2$ vs $\tau$ plot sample under motion. It shows that T2 starts to decrease at $\tau$ greater than $0.4 \mathrm{~ms}$. At $\tau>1 \mathrm{~ms}$, the remaining signal is mostly noise. 


\subsubsection{Preliminary Measurements - Discussion}

The results obtained when the sample is under no oscillatory force shows that $\mathrm{T} 2$ constant remains statistically the same. Under motion, T2 constant decreases with increasing $\tau$. The standard deviation of error for the best fit line was calculated using $t-$ statistics [79]

$$
s t d=\sqrt{\frac{\frac{1}{n-2} \sum_{i=1}^{n}\left(y_{i}-F\left(x_{i}\right)\right)^{2}}{\sum_{i=1}^{n}\left(x_{i}-\bar{x}\right)^{2}}}
$$

where $n$ is the number of data points, $x_{i}$ and $y_{i}$ are $i^{i h}$ data point of the original data set, $\vec{x}$ is the mean of $\mathrm{x}$ and $F\left(x_{i}\right)$ is the best fit line. This is the desired results as it agrees with our assumptions from before that at low $\tau$ (less than $0.5 \mathrm{~ms}$ ), minimal phase dispersions occur due to very fast refocusing, while at high $\tau$ high signal attenuation occurs due to phase dispersion. A key observation is that the first five echoes of the MRI signal contains an abnormally high intensity for all scans. This can be due to hardware error such as the RF coil ringing or pulse sequence timing error between the first $90^{\circ}$ and $180^{\circ}$ pulses. The experiment time to obtain one plot took approximately $20 \mathrm{~min}$. To speed up the scan time for future experiments conducted in this work, the number of scans was lowered to 400 . It is observed that this number of scan can still provide a high enough SNR at high $\tau$ when the sample is under vibration. To further speed up the repetition time between scans, the SGSE-CPMG pulse sequence is used. 


\subsubsection{Preliminary Measurements - Acquisition Time Optimization}

In an ideal MRI signal acquisition, only one data point per echo is needed if we know for certain that the obtained data point will land at the peak of the echo. To give us an error margin to locate the peak of this echo, 80 data points per echo was chosen. The sampling window of the $a c q$, determines the length of time to sample the echo. In the initial MRI signal measurements, $a c q$ was defaulted to $0.3 \mathrm{~ms}$ where it shows that many of the data points landing outside the main echo. A simple experiment was then conducted with varying $a c q$ from 0.1 to $0.3 \mathrm{~ms}$ with increments of $0.1 \mathrm{~ms}$ to find a better value. The results are shown in Fig. 37. The results show that at $a c q=0.1 \mathrm{~ms}$, almost all the 80 data points land on the peak of the echo. Furthermore, at lower acq, less noise are obtained in the first echo. 


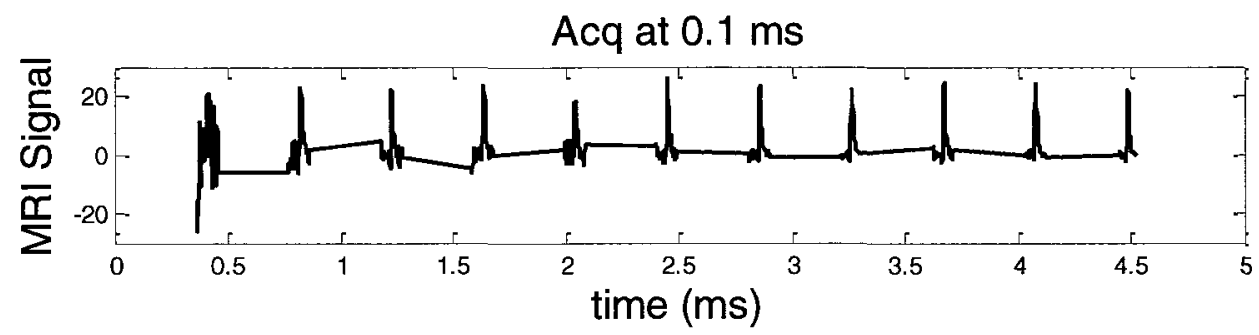

Acq at $0.2 \mathrm{~ms}$

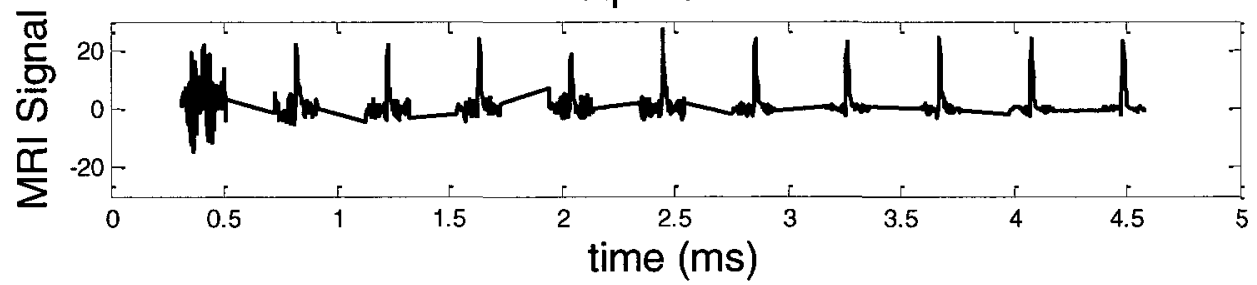

Acq at $0.3 \mathrm{~ms}$

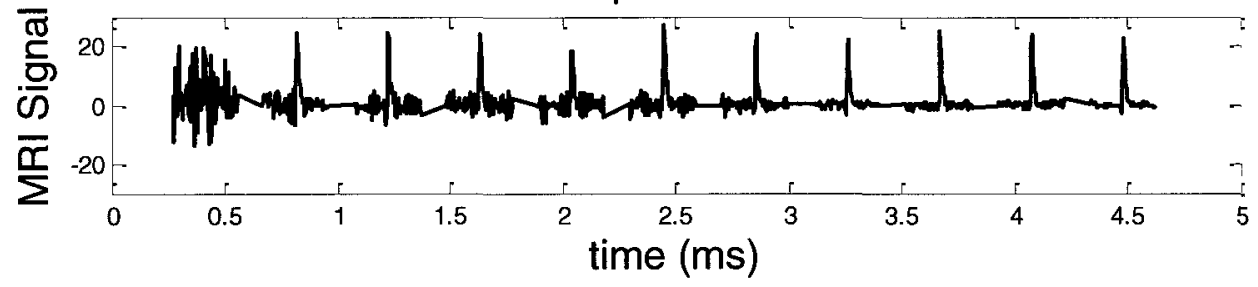

Figure 37 - Plot of 10 echoes with varying $a c q$ at 480 scans on a $0.225 \mathrm{~g} / \mathrm{mL}$ ethylene glycol sample.

\subsubsection{P-space Measurements and Results}

In the previous experiment, it was verified that the measured signal decays with increasing $\tau$ under motion. The next step is to obtain $\boldsymbol{P}$-space measurements from the measured signal to verify that in motion can be observed in $\boldsymbol{P}$-space. To obtain points in $\boldsymbol{P}$-space, the echoes of the measured signal are ensemble averaged to produce a single data point in $\boldsymbol{P}$-space. The scanning parameters for the pulse sequence are as follows: $\boldsymbol{p}$ is sampled in $\tau^{2}$ from 0.04 to 3.96 with an increment of $0.04,400$ scans, $0.1 \mathrm{~ms} a c q, 601$ 
echoes and at 80 data points per echo. The results for the silicon rubber are shown in Figs. 38 and 39 and for a real stiff ethylene glycol gel are shown in Figs. 40 and 41.
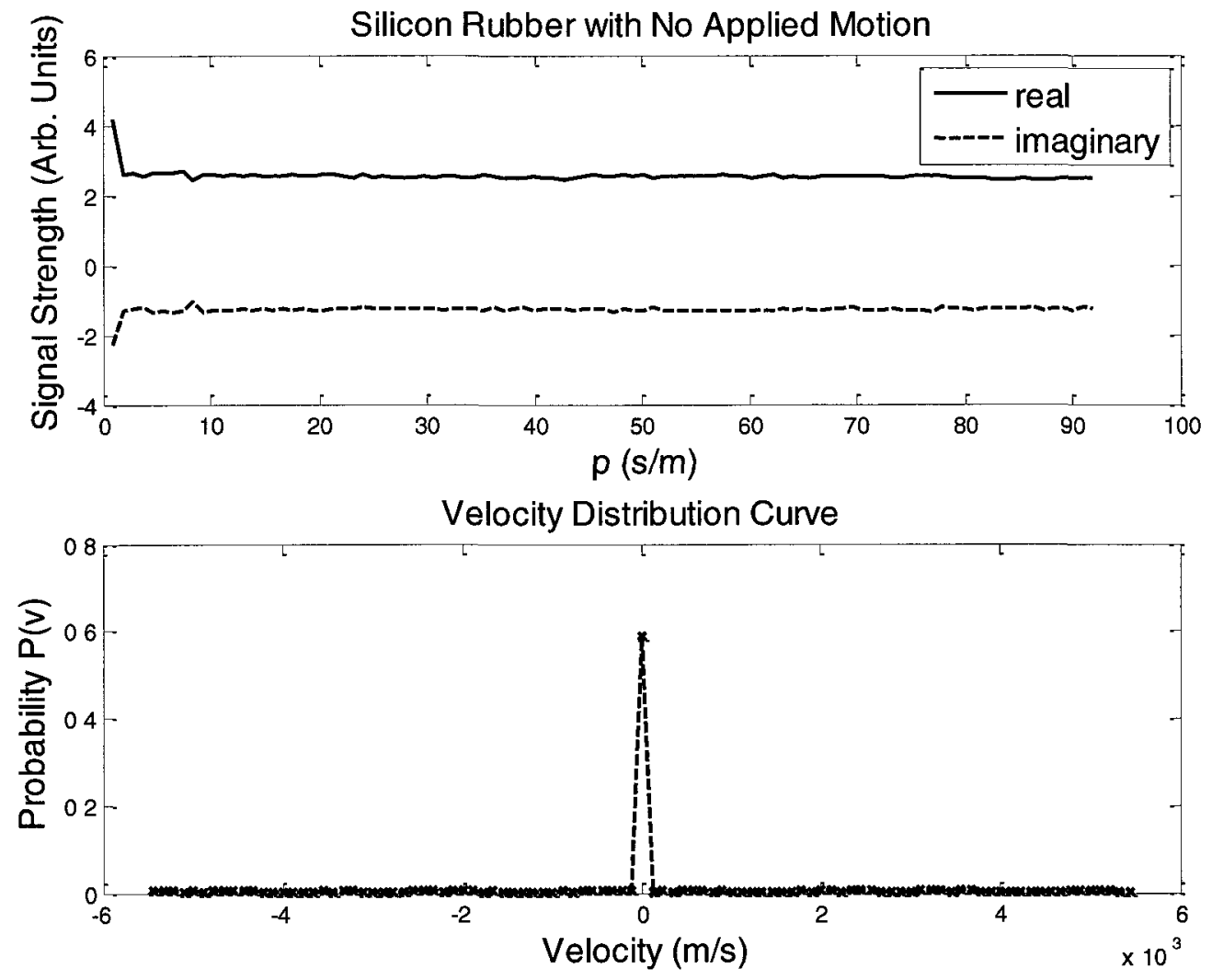

Figure 38 - Resulting P-space plot on silicon rubber with no applied motion. The velocity distribution curve resembles a delta function centered at 0 velocity. 


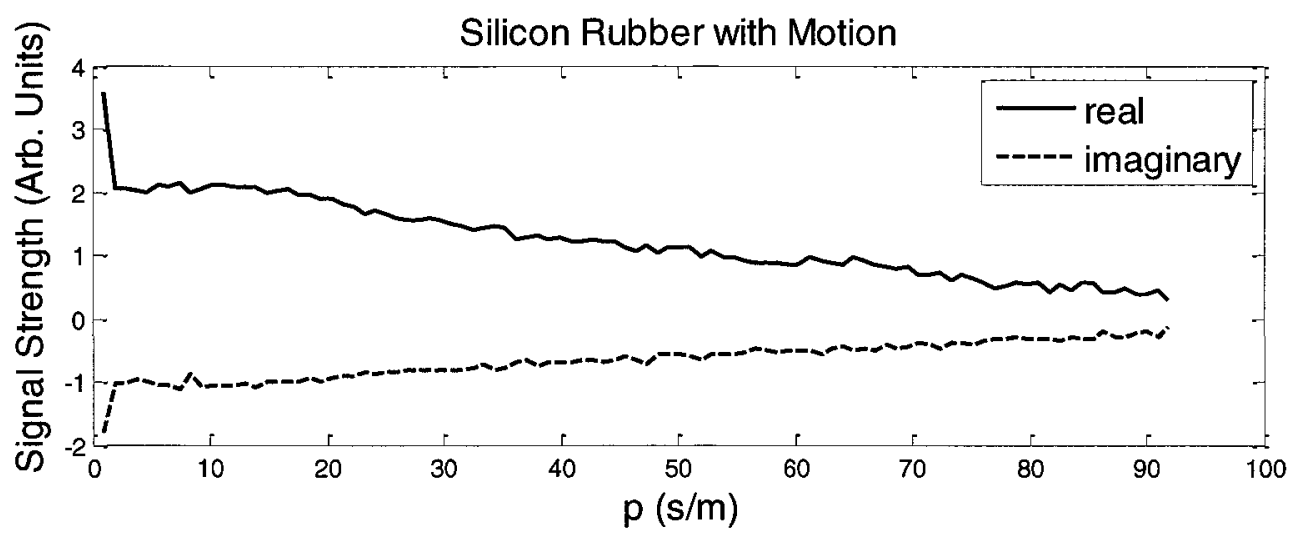

Velocity Distribution Curve

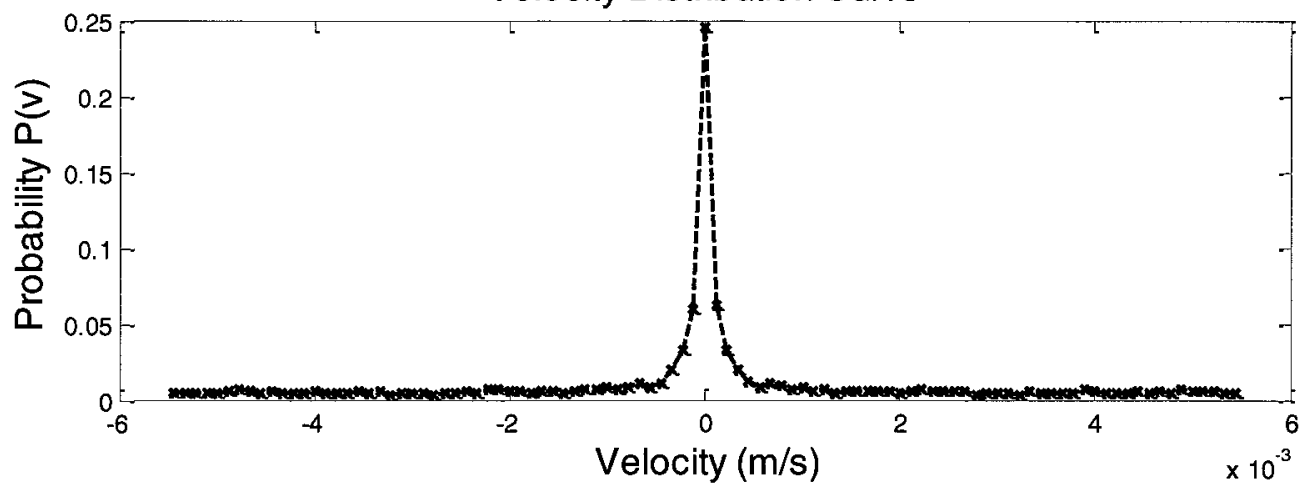

Figure 39 - Resulting P-space plot for Silicon Rubber with Applied Motion. The velocity distribution curve has a shaped distribution implying motion is measured. 

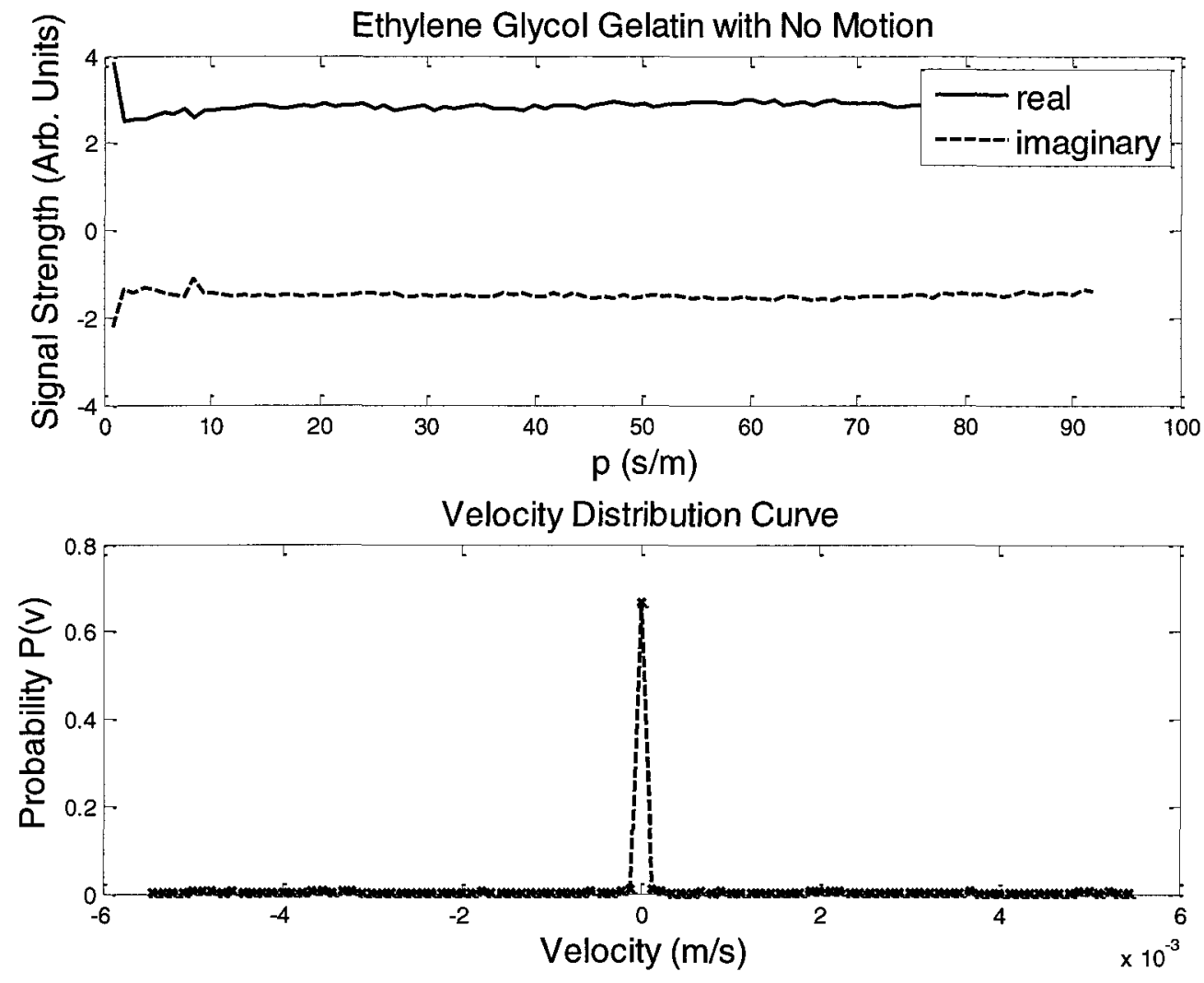

Figure 40 - Resulting P-space plot for Ethylene Glycol with no Applied Motion.

The velocity distribution curve resembles a delta function centered at 0 velocity. 

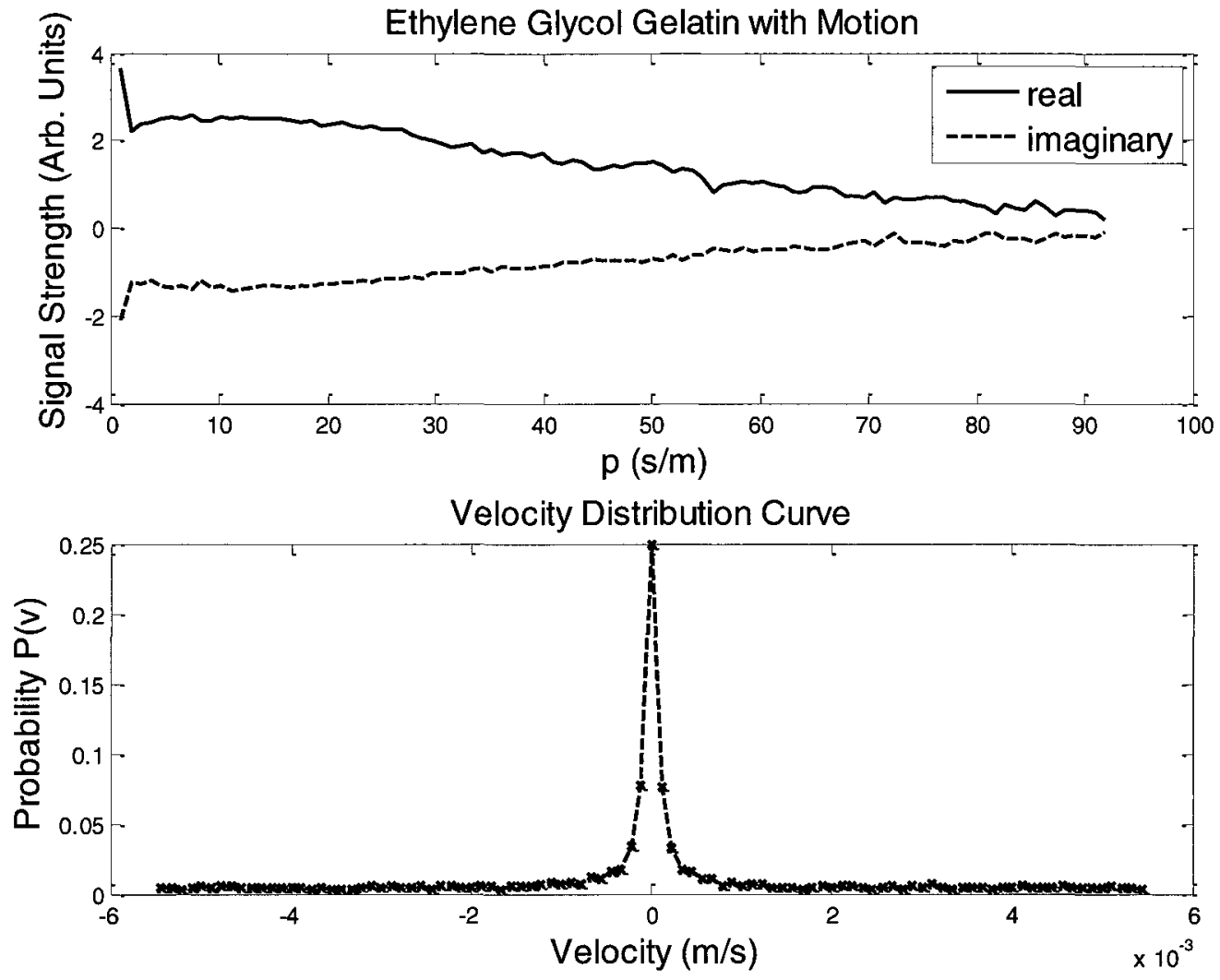

Figure 41 - Resulting P-space plot for Silicon Rubber with Applied Motion. The velocity distribution curve has a bell shape distribution implying that motion is measured.

It is observed that signal intensity decreases in $\boldsymbol{p}$ if motion is applied on the sample. Under no motion, it can be seen that the velocity probability distribution curve resembles a delta function with a mean at $0 \mathrm{~m} / \mathrm{s}$. These results agree with the theory discuss in section 4.1 . 


\subsubsection{Motion Experiments with Modified Setup - Dr. Scholl's Massager}

The previous experimental setup needed improvements because it was observed that the created ethylene glycol samples are much bigger than the RF coil. This causes the sample to fold around the surface coil under pressure resulting in the samples being damaged. The propagating medium (wooden block) was also not very stable as it was only supported by the sample. To improve the experimental setup, a wooden platform was created to be mounted on the magnet array to provide a level surface for the sample and a sturdier wooden block was created to act as a propagating medium. The platform and its dimensions are shown in Figure 42.

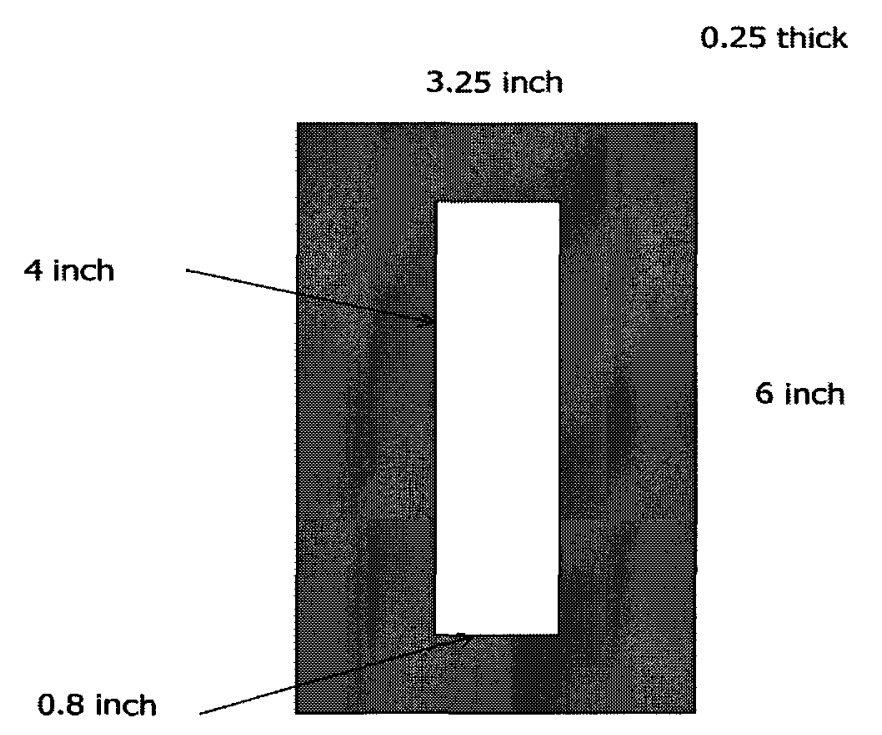

Figure 42 - Wooden Platform that is mounted on top of the magnet array.

The wooden block was created by gluing two blocks on top of each other. The top block has a dimension of $2.1 \times 2.25 \times 1.9$ inch and the bottom block has a dimension of $2.25 \times 3.8 \times 3.8$ inch. Plywood was then attached on the base to lift the block by $1.23 \mathrm{~cm}$ 
allowing a $0.4 \mathrm{~mm}$ compression on the sample. The total height of the block is 4 inches. A slice view of the resulting setup is shown in Figure 43.

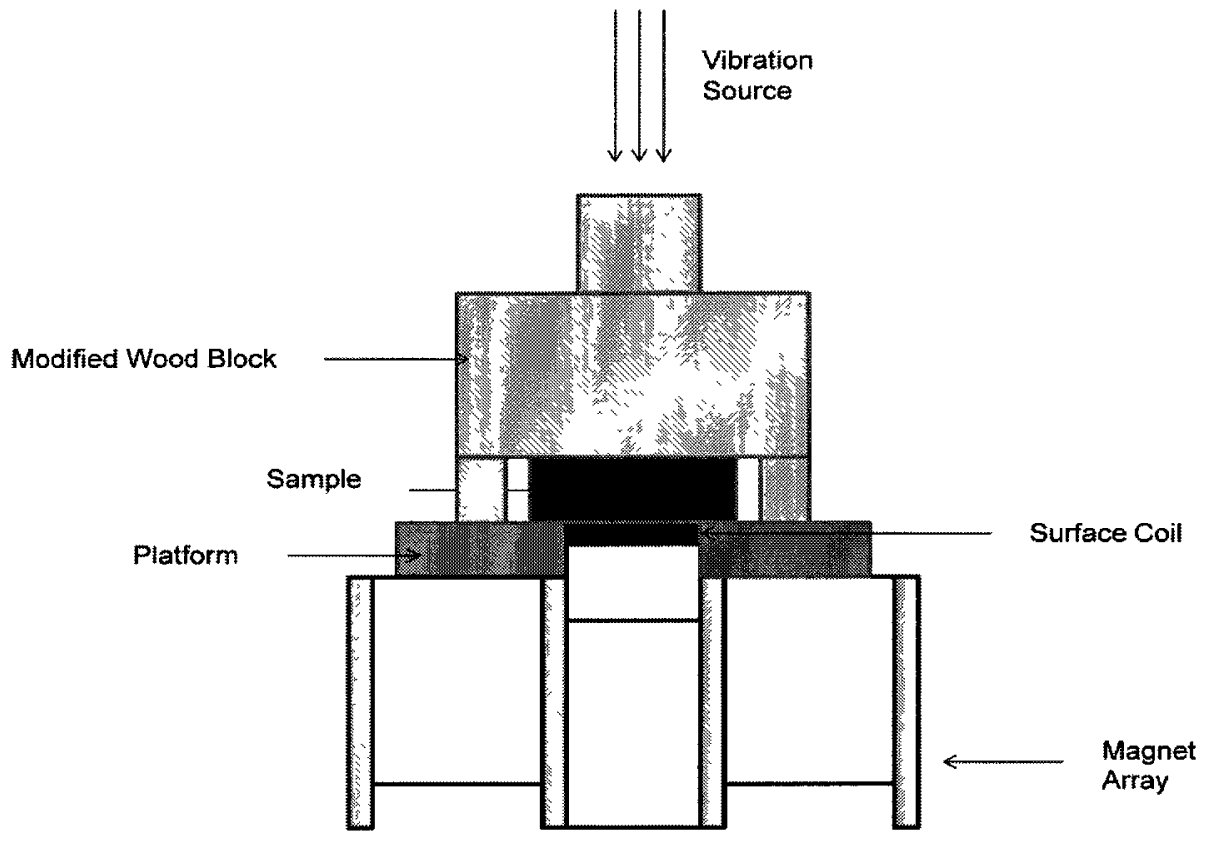

Figure 43 - Slice view of the revised setup.

The Conair massager also was not working very well as it was hard to securely mount the massager on to the position system. To revise this, we used the Dr. Scholl's massager as it could be easily secured on to the positioning system using clamps. The Dr. Scholl's massager also provided stronger vibrations compared to the Conair massager. The modified experimental setup with the Dr. Scholl's massager is shown in Fig. 44. This setup was used for the remaining experiments done for this work. 


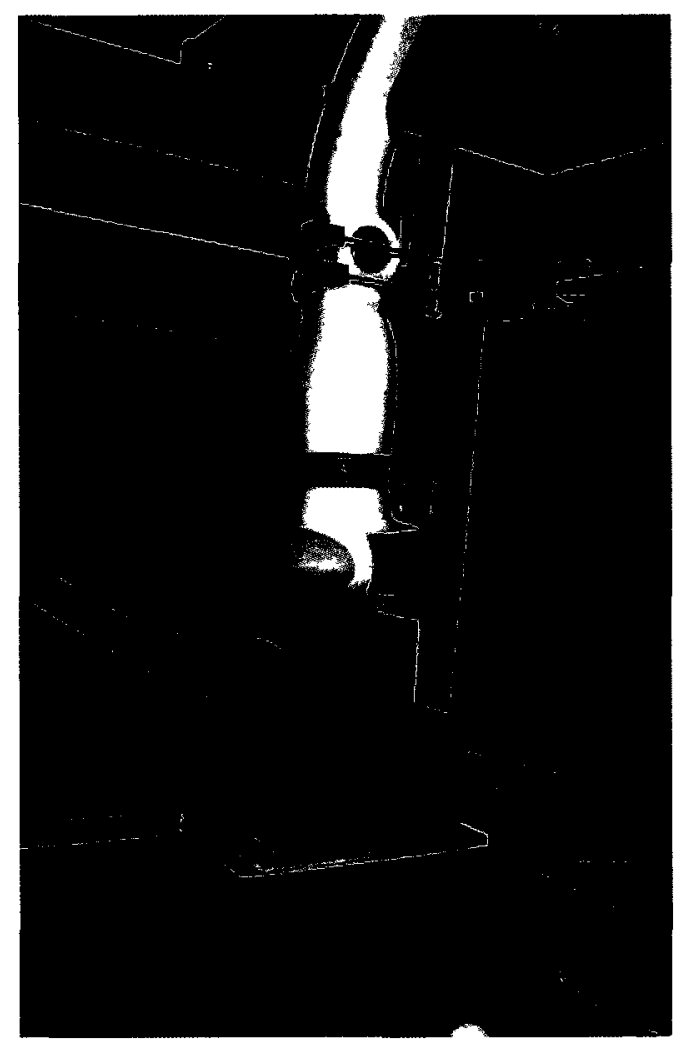

Figure 44 - Experiment setup with Dr. Scholl's Massager.

\subsubsection{P-space Measurements Discussion}

Previous results in Figs. 38-41 show that under no motion, there is no significant signal attenuation whereas under motion, there is significant signal attenuation as $\tau$ increases. The signal attenuation is observed to be decaying linearly in terms of $p$. One noticeable fact from the plots is the unusually high signal intensity when $\tau=0.2 \mathrm{~ms}$ which is equal to $\tau_{\text {train }}$. An experiment was conducted where $\tau$ begins to sample at a time less than $\tau_{\text {train }}$ using the SGSE-CPMG pulse. The resulting $\boldsymbol{p}$-space plot is shown in Fig. 45. 


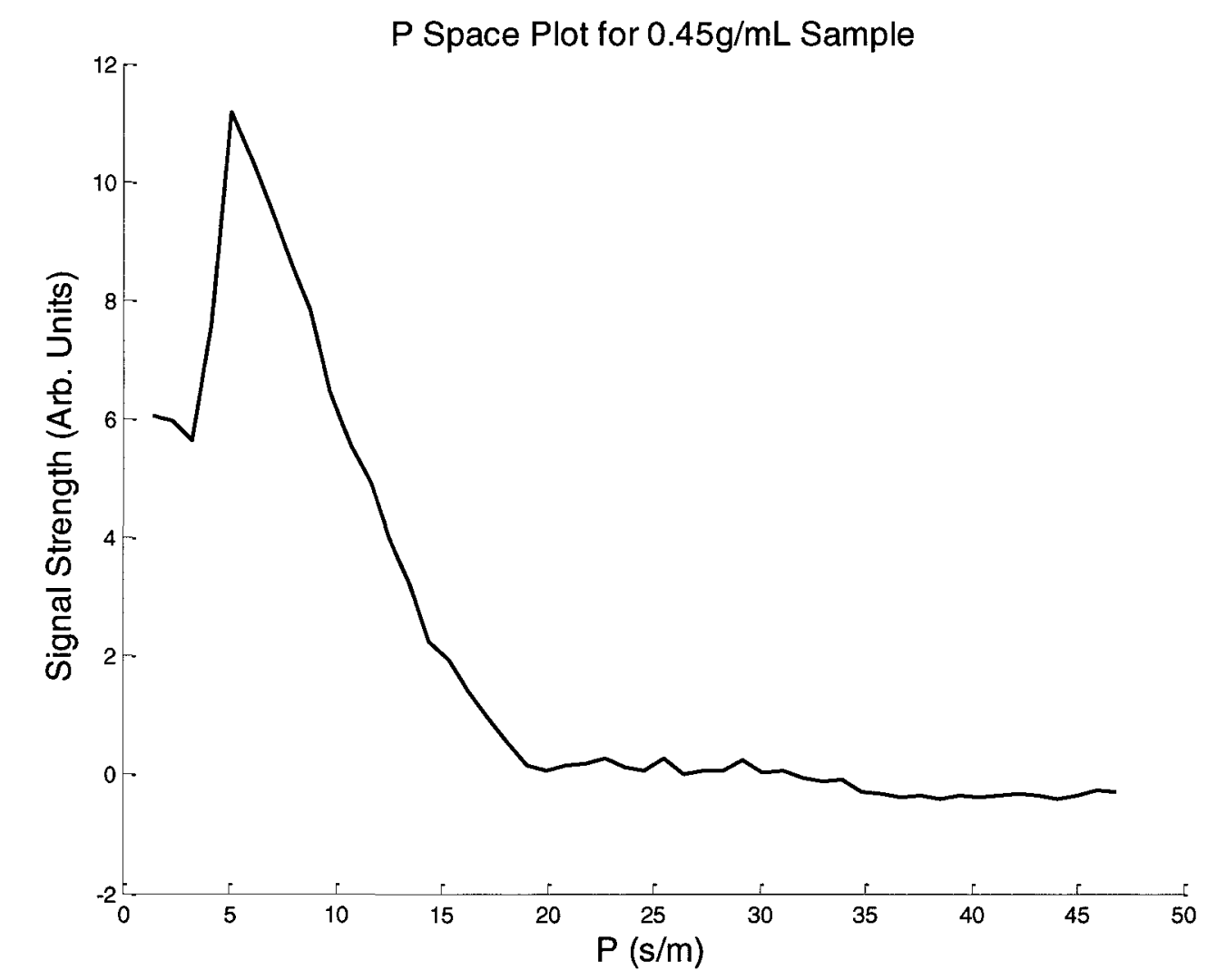

Figure $45-\tau^{2}$ from 0.02 to 2.02 with an increment of $0.04,400$ scans, $0.1 \mathrm{~ms}$ acquisition time, 301 echoes and at 80 data points per echo.

This outcome makes sense because if the velocity encoding period starts at a time less than or equal to $\tau_{\text {train }}$, it is essentially not detecting any motion as previous results show that at $\tau=\tau_{\text {train, }}$ rephrasing occurs fast enough that negligible attenuation is observed. This plot also shows that velocity encoding starts at $\tau$ greater than $0.4 \mathrm{~s}$. This agrees with the data obtained with the silicon rubber using the previous setup in Fig. 37. It is also observed that using the Dr. Scholl's massager, the MRI signal reaches the noise floor at approximately $\boldsymbol{p}=20$ on a stiff sample. Therefore, it is then safe to assume that the MRI signal of samples that are less stiff would attenuate even faster. 


\subsubsection{Ethylene Glycol Gelatin Elasticity Measurements and Results}

The main objective of this thesis was to develop a method to determine the elasticity of samples using a single-sided magnet. A key component of the experiment was to ensure that the force applied by the massager on to the sample was the same for each sample for Eq. 3 to be valid in estimating Young's modulus. To minimize the difference in the force applied to each sample, a set displacement of approximately 0.4 $\mathrm{mm}$ was applied. Even though the force applied on the samples will vary between 0.2$0.6 \mathrm{~N}$ (Fig. 25), the RMS velocity calculated should still be able to determine which samples are stiffer.

The following experiment was performed on the five created ethylene glycol. $\boldsymbol{P}$ space measurement was performed to measure motion. Previous experiments determined that SGSE-CPMG was a faster and more efficient pulse sequence than the CPMG pulse sequence for this particular setup. Therefore, SGSE-CPMG pulse was used for this experiment. It was discussed in section 5.2 .4 .3 that the $0.45 \mathrm{~g} / \mathrm{mL}$ sample MRI signal reaches the noise floor at approximately $p=20$. Furthermore, it was shown that at $\tau$ less than 0.5 , phase refocusing occured fast enough that motion was not encoded. Therefore, $p$-space is sampled at $\tau^{2}=0.06$ to 1.18 at increments of 0.02 or from $\boldsymbol{p}=1.3924$ to 22.7420 at increments of approximately 0.4641 . The rest of the scanning parameters for this experiment were 400 scans, $0.1 \mathrm{~ms} \mathrm{acq,} 601$ echoes, and 80 data points per echo.

The raw MRI signal acquired from the gelatin samples is shown in figure 46 . 
Processing the data as mentioned in section 4.7 results in a $p$-space plot shown in figure 47. Applying the inverse Fourier transform on the data obtained in figure 47 results in a velocity distribution plot shown in figures 48 and 49. Finally, applying Eq 4.2 on the velocity distribution plot, a RMS velocity vs gelatin concentration and RMS velocity vs Young's modulus plots can be obtained shown in figures 50 and 51.
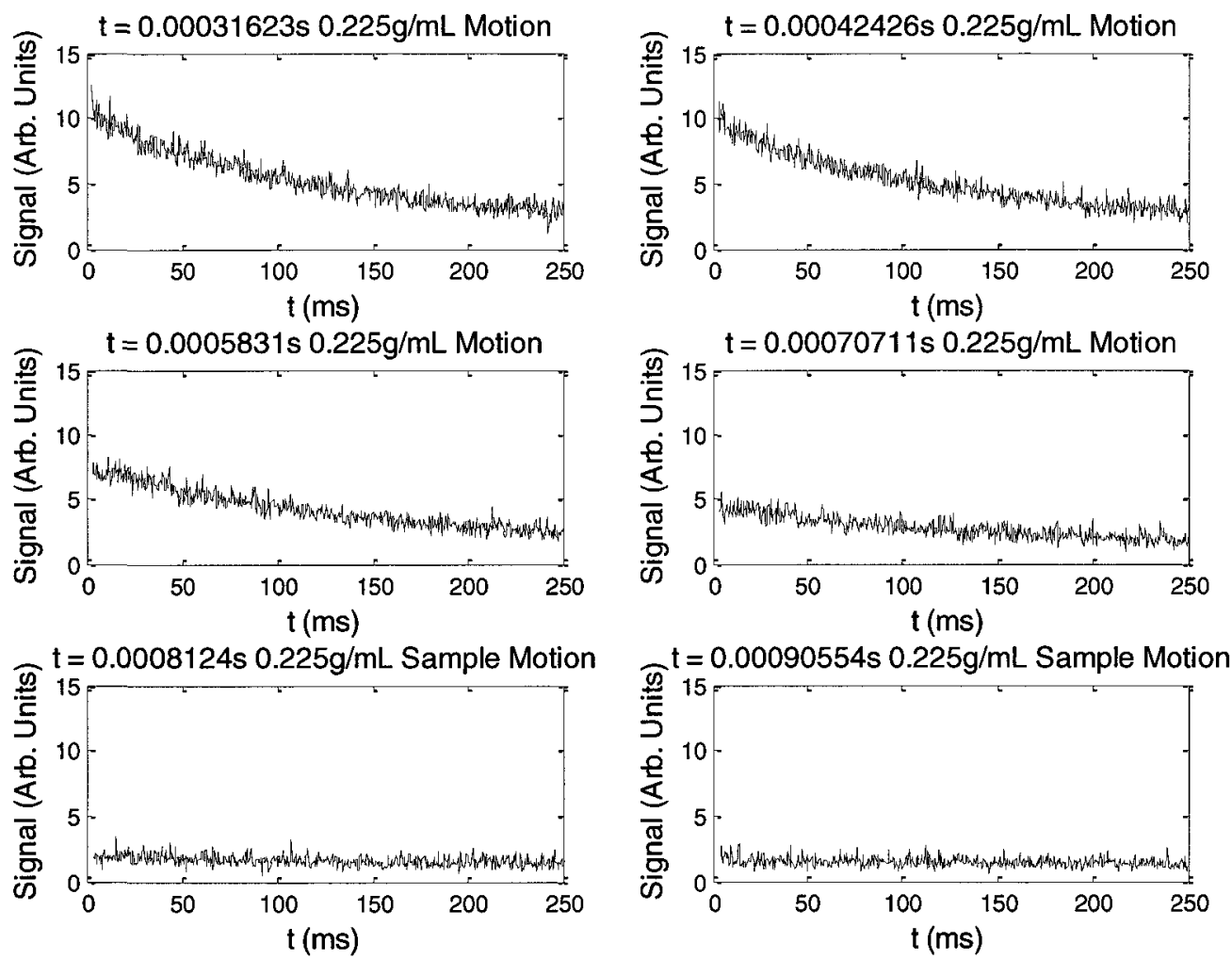

Figure 46 - Echo Peaks of Raw MRI data at various $\tau$ for $0.225 \mathrm{~g} / \mathrm{mL}$ gelatin under motion. 
Signal Intenisty vs Massager Distance from the Surface of the Magnet (Conair Massager)

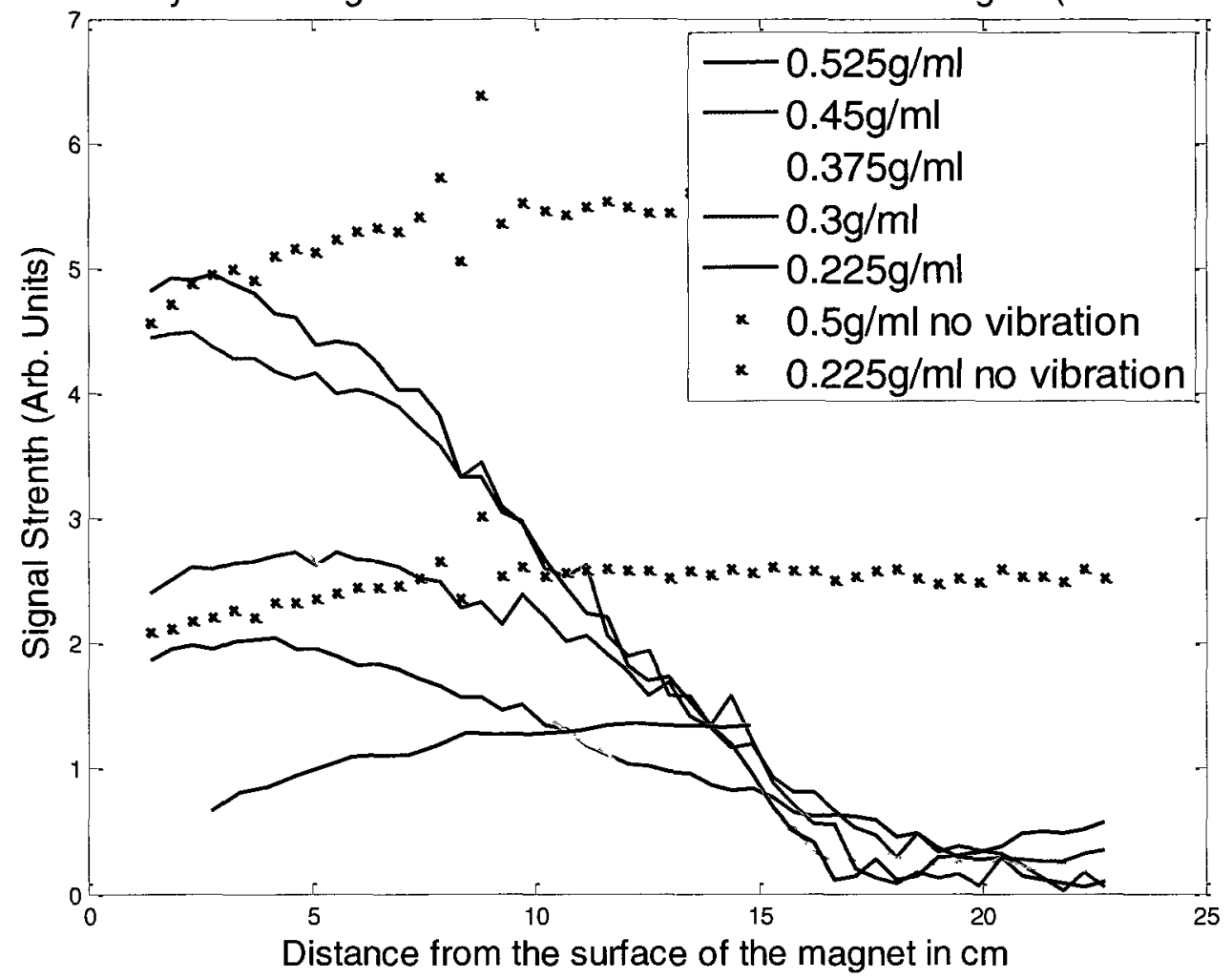

Figure 47 - P-Space plot for all the 5 ethylene glycol gelatin samples under motion.

Two plots are shown with no vibration. 


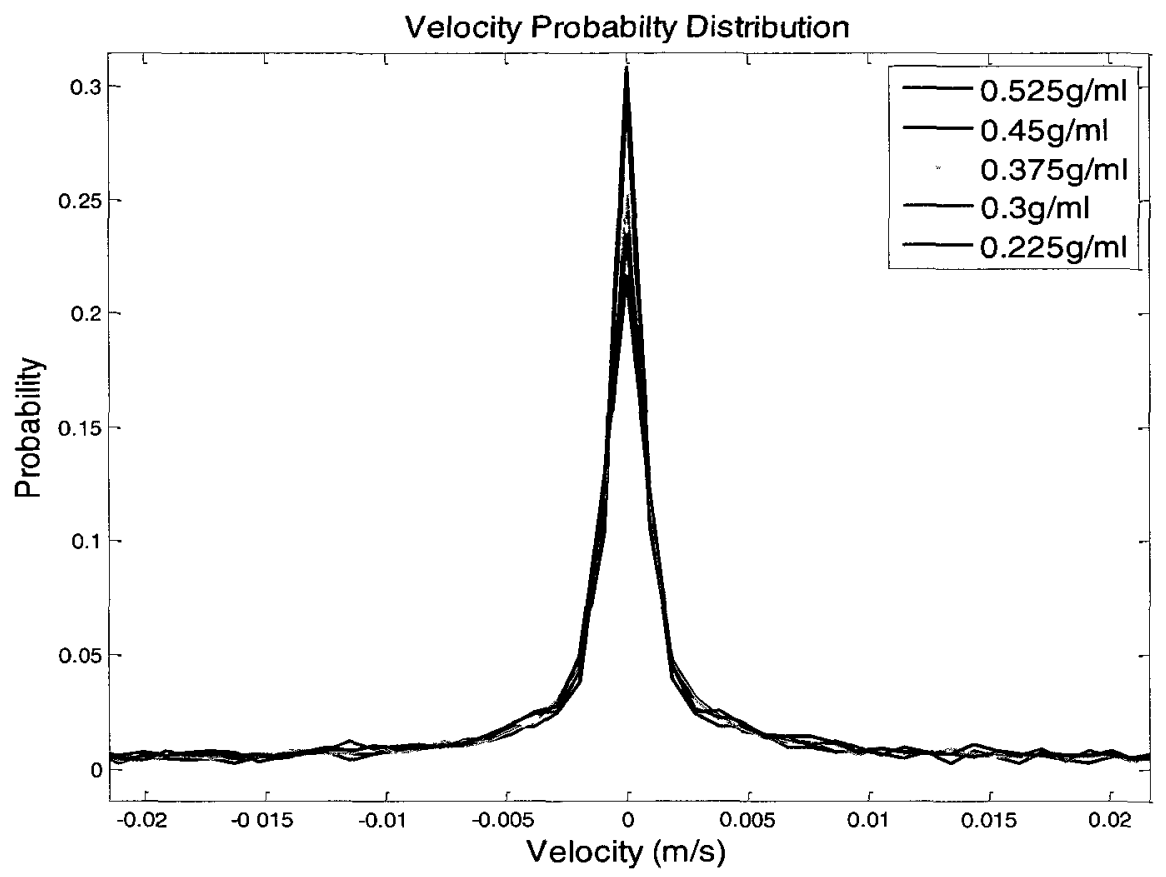

Figure 48 - Velocity Probability Distribution of the five gelatin samples. A close up on the center distribution is shown in Figure 48.

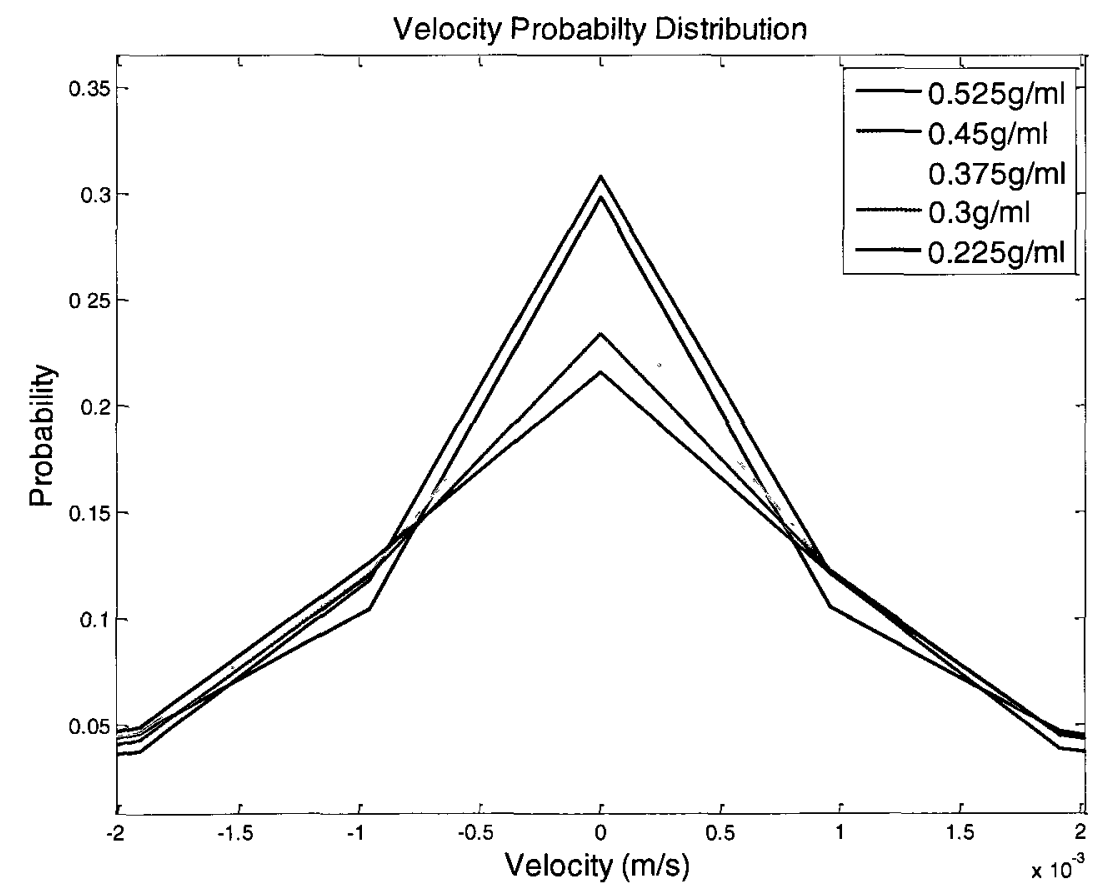

Figure 49 - Velocity Probability Distribution focused at the center. 


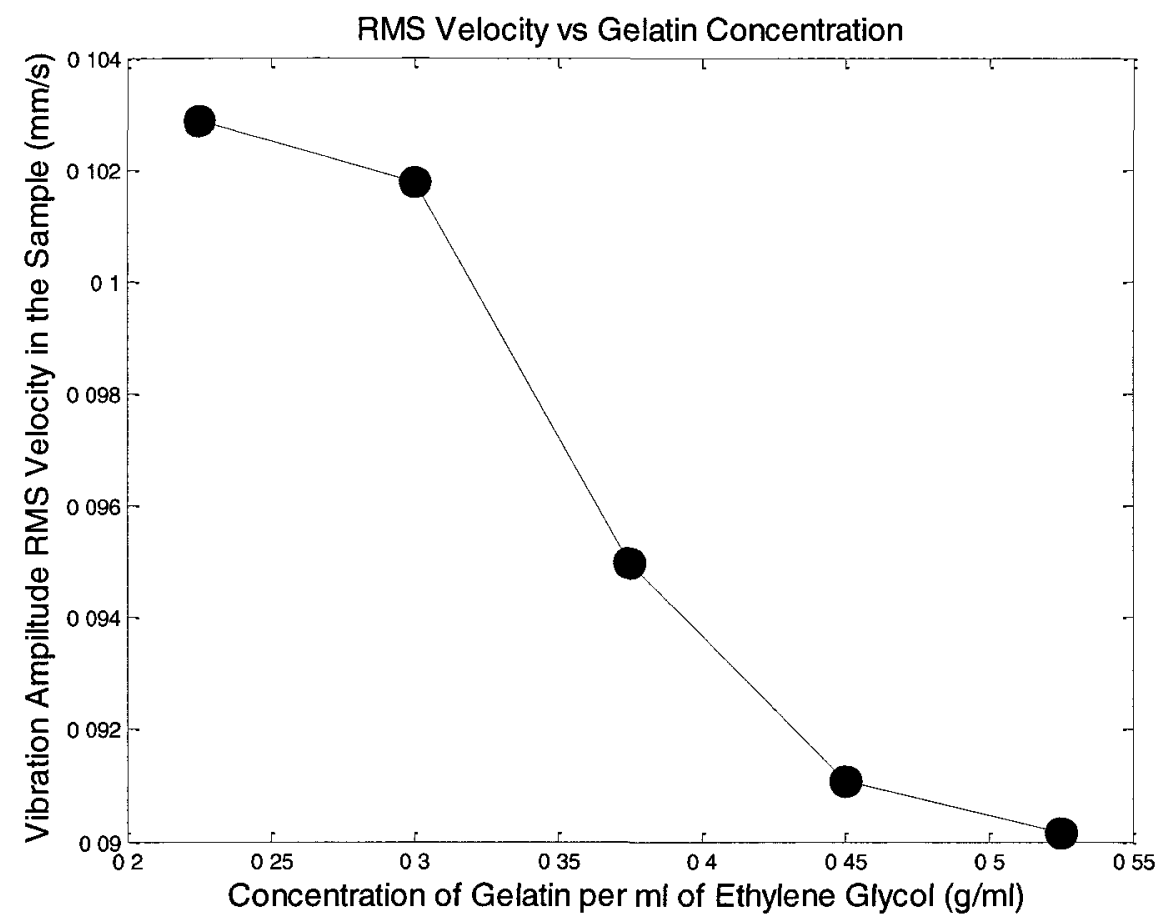

Figure 50 -RMS Velocity vs Gelatin Concentration.

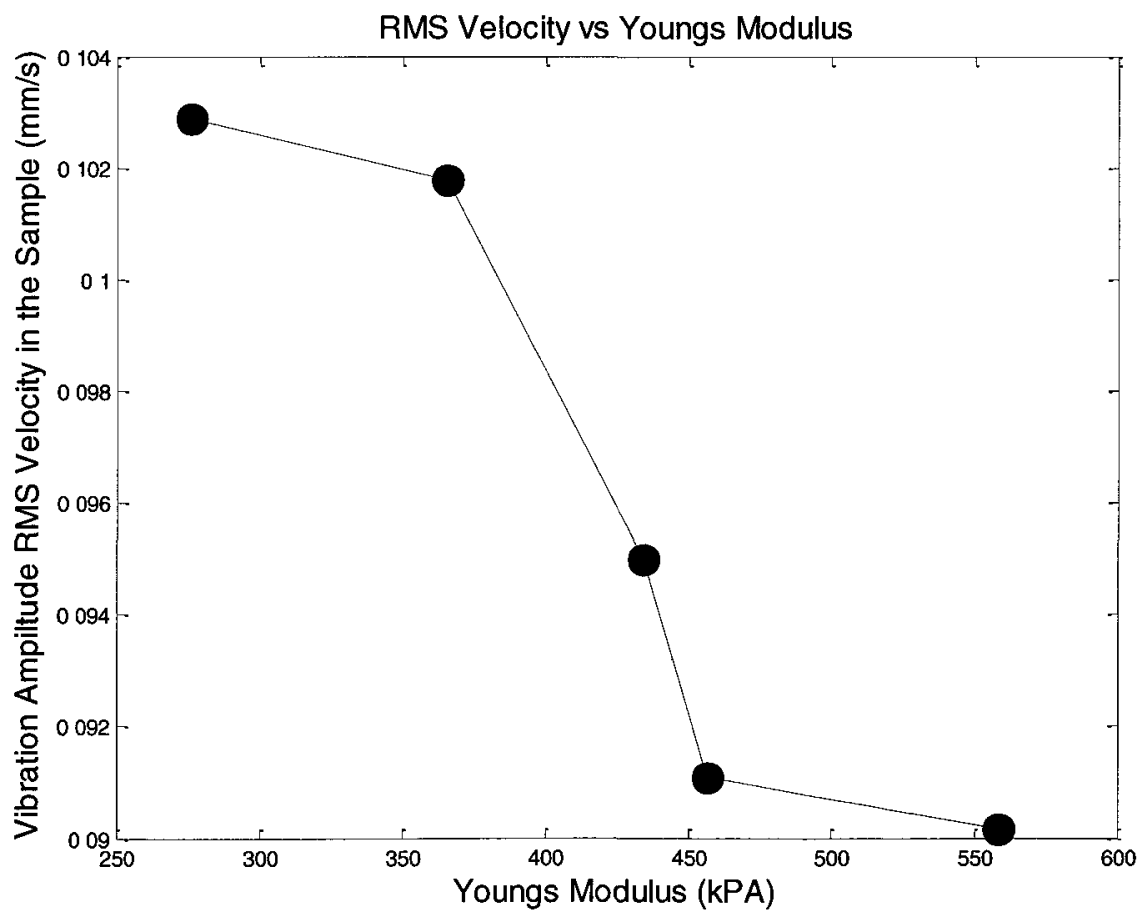

Figure 51 -RMS Velocity vs Estimated Young's modulus. 


\subsubsection{Ethylene Glycol Gelatin Elasticity Discussion}

In Fig. 47, the figure shows the raw MRI signal acquired on a vibrating gelatin sample at various $\tau$. An important observation is that at $\tau$ less than $0.5 \mathrm{~ms}$, no evident signal attenuation occurs in the echoes as mention in previous section. Fig. 48 shows the p-space plot of each sample where each point is obtained by ensemble averaging the echoes at each $\tau$. Under no motion, signal attenuation is not observed. However, magnitude spikes are seen to occur at approximately $p=9 \mathrm{~s} / \mathrm{m}$. This can be due to resonance in the timing of the pulse sequences at that particular instance. Under motion, signal attenuation increases as the Young's modulus decreases. Applying the inverse Fourier transform, a velocity distribution plot is observed shown in Fig. 49 and 50. From this plot, $V_{r m s}$ is then calculated and plotted against gelatin concentration and the measured Young's modulus shown in Fig. 51 and 52 respectively. The final results demonstrate that a stiffer sample has less RMS velocity than a softer sample. This conclusion also stems from the correlation in which the signal attenuation in $p$-space is related to the stiffness of the sample when a constant oscillating force is applied. This is consistent with the theory discussed in section 2.1. However, referring to Eq 2.3, the measured RMS velocity should decay inverse proportionally with increasing Young's Modulus. The results obtained do not reflect this due to many variables. First, the forces applied on the samples were difficult to control. At this point, the best way to control the force applied is to compress the sample as little as possible before the vibration is applied. At small compressions, the force variations between the samples are very small. In addition, a full scan of one sample under the given experimental parameters took 
almost 6 hours ( 400 scans $* 1$ s per scan $* 49$ data points). During this time, the motor of the massager can and will most likely have degraded over the course of the experiment causing the applied frequency and amplitude to vary between samples. The samples can also introduce errors. As the samples were exposed to air, evaporation occurred which may have caused the samples to become stiffer. This was already foreseen and minimized as the chosen solvent, ethylene glycol, already has a boiling point of $197^{\circ}$ compared to water at $100^{\circ}$. Regardless, minimizing scanning time will also minimize the time the sample will be exposed to air. Signal to noise ratio also presented a clear problem as the MRI signal already reached the noise floor at approximately $p=17$ for the softest sample and $p=23$ for the stiffer sample. Increasing the magnetic field strength of the magnet would also increase the signal-to-noise ratio which in turn would allow us to greatly reduce the number of scans needed.

The overall system shows that it is possible to perform MRE using a single-sided magnet. Compared to current elastography modalities such as UE and traditional MRE, the proposed system performed much slower and was only able to obtain elasticity of a homogeneous sample in one direction. However, unlike traditional MRE, the proposed system has potential to analyze elasticity properties of samples not confined in an MRI bore at a much cheaper cost. 


\section{Conclusion and Future Work}

The following chapter concludes the thesis, summarizes the findings and discusses future work.

\subsection{Conclusion}

The overall goal of this research was to develop and implement a portable MR system that can measure elasticity. We have shown a novel way of implementing this system by using a single-sided magnet to generate a magnetic field, and then using velocity imaging techniques to obtain information about Young's modulus of a sample. The developed system showed a strong correlation between MRI signal attenuation and elasticity when an oscillating force was applied on the sample. This relationship in turn provided us with information about the displacement velocity experienced by the sample which is inversely proportional to elasticity. Currently, the clinical feasibility of this system is limited due to the amount of time required to obtain data and the lack of control of the external vibration source. However, with further developments on the system, it can lead to a portable magnetic resonance based elastography system with scan times under 10 minutes, with much greater contrast than conventional MRE systems due to inherent large field gradients of the single-sided magnets and with cost in the range of Ultrasound Elastography systems. 


\subsection{Future Work}

The research presented here was a first step in the direction of designing a portable MRE system for determining tissue elasticity that could eventually be used on humans. Large amounts of work remain to be done to improve the experimental setup and methodology used throughout this thesis before it can be used to perform in-vivo measurements. The following section outlines suggestions for future research.

The single-sided magnet has much room for development. Improving the magnet in terms of the magnetic field strength and the gradient would greatly increase the signalto-noise ratio of the acquired signal and the resolution of the velocity encoding measurements. This will also reduce the number of scans required which will drastically decrease the time required to obtain data.

The largest source of error occurred with the external vibration source. Applying an accurate and repeatable constant oscillating force on the samples is essential to be able to quantitatively calculate Young's modulus. A possible solution in accomplishing this is to place a high precision balance beneath the magnet. Then using a software program, one could simultaneously control the position system and the magnet using a feedback loop to ensure that the oscillating force applied to the system is constant for all the samples. Another possible solution is to obtain a non-magnetic vibrating actuator where frequency and amplitude could be easily controlled. This vibrating actuator needs to produce a strong enough displacement through the gradient. 


\section{Works Cited}

[1] National Cancer Insitute. (2011, January) National Cancer Insitute - Types of Treatment. [Online]. http://www.cancer.gov/cancertopics/treatment/types-of$\underline{\text { treatment }}$

[2] Catherine D. G. Hines, Thorsten A. Bley, Mary J. Lindstrom, and Scott B. Reeder, "Repeatability of Magnetic Resonance Elastography for Quantification of Hepatic Stiffness," Journal of Magnetic Resonance Imaging, vol. 31, no. 3, pp. 725-731, March 2010.

[3] Timothy J. Hall, Mehmet Bilgen, Michael F. Insana, and Thomas A. Krouskop, "Phantom Materials for Elastography," IEEE Trans. Ultrason. Ferroelectr. Freq. Control, vol. 44, no. 6, pp. 1355-1365, November 1997.

[4] Robert R. Edelman, John R. Hesselink, Michael B. Zlatkin, and John V. Crues, Clinical Magnetic Resonance Imaging Volume 1, 3rd ed. Philadelphia, PA: Saunders, 2005.

[5] Malcolm H. Levitt, Spin Dynamics: Basics of Nuclear Magnetic Resonance.: Wiley.

[6] Paul T. Callaghan, Principles of Nuclear Magnetic Resonance Microscopy. Oxford, U.K.: Clarendon, 1991.

[7] R. F. Farr and P. J. Allisy-Roberts, Physics for Medical Imaging. London, Great Britain: W.B. Saunders Company, 1997.

[8] Jerry L. Prince and Jonathan M. Links, Medical Imaging Signals and Systems. NJ, USA: Pearson Prentice Hall, 2006. 
[9] Markus Nilsson and Hannah Rosquist, "q-Space Diffusion MRI: Sequence Development and Phantom Design," Lund University, Lund, Master Thesis 2006.

[10] D.G. Rata, F. Casanova, J. Perlo, D.E. Demco, and B. Blümich, "Self-Diffusion Measurements by a Mobile Single-Sided NMR Sensor with Improved Magnetic Field Gradient," Journal of Magnetic Resonance, vol. 180, no. 2, pp. 229-235, June 2006.

[11] Benedict Newling et al., "Velocity Imaging of Highly Turbulent Gas Flow," Physical Review Letters, vol. 93, no. 15, pp. 154503-(1-4), October 2004.

[12] Joseph Schmitt, "OCT Elastography: Imaging Microscopic Deformation and Strain of Tissue," Optics Express, vol. 3, no. 6, pp. 199-211, September 1998.

[13] Steven G. Adie et al., "Spectroscopic Optical Coherence Elastography," Optics Express, vol. 18, no. 25, pp. 25519-25534, December 2010.

[14] Gyu W. Kim, Byung H. Han, Min H. Cho, and Soo Y. Lee, "X-ray Elastography: A Feasibility Study," in 31st Annual International Conference of the IEEE EMBS, Minneapolis, 2009, pp. 3513 - 3516.

[15] J. Ophir, I. Céspedes, H. Ponnekanti, Y. Yazdi, and X. Li, "Elastography: A Quantitative Method forImaging the Elasticity of BiologicalTissues," Ultrasonic Imaging, vol. 13, no. 2, pp. 111-134, April 1991.

[16] I. Cespedes, J. Ophir, H. Ponnekanti, and N. Maklad, "Elastography: Elasticity Imaging Using Ultrasound with Application to Muscle and Breast In Vivo," Ultrasonic Imaging, vol. 15, no. 2, pp. 73-88, April 1993.

[17] Y. Yamakoshi, J. Sato, and T. Sato, "Ultrasonic imaging of internal vibration of 
soft tissue under forced vibration ," IEEE Trans. Ultrason. Ferroelectr. Freq. Control, vol. 37, no. 2, pp. 45-53, March 1990.

[18] L. Gao, K.J. Parker, and S.K. Alam, "Sonoelasticity Imaging: Theory and Experimental Verification," J. Acoust. Soc. Am., vol. 97, no. 6, pp. 3875-3886, June 1995.

[19] K.J. Parker, L. Gao, S.K. Alam, D. Rubens, and Lerner R.M., "Sonoelasticity Imaging: Theory and Applications," in Ultrasonics Symposium, 1996. Proceedings., 1996 IEEE, San Antonio, 1996, pp. 623-628.

[20] Qing-Li Zhu et al., "Real-Time Ultrasound Elastography: Its Potential Role in Assessment of Breast Lesions ," Ultrasound in Med. \& Biol., vol. 34, no. 8, pp. 1232-1238, August 2008.

[21] Annalisa Berzigotti et al., "Ultrasonographic Evaluation of Liver Surface and Transient Elastography in Clinically Doubtful Cirrhosis," J. Hepatol., vol. 52, no. 6, pp. 846-853, June 2010.

[22] Masahiko Sanada et al., "Clinical Evaluation of Sonoelasticity Measurement in Liver using Ultrasonic Imaging of Internal Forced Low-Frequency Vibration," Ultrasound in Medicine \& Biology, vol. 26, no. 9, pp. 1455-1460, November 2000.

[23] Cédric Schmitt, Anis Hadj Henni, and Guy Cloutier, "Ultrasound Dynamic MicroElastography Applied to the Viscoelastic Characterization of Soft Tissues and Arterial Walls," Ultrasound in Med. \& Biol., vol. 36, no. 10, pp. 1637-1643, October 2010.

[24] R. Muthupillai et al., "Magnetic Resonance Elastography by Direct Visualization 
of Propagating Acoustic Strain Waves," Science, vol. 269, no. 5232, pp. 18541857, September 1995.

[25] Yogesh K. Mariappan, Kevin J. Glaser, and Richard L. Ehman, "Magnetic Resonance Elastography: A Review," Clinical Anatomy, vol. 23, no. 5, pp. 497511, July 2010.

[26] M. Alex Dresner et al., "Magnetic Resonance Elastography of Skeletal muscle," $J$. Magn. Reson. Imaging, vol. 13, no. 2, pp. 269-276, February 2001.

[27] Ingolf Sack, Johannes Bernarding, and Jürgen Braun, "Analysis of Wave Patterns in MR Elastography of Skeletal Muscle Using Coupled Harmonic Oscillator Simulations," Magnetic Resonance Imaging, vol. 20, no. 1, pp. 95-104, January 2002.

[28] Sebastian Papazoglou, Jens Rump, Jürgen Braun, and Ingolf Sack, "Shear Wave Group Velocity Inversion in MR Elastography of Human Skeletal Muscle," Magnetic Resonance in Medicine, vol. 56, no. 3, pp. 489-497, September 2006.

[29] Dieter Klatt, Sebastian Papazoglou, Jürgen Braun, and Ingolf Sack, "Viscoelasticity-Based MR Elastography of Skeletal Muscle," Physics in Medicine and Biology, vol. 55, no. 21, pp. 6445-6459, January 2010.

[30] Benjamin Robert, Ralph Sinkus, Jean-luc Gennisson, and Mathias Fink, "Application of DENSE-MR-Elastrography to the Human Heart," Magnetic Resonance in Medicine, vol. 62, no. 5, pp. 1155-1163, November 2009.

[31] Arunark Kolipaka et al., "MR Elastography as a Method for the Assessment of Myocardial Stiffness: Comparison with an Established Pressure-Volume Model in 
a Left Ventricular Model of the Heart," Magnetic Resonance in Medicine, vol. 62, no. 1, pp. 135-140, Jully 2009.

[32] Arunark Kolipaka et al., "Evaluation of a Rapid, Multiphase MRE Sequence in a Heart-Simulating Phantom," Magnetic Resonance in Medicine, vol. 62, no. 3, pp. 691-698, September 2009.

[33] Arunark Kolipaka, Philip A. Araoz, Kiaran P. McGee, Armando Manduca, and Richard L. Ehman, "Magnetic Resonance Elastography as a Method for the Assessment of Effective Myocardial Stiffniess Throughout the Cardiac Cycle," Magnetic Resonance in Medicine, vol. 64, no. 3, pp. 862-870, September 2010.

[34] Meng Yin et al., "Assessment of Hepatic Fibrosis With Magnetic Resonance Elastography," Clin. Gastroenterol. Hepatol., vol. 5, no. 10, pp. 1207-1213, October 2007.

[35] Patrick Asbach et al., "Assessment of Liver Viscoelasticity Using Multifrequency MR Elastography," Magn. Reson. Med., vol. 60, no. 2, pp. 373-379, August 2008.

[36] D. A. Herzka, M. S. Kotys, R. Sinkus, R. I. Pettigrew, and A. M. Gharib, "Manetic Resonance Elastography in the Liver at 3 Tesla Using a Second Harmonic Approach," Magnetic Resoance in Medicine, vol. 62, no. 2, pp. 284-291, August 2009.

[37] Sabine F. Bensamoun et al., "Measurement of Liver Stiffness With Two Imaging Techniques: Magnetic Resonance Elastography and Ultrasound Elastometry," Journal of Magnetic Resonance Imaging, vol. 28, no. 5, pp. 12887-1292, November 2008. 
[38] B. C. Goss, K. P McGee, E. C. Ehman, A. Manduca, and R. L. Ehman, "Magnetic Resonance Elastography of the Lung: Technical Feasibility," Magnetic Resonance in Medicine, vol. 56, no. 5, pp. 1060-1066, November 2006.

[39] Kiaran P. McGee, Rolf D. Hubmayr, and R. L. Ehman, "MR Elastography of the Lung With Hyperpolarized 3He," Magnetic Resonance in Medicine, vol. 59, no. 1, pp. 14-18, January 2008.

[40] L. Xu et al., "Magnetic Resonance Elastography of Brain Tumors: Preliminary Results," Acta Radiol., vol. 48, no. 1, pp. 112-115, February 2007.

[41] A. J. Pattison et al., "Time-Harmonic Magnetic Resonance Elastography of the Normal Feline Brain," Journal of Biomechanics, vol. 43, no. 14, pp. 2747-2752, October 2010.

[42] R. Sinkus et al., "High-Resolution Tensor MR Elastography for Breast Tumour Detection," Phys. Med. Biol., vol. 45, no. 6, pp. 1649-1664, June 2000.

[43] Ralph Sinkus et al., "MR Elastography of Breast Lesions: Understanding the Solid/Liquid Duality can Improve the Specificity of Contrast-Enhanced MR Mammography," Magnetic Resonance in Medicine, vol. 58, no. 6, pp. 1135-1144, December 2007.

[44] Donald B. Plewes, Jonathan Bishop, Abbas Samani, and Justin Sciarretta, "Visualization and Quantification of Breast Cancer Biomechanical Properties with Magnetic Resonance Elastography," Physics in Medicine and Biology, vol. 45, no. 6, pp. 1591-1610, June 2000 .

[45] P. R. Perrinez, F. E. Kennedy, E. E. W. Van Houten, J. B. Weaver, and K. D. 
Paulsen, "Modeling of Soft Poroelastic Tissue in Time-Harmonic MR Elastography," IEEE Transactions on Biomedical Engineering, vol. 56, no. 3, pp. 598-608, Match 2009.

[46] Z.T.H. Tse et al., "Magnetic Resonance Elastography Hardware Design: A Survey," Proc. Inst. Mech. Eng. H., vol. 223, no. 4, pp. 497-514, May 2009.

[47] Juergen Braun, Karl Braun, and Ingolf Sack, "Electromagnetic Actuator for Generating Variably Oriented Shear Waves in MR Elastography," Magn. Reson. Med., vol. 50, no. 1, pp. 220-222, July 2003.

[48] Shadi F. Othman, Huihui Xu, Thomas J. Royston, and Richard L. Magin, "Microscopic Magnetic Resonance Elastography ( $\mathrm{MMRE),"} \mathrm{Magn.} \mathrm{Reson.} \mathrm{Med.,}$ vol. 54, no. 3, pp. 605-615, September 2005.

[49] Jun Chen, Cheng Ni, and Tiange Zhuang, "Imaging Mechanical Shear Waves Induced by Piezoelectric Ceramics in Magnetic Resonance Elastography," Chin. Sci. Bull., vol. 51, no. 6, pp. 755-760, March 2006.

[50] Tao Wu, Joel P. Felmlee, James F. Greenleaf, Stephen J. Riederer, and Richard L. Ehman, "MR Imaging of Shear Waves Generated by Focused Ultrasound," Magn. Reson. Med., vol. 43, no. 1, pp. 111-115, January 2000.

[51] Kathryn R. Nightingale, Mark L. Palmeri, Roger W. Nightingale, and Gregg E. Trahey, "On the Feasibility of Remote Palpation Using Acoustic Radiation Force," J. Acoust. Soc. Am., vol. 110, no. 1, pp. 625-634, July 2001.

[52] J. Bercoff, M. Tanter, and M. Fink, "Supersonic Shear Imaging: A New Technique for Soft Tissue Elasticity Mapping," IEEE Trans. Ultrason. Ferroelectr. Freq. 
Control, vol. 51, no. 4, pp. 396-409, April 2004.

[53] Rémi Souchon et al., "Transient MR Elastography (t-MRE) Using Ultrasound Radiation Force: Theory, Safety, and Initial Experiments In Vitro," Magn. Reson. Med., vol. 60, no. 4, pp. 871-881, October 2008.

[54] M. Radicke et al., "Acoustic Radiation Contrast in MR Images for Breast Cancer Diagnostics - Initial Phantom Study," Ultrasound in Med. \& Biol., vol. 37, no. 2, pp. 253-261, February 2011.

[55] A. Manduca et al., "Magnetic Resonance Elastography: Non-Invasive Mapping of Tissue Elasticity," Med. Image Anal., vol. 5, no. 4, pp. 237-254, December 2001.

[56] Mikio Suga, Toshiyuki Aga, and Kotaro Minato, "Development of a Magnetic Resonance Elastic Microscope System," in Proceedings of the 26th Annual International Conference of the IEEE EMBS, San Francisco, 2004, pp. 1025-1027.

[57] Raja Muthupillai et al., "Magnetic Resonance Imaging of Transverse Acoustic Strain Waves," Magn. Reson. Med., vol. 36, no. 2, pp. 266-274, August 1996.

[58] John Rydberg et al., "Fast Spin-Echo Magnetic Resonance Elastography of the Brain," in International Society for Magnetic Resonance in Medicine, Glasgow, 2001, p. 1647.

[59] O. Bieri, S. Maderwald, M.E. Ladd, and K. Scheffler, "Balanced Alternating Steady-State Elastography," Magn. Reson. Med., vol. 55, no. 2, pp. 223-241, February 2006.

[60] Stefan Maderwald, Kai Uffmann, Craig. J. Galbán, Armin De Greiff, and Mark E. Ladd, "Accelerating MR Elastography: A Multiecho Phase-Contrast Gradient-Echo 
Sequence," J. Magn. Reson. Imaging, vol. 23, no. 5, pp. 774-780, May 20062006.

[61] Jens Rump, Dieter Klatt, Jürgen Braun, Carsten Warmuth, and Ingolf Sack, "Fractional Encoding of Harmonic Motions in MR Elastography," Magnetic Resonance in Medicine, vol. 57, no. 2, pp. 388-395, February 2007.

[62] Jennifer Oudry et al., "Cross-Validation of Magnetic Resonance Elastography and Ultrasound-Based Transient Elastography: A Preliminary Phantom Study," Journal of Magnetic Resonance Imaging, vol. 30, no. 5, pp. 1145-1150, November 2009.

[63] Vinayak Dutt et al., "Acoustic Shear-Wave Imaging Using Echo Ultrasound Compared to Magnetic Resonance Elastography," Ultrasound in Medicine \& Biology, vol. 26, no. 3, pp. 397-403, March 2000.

[64] Pablo J. Prado, Bernhard Blümich, and Udo Schmitz, "One-Dimensional Imaging with a Palm-Size Probe," J. Magn. Reson., vol. 144, no. 2, pp. 200-206, June 2000.

[65] J. Perlo, F. Casanova, and B. Blümich, "Profiles with Microscopic Resolution by Single-Sided NMR," J. Magn. Reson., vol. 176, no. 1, pp. 64-70, September 2005.

[66] F. Cassanova, J. Perlo, and B. Blümich, "Velocity Distributions Remotely Measured with a Single-Sided NMR Sensor," Journal of Magnetic Resonance, vol. 171, no. 1, pp. 124-130, November 2004.

[67] F. Casanova and B. Blümich, "Two-Dimensional Imaging with a Single-Sided NMR Probe," J. Magn. Reson., vol. 163, no. 1, pp. 38-45, July 2003.

[68] J. Perlo, F. Casanova, and B. Blümich, "3D Imaging with a Single-Sided Sensor: An Open Tomograph," J. Magn. Reson., vol. 166, no. 2, pp. 229-235, February 2004. 
[69] A.E. Marble, I.V. Mastikhin, B.G. Colpitts, and B.J. Balcom, "An Analytical Methodology for Magnetic Field Control in Unilateral NMR," J. Magn. Reson., vol. 174, no. 1, pp. 78-87, May 2005.

[70] Andrew E. Marble, Igor V. Mastikhin, Bruce G. Colpitts, and Bruce J. Balcom, "A Compact Permanent Magnet Array with a Remote Homogeneous Field," Journal of Magnetic Resonance, vol. 186, no. 1, pp. 100-104, May 2007.

[71] Andrew E. Marble, Igor V. Mastikhin, Bruce G. Colpitts, and Bruce J. Balcom, "A Constant Gradient Unilateral Magnet for Near-Surface MRI Profiling," Journal of Magnetic Resonance, vol. 183, no. 2, pp. 228-234, December 2006.

[72] Emil Veliyulun, Igor V. Mastikhin, Andrew E. Marble, and Bruce J. Balcom, "Rapid Determination of the Fat Content in Packaged Dairy Products by Unilateral NMR," J. Sci. Food Agric., vol. 88, no. 14, pp. 2563-2567, November 2008.

[73] Bruker Optics. (2011, January) The Minispec TD-NMR Bruker Otpics. [Online]. http://www.brukeroptics.com/minispec.html? \&print=1\%C3\%82\%C2\%B0\%3FL $\%$ $\underline{3 \mathrm{D} 0}$

[74] William G. Bradley. (2011, March) FUNDAMENTALS OF MRI: Part III. [Online]. http://www.e-radiography.net $/ \mathrm{mrict} /$ fund $\% 20 \mathrm{mr} 3 /$ fund $\% 20 \mathrm{mr} 3$. htm

[75] Velmex INC. (2011, February) BISLIDE Manual and Motor Driven Positioning System. [Online]. http://www.bislide.com/

[76] M.D. Hürlimann, "Optimization of Timing in the Carr-Purcell-Meiboom-Gill Sequence," Magn. Reson. Imaging, vol. 19, no. 3, pp. 375-378, April 2001.

[77] Martin O. Culjat, David Goldenberg, Priyamvada Tewari, and Rahul S. Singh, "A 
Review of Tissue Substitutes for Ultrasound Imaging," Ultrasound in Med \& Biol., vol. 36, no. 6, pp. 861-873, June 2010.

[78] Toshio Kondo and Mituyoshi Kitatuji, "New Tissue Mimicking Materials for Ultrasound Phantoms," in 2005 IEEE Ultrasonics Symposium, Rotterdam, 2005, pp. 1664-1667.

[79] Wikipedia. (2011, January) Simple linear regression. [Online]. http://en.wikipedia.org/wiki/Simple linear_regression 